JOURNAL OF THE

AMERICAN MATHEMATICAL SOCIETY

Volume 9, Number 1, January 1996

\title{
ZARISKI GEOMETRIES
}

\author{
EHUD HRUSHOVSKI AND BORIS ZILBER
}

\section{INTRODUCTION}

Let $k$ be an algebraically closed field. The set of ordered $n$-tuples from $k$ is viewed as an $n$-dimensional space; a subset described by the vanishing of a polynomial, or a family of polynomials, is called an algebraic set, or a Zariski closed set. Algebraic geometry describes the behavior of these sets. The goal of this paper is a converse. We start with a set $X$, together with a collection of subsets of the powers of $X$, satisfying certain axioms of a geometric nature. We then show that the points of $X$ can be identified with a curve over an algebraically closed field $k$, in such a way that the given collection of sets is precisely the family of Zariski closed ones.

This project is of course familiar if one allows only subsets of $k^{n}$ defined by linear equations. The reader is referred to E. Artin's Geometric algebra, Introduction to Chapter II [AE], paraphrased above, which could serve as a better introduction to the present paper.

As customary we use topological language to describe our axioms for the algebraic sets. We recall some pertinent terms (see e.g. [Ha]). A topological space is Noetherian if it has the descending chain condition on closed subsets. A closed set is irreducible if it is not the union of two proper closed subsets. If $X$ is Noetherian, then every closed set can be written as a finite union of irreducible closed sets. These are uniquely determined (provided no one is a subset of the other), and are called the irreducible components of the given set. We say that $X$ has dimension $n$ if $n$ is the maximal length of a chain of closed irreducible sets $C_{0} \subset C_{2} \subset \cdots \subset C_{n}$ (proper inclusions). The dimension of a closed set is its dimension as a subspace of $X$. A map $f$ is closed if the image of a closed set is closed. If $X$ is irreducible and a property $P$ holds for all elements of $X$ outside of a proper closed subset, we say that $P$ holds generically on $X$, or that a generic point satisfies $P$. If $C \subseteq E \times Y$ and $a \in E$, we let $C(a)=\{y \in Y:(a, y) \in C\}$.

Definition. A Zariski geometry on a set $X$ is a topology on $X^{n}$ for each $n$, satisfying:

(Z0) Let $f_{i}$ be a constant $\operatorname{map}\left(f_{i}\left(x_{1}, \ldots, x_{n}\right)=c\right)$ or a projection $\left(f_{i}\left(x_{1}, \ldots, x_{n}\right)=x_{j(i)}\right)$. Let $f(x)=\left(f_{1}(x), \ldots, f_{m}(x)\right)$. Then $f: X^{n} \rightarrow X^{m}$ is continuous. The diagonals $x_{i}=x_{j}$ of $X^{n}$ are closed.

Received by the editors November 11, 1992 and, in revised form, September 1, 1993

1991 Mathematics Subject Classification. Primary 03C68, 14A99; Secondary 03C65, 14 E10.

The first author was supported by National Science Foundation grant 9106711-DMS. 
(Z1) Let $C$ be a closed irreducible subset of $X^{n}$, and let $\pi$ be the projection to $X^{k}$. Then there exists a proper closed subset $F$ of $\operatorname{cl}(\pi C)$ such that $\pi C \supseteq \operatorname{cl}(\pi C)-F$.

(Z2) $X$ is irreducible and uniformly one-dimensional: if $C \subseteq X^{n} \times X$ is closed, then for some $m$, for all $a \in X^{n}, C(a)=X$ or $|C(a)| \leq m$.

(Z3) (Dimension theorem) $\operatorname{dim}\left(X^{n}\right) \leq n$. Let $U$ be a closed irreducible subset of $X^{n}$, and let $T_{i j}$ be the diagonal $x_{i}=x_{j}$. Then every component of $U \cap T_{i j}$ has dimension $\geq \operatorname{dim}(U)-1$.

Comments. (Z0) is needed in order to relate the various topologies; it replaces the classical understanding that the topology on $X^{n}$ is the product topology.

$X$ is called complete if all projection maps are closed. Note that (Z1) then follows trivially. We prefer not to assume completeness axiomatically, since we wish not to exclude affine models. From a model theoretic point of view (Z1) is just "quantifier elimination"; this will be explained in $\S 2$.

(Z2) states that $X$ is one dimensional, i.e., every proper closed set is finite, but in a way uniform for families of closed subsets.

(Z3) is the key structural condition. It plays the dual role of giving a basic dimension theory for closed sets, and of allowing later a theory of specializations, leading up to what is implicitly a construction of a tangent space. (This suggests that (Z3) can be viewed as a smoothness condition on $X$; see the remark following 1.1 below.)

The axioms we have so far are valid also in the linear situation, where $k$ is identified with a field $k$, and a closed subset of $k^{n}$ is one defined by linear equations. In the linear case, $k$ need not be algebraically closed or even commutative; we refer to the resulting structure as the linear Zariski geometry over $k$. To rule out this class we assert the existence of a rich enough family of plane curves.

By a plane curve over $X$ we mean an irreducible one-dimensional subset of $X^{2}$. A family of plane curves consists of a closed irreducible set $E \subseteq X^{n}$ (parametrizing the family), and a closed irreducible $C \subseteq E \times X^{2}$, such that $C(e)$ is a plane curve for generic $e \in E$.

Definition. A Zariski geometry $X$ is very ample if there is a family $C \subseteq E \times X^{2}$ of plane curves such that:

(i) For generic $(a, b) \in X^{2}$ there exists a curve $C(e)$ passing through $a, b$.

(ii) For any $a, b \in X^{2}$ there exists $e \in E$ such that $C(e)$ passes through just one of $a, b$.

If only (i) holds, $X$ is called ample.

Any smooth algebraic curve $C$ can be viewed as a Zariski geometry. One takes the closed subsets to be the Zariski closed subsets of $C^{n}$ for each $n$. Our main result is the converse statement.

Theorem A. Let $X$ be a very ample Zariski geometry. Then there exists a smooth curve $C$ over an algebraically closed field $F$, such that $X, C$ are isomorphic as Zariski geometries.

Here $C$ is not necessarily complete (but is complete in the sense of algebraic geometry iff $X$ is complete as a Zariski geometry). It is worth noting the following complement, showing that $C, F$ are unique. 
Proposition 1.1. Let $C$ be a smooth curve over an algebraically closed field $F$, and $C^{\prime}$ be a smooth curve over a field $F^{\prime}$. Suppose $h: C \rightarrow C^{\prime}$ is an isomorphism of Zariski geometries (the induced map on $C^{n}$ is a homeomorphism for each $n$ ). Then there exists an isomorphism of fields $h_{F}: F \rightarrow F^{\prime}$. If $F, F^{\prime}$ are identified via $h_{F}$, then $h$ becomes an isomorphism of algebraic varieties.

Remark. Let $C$ be a curve, and suppose that the collection of Zariski closed subsets of $C^{n}$ makes it a Zariski geometry. Then $C$ is nonsingular, or at worst has only cusps for singularities, in the sense that the map $f: \widetilde{C} \rightarrow C$ from the normalization of $C$ to $C$ is bijective. This follows from 4.7, 4.8 to be proved below. (Resolution of cusps is irrelevant from the point of view of the Zariski structure; it is natural to consider only curves for which bijective coverings are isomorphisms, and then the Zariski condition (Z3) implies normality, hence smoothness.)

An arbitrary Zariski geometry can also be closely analyzed. If $X$ is ample, but not very ample, it can be shown to be in a certain sense a finite cover of the projective line over an algebraically closed field (Theorem B). However no analog of the Riemann existence theorem is valid here; there exist finite covers of the projective line which do not arise from algebraic curves (Theorem $\mathrm{C}$ ).

If $X$ is not ample, but in a certain technical sense nondegenerate, then there exists a division ring $k$ canonically associated with $X$, and $X$ is closely related to the linear Zariski geometry over $K$. One shows that $X$ interprets an Abelian group $A$ ([Hr2]), and that the constructible subsets of $A^{n}$ are Boolean combinations of cosets ([HP]). A precise structure theorem is then obtained for $A$ ([HL]). (This paper uses the constructible category, described in $\S 2$, but the result can be converted to the Zariski framework, since one can identify the closed sets among the constructible ones: they are the finite unions of cosets of constructible subgroups of $A^{n}$.)

$X$ is called degenerate if the only nonconstant families of plane curves consist of curves $\{a\} \times X$ and $X \times\{a\}$; the degenerate Zariski geometries have not been studied in detail. In this paper we restrict ourselves to the ample case.

One can also define Zariski geometries of dimension higher than one (see 3.10). Then any compact complex analytic manifold can be viewed as a Zariski geometry, the closed sets being the closed analytic subvarieties. The theorems proved here are geared for such higher-dimensional applications. (This is part of the reason for insisting on removing the completeness assumption.) We refer the reader to [HZ] for some statements of this type. In higher dimensions, one may have degenerate, ample, and nonample parts of the same geometry; they are not in general easily separable. Here we will consider higher-dimensional objects only insofar as they arise from, and are needed for the analysis of, one-dimensional ones. (Model theoretically, these are called "almost strongly minimal"; they are characterized by the existence of enough multi-valued maps into a one-dimensional geometry to separate points. This holds if the points of the geometry are separated by a family of irreducible closed sets of codimension one.)

A partial analog to Theorem 1 in higher dimensions follows immediately from our results; if $X$ is an almost strongly minimal Zariski geometry of dimension 2 or more, and there exists a family of curves on $X$ such that any two points are separated by a curve and any two points lie jointly on some curve, then there exists a dense open subset of $X$ isomorphic to an algebraic variety. It is likely that $X$ arises globally from an algebraic space in the sense of M. Artin, but we have not proved this. 
We will consistently use both the topological and the constructible category. The following section, $\S 2$, contains a second introduction, from the latter point of view. Results more easily stated in the constructible language are stated there. The structure of the rest of the paper is as follows.

$\S 2$. Following the introduction, it is shown that the Zariski axioms have the expected implications in the constructible category. The reader who approaches the paper with model theoretic applications in mind may be willing to take these for granted.

$\S 3$. Model theoretic preliminaries.

$\S 4$. Theory of specializations. In 4.1 it is shown that our assumptions carry over to elementary extensions; in most applications this is known in advance, and so may again be skipped. The second part of the section on the other hand develops the central limit process that we will use to "differentiate" the given structure and recognize underlying group structures.

$\S 5$. Imaginary elements; manifolds. We introduce manifolds over a Zariski geometry. This will give a topological structure to the field, which will be found by constructible methods.

$\S 6$. Interpreting the field. We work in the constructible framework, using specializations as an additional tool. We quote here a certain extension of Weil's theorem, describing the structure of "groups" defined by finite-to-finite correspondences rather than maps. We also implicitly use Cherlin's characterization of nonnilpotent groups of Morley rank 2, and Macintyre's statement that infinite fields of finite Morley rank are algebraically closed. See the introduction to this section for a description of the method.

$\S 7$. Purity of the field. In this section we compare the two systems of Zariski topologies on the field obtained in $\S 6$ (the one given by the geometry, and the algebraic one). We show first that projective space is complete in the richer topology, and that the notions of irreducibility agree. We then prove Bezout's theorem by the classical process of moving an arbitrary algebraic curve to a union of lines within an algebraic family, and conclude that as there are already enough algebraic curves to demonstrate any admissible intersection behaviour, there is no room for others.

$\S 8$. Here we show that an ample (not necessarily very ample) Zariski geometry is a finite cover of the projective line over a field, canonically. Here and in $\S 9$ we work almost entirely in the constructible category, which seems the natural context for "covers" when the geometry is not assumed complete. (Even the statement of Theorem B below is somewhat unwieldy, inasmuch as it refers to closed sets.)

$\S 9$. We analyze covers of Zariski geometries. We see that every ample Zariski geometry has a certain maximal quotient which is an algebraic curve, and the corresponding cover has strong properties. In the very ample case, the quotient can only be the full geometry, and Theorem A follows.

$\S 10$. We show that the results of $\S \S 8,9$ are nonempty by constructing a Zariski geometry as a certain double cover of an elliptic curve, which is not isomorphic to any curve and not interpretable in an algebraically closed field.

We are grateful to a number of people who read and greatly improved this paper by their cogent suggestions and corrections: Bernhard Herwig, Anand Pillay, Frank Wagner, and Martin Ziegler in Freiburg, Kobi Peterzil and Evgenia Rabinovich in Jerusalem, Sasha Ivanov and Ludomir Newelski in Wroclaw, Dave Marker in Chicago, and the referee. 


\section{Constructible sets}

If $k$ is an algebraically closed field, a constructible subset of $k^{n}$ is a finite Boolean combination of closed sets. The Tarski-Chevalley theorem states that the collection of constructible sets is closed under projections. An axiomatization of the structure of the constructible sets, analogous to the axiomatization above of the closed sets, might run as follows.

Definition. A structure is an infinite set $D$ together with a collection of subsets of $D^{n}(n=1,2, \ldots)$ closed under intersections, complements, projections and their inverses, and containing the diagonals. These are called the 0-definable sets.

Definition. A structure $D$ is strongly minimal if it satisfies: (SM) for every $n \geq 0$ and every 0-definable $C \subseteq D^{n+1}$, there exists an integer $m$ such that for all $a \in D^{n}$, letting $C(a)=\{b \in D:(a, b) \in C\}$, either $|C(a)| \leq m$ or $|D-C(a)| \leq m$.

$(\mathrm{SM})$ is analogous to the one-dimensionality axiom (Z2). It is stated in dimension one, but a powerful dimension theory can be deduced from it for arbitrary definable sets. Most simple dimension-theoretic properties of constructible sets in algebraically closed fields follow from (SM).

This definition did not arise as an attempt to describe algebraically closed fields, but rather came about naturally in a line of investigation in model theory starting from the Lowenheim-Skolem theorem. One was led to consider categorical structures, ones fully described by their cardinality and first-order theory. (Thus the complex field is determined by its cardinality, and being an algebraically closed field of characteristic 0.) Morley [Mo] showed the existence of a dimension theory for the definable sets in uncountable categorical structures; Baldwin and Lachlan [BL] discovered that such structures are controlled by strongly minimal sets within them.

It was suggested by the second author (see $[\mathrm{Z}]$ ) that examples from algebraic geometry have some importance in this context. A dividing line was introduced among the strongly minimal sets:

Definition. A strongly minimal set $D$ is locally modular if every definable family of strongly minimal subsets of $D^{2}$ has dimension at most 1 .

If $D$ arises from a Zariski geometry, this is equivalent to ampleness. The notion of a family of definable sets and its dimension will be explained in $\S 3$.

It was conjectured that nonample strongly minimal sets are essentially linear objects, whereas ample ones are essentially curves over an algebraically closed field. This would be a constructible analog to Theorem A.

The first part of this conjecture was proved in [Hr2], but a counterexample to the second was constructed in [Hr3]. However a considerable amount of technology was developed towards proving the conjecture, some of which we will use here. This technology is phrased in terms of constructible sets, rather than closed sets, since no topology was assumed to exist. Even in the Zariski context however, the constructible category is considerably more flexible than the topological one; in many situations it is quite delicate to find whether a given construction leads to a closed set, whereas the fact that it is constructible is usually trivial. We will therefore work in both categories; we explain in $\S 3$ how to translate from one to the other.

We now state the main structure theorem for ample Zariski geometries. 
Definition. Let $D_{1}, D_{2}$ be Zariski geometries. A (closed) Zariski map is a function $f: D_{1} \rightarrow D_{2}$ inducing a (closed), continuous map on $D_{1}^{n}$ for each $n$.

Theorem B. Let $D$ be an ample Zariski geometry. Then there exists an algebraically closed field $K$ and a surjective Zariski map $f: D \rightarrow \mathbf{P}^{1}(K)$. $f$ maps constructible sets to (algebraically) constructible sets, and in fact is a closed Zariski map on $D-F$ for some finite $F$.

Note that the map $f$ in Theorem B must have finite fibers: the inverse image $E_{f}$ of the diagonal on $\mathbf{P}^{1}(K)$ is a closed equivalence relation on $D$; by (Z2) each class must be finite or equal to $D$; since $\mathbf{P}^{1}(K)$ has more than one point, the former possibility must hold. One sees that $\mathbf{P}^{1}(K)$ is interpreted in $D$; it is isomorphic to $D$ modulo the closed equivalence relation $E_{f}$; and a closed subset of $\mathbf{P}^{1}(K)^{n}$ arises from a closed subset of $D^{n}$ respecting $E_{f}$. Moreover, no extra structure is induced on $\mathbf{P}^{1}(K)$ from $D$; any automorphism of $\mathbf{P}^{1}(K)$ extends to an automorphism of $D$. Thus $D$ can be viewed as a finite cover of $\mathbf{P}^{1}(K)$; however, the question of the additional structure on $D$ is delicate. Some light is thrown on the matter in $\S 9$. In $\S 10$ however, the following is shown.

Theorem C. There exists a complete, ample, one-dimensional Zariski geometry $D$ that cannot be interpreted in an algebraically closed field. In particular, if $C$ is a curve over an algebraically closed field, then every Zariski map $f: C \rightarrow D$ is constant.

Theorem B as stated does not include a canonicity statement; it is not clear for example that every automorphism of $D$ induces one of $\mathbf{P}^{1}(K)$. We will remedy this situation in the following way. Throughout the paper, we will use what might be called the full language $L(D)$ for the Zariski geometry $D$, in which an $n$-ary relation symbol corresponds to every closed subset of $D^{n}$. Note that $D$ may have nontrivial automorphisms (bijections inducing homeomorphisms in every dimension). Such automorphisms of $D$ induce automorphisms of the language $L$. We define the natural language $L$ of $D$ to be the sublanguage $L_{\text {nat }}(D)$ of $L(D)$ consisting of all automorphism invariant relations. It appears to be quite unobvious that $L_{\text {nat }}$ includes anything more than the language of pure equality. However in $\S 9$ we will show that the field of Theorem B and map $f$ of Theorem B can be chosen 0-definable in $L_{\text {nat }}$. Moreover:

Theorem B'. Let $D$ be an ample Zariski geometry, and let $L_{\text {nat }}$ be the natural language of $D$. There exist a field $K$, a smooth curve $C$ over $K$, and a surjective, finite-to-one Zariski map $f: D \rightarrow C$, all 0-definable in $L_{\text {nat }}$.

We will now show that a Zariski geometry, as defined above, is indeed a special kind of strongly minimal set; in other words that quantifier elimination holds.

Proposition 2.1. Let $D$ be a Zariski geometry. Then $D$ admits elimination of quantifiers: the projection of a constructible set is constructible.

Note that $C(a)$ is closed if $C$ is, $C(a)$ being the inverse image of $C$ under the map taking $x$ to $(a, x)$. In particular, with $C$ the diagonal, this shows every singleton is closed.

Lemma 2.2. $D^{k}$ is irreducible. 
Proof. We use induction on $k$. If $D^{k+1} \subseteq F_{1} \cup F_{2}$, let

$$
F_{i}^{*}=\left\{a \in D^{k}:(a, x) \in F_{i} \text { for all } x \in D\right\} .
$$

Clearly $F_{i}^{*}$ is closed. For any $a \in D^{k}, F_{i}(a)=\left\{x:(a, x) \in F_{i}\right\}$ is closed, and $D=$ $F_{1}(a) \cup F_{2}(a)$, so by irreducibility of $D, F_{i}(a)=D$ for some $i$. Thus $F_{1}^{*} \cup F_{2}^{*}=D^{k}$, so $F_{i}^{*}=D^{k}$ for some $i$, so $F_{i}=D^{k+1}$.

Lemma 2.3. Let $\pi: D^{n} \rightarrow D^{k}$ be a projection, $C \subseteq D^{n}$ closed, and $F=\operatorname{cl}(\pi C)$. If $C$ is irreducible, so is $F$. If $F$ is irreducible, then $F=\operatorname{cl}\left(\pi C^{\prime}\right)$ for some irreducible component $C^{\prime}$ of $C$.

Proof. Let $F=F_{1} \cup F_{2}, F_{i}$ closed. Then $\pi^{-1} F_{i}$ is closed, $C=\pi^{-1} F_{1} \cup \pi^{-1} F_{2}$, so $C=\pi^{-1} F_{1}$ (say). Thus $F=\operatorname{cl}\left(\pi \pi^{-1} F_{1}\right) \subseteq F_{1}$. Conversely suppose $F$ is irreducible and let $C=C_{1} \cup C_{2}$. Then $F=\operatorname{cl}(\pi C)=\operatorname{cl}\left(\pi C_{1}\right) \cup \operatorname{cl}\left(\pi C_{2}\right)$, so $F=\operatorname{cl}\left(\pi C_{i}\right)$ for some $i$.

Lemma 2.4. Let $E \subseteq D^{n}$ and $C \subseteq E \times D$ be closed. Suppose $C(a)$ is finite for some $a \in E$. Then $C(a)$ is finite for all $a \in E$ outside of a proper closed subset of $E$.

Proof. If $C(a)$ is infinite, then $C(a)=D$. So it suffices to prove that $\{a: C(a)$ $=D\}$ is closed. But this set is the intersection over all $d \in D$ of $\{a:(a, d) \in C\}=$ (d) $C$, which is closed.

The following lemma generalizes (Z3).

Lemma 2.5 (Dimension theorem). Let $C_{1}, C_{2}$ be closed irreducible subsets of $D^{n}$, $\operatorname{dim}\left(C_{i}\right)=d_{i}$. Then every component of $C_{1} \cap C_{2}$ has dimension at least $d_{1}+d_{2}-n$.

Proof. Let $\Delta_{i}$ be the diagonal: $x_{i}=x_{n+i}$ in $D^{2 n}$, and let $\Delta=\bigcap_{i} \Delta_{i}$. There is an isomorphism between $C_{1} \cap C_{2}$ and $\left(C_{1} \times C_{2}\right) \cap \Delta$. As in $2.2 C_{1} \times C_{2}$ is irreducible, and is easily seen to have dimension at least $d_{1}+d_{2}$. Hence it suffices to show that every component of $C \cap \Delta$ has dimension $\geq \operatorname{dim}(C)-n$, where $C$ is a closed irreducible subset of $D^{2 n}$. This follows by applying (Z3) to the intersections with the diagonals $\Delta_{i}$, in succession.

Lemma 2.6. Let $C \subseteq D^{n}$ be irreducible. Let $\pi: D^{n} \rightarrow D^{k}$ be a projection.

(a) If $\operatorname{cl}(\pi(C))=D^{k}$, then $\operatorname{dim}(C) \geq k$.

(b) If $\pi^{-1}(a) \cap C$ is finite and nonempty for some a, then $\operatorname{dim}(C) \leq k$.

(c) $\operatorname{dim}(C)=k$ iff there exists a projection satisfying (a) and (b).

Proof. (a) By induction on $k$. We have $\pi(C) \supseteq\left(D^{k}-F\right)$ for some proper closed subset $F$ of $D^{k}$. For $a \in D$, let $F(a)=\left\{y \in D^{k-1}:(a, y) \in F\right\}$. If for all $a \in D$, $F(a)=D^{k-1}$, then $F=D^{k}$; a contradiction. Choose $a \in D$ such that $F(a)$ is a proper closed subset of $D^{k-1}$. Let $C^{\prime}=\left\{x \in C: \pi(x) \in\{a\} \times D^{k-1}\right\}$. Then $C^{\prime}$ is a proper closed subset of $C$. Let $\theta: D^{k} \rightarrow D^{k-1}$ be the projection, $\pi^{\prime}=\theta \pi$. Clearly $\pi^{\prime}\left(C^{\prime}\right)$ contains $D^{k-1}-F(a)$. Since $D^{k-1}$ is irreducible, $\operatorname{cl}\left(D^{k-1}-F(a)\right)=$ $D^{k-1}$, and by $2.3 \mathrm{cl} \pi^{\prime}\left(C^{\prime \prime}\right)=D^{k-1}$ for some component $C^{\prime \prime}$ of $C^{\prime}$. By induction $\operatorname{dim}\left(C^{\prime \prime}\right) \geq k-1$, so $\operatorname{dim}(C) \geq k$.

(b) Pick $a$ such that $\pi^{-1}(a) \cap C$ is finite. Let $\pi_{i}: D^{k} \rightarrow D$ be the $i$ th projection, and let $T_{i}=\left(\pi_{i} \pi\right)^{-1}\left(\pi_{i} a\right)$. Let $C_{0}=C, C_{i+1}=\left(C_{i} \cap T_{i+1}\right)$. Then $C_{k}=\pi^{-1}(a) \cap C$, so $\operatorname{dim}\left(C_{k}\right)=0$. Using 2.5 inductively, every component of $C_{i}$ has dimension $\geq \operatorname{dim}(C)-i$, so $\operatorname{dim}\left(C_{k}\right) \geq \operatorname{dim}(C)-k$. Thus $\operatorname{dim}(C) \leq k$. 
A map $f: X \rightarrow Y$ is generically finite-to-one if the inverse image of a generic point of $f(X)$ is finite.

Claim. Let $C$ be a proper closed irreducible subset of $D^{n}$. Then there exists a generically finite-to-one map of $C$ into $D^{n-1}$.

Proof. By induction on $n$. Consider $D^{n}$ as $D^{n-1} \times D$, and let $\pi: D^{n} \rightarrow D^{n-1}$ be the projection. If $\pi \mid C$ has a finite fiber, we are done by 2.4. Otherwise, for any $a \in \pi C, C(a)$ is an infinite closed subset of $D$, so by (Z2) it equals $D$. Hence $\pi C=\left\{x \in D^{n-1}\right.$ : for all $\left.y \in D,(x, y) \in C\right\}$ (a closed subset of $D^{n-1}$ ), and $C=\pi C \times D$. So $\pi C$ is a proper closed subset of $D^{n-1}$. By induction there exists a projection $\pi^{\prime}$ of $D^{n-1}$ to $D^{n-2}$ with a finite fiber on $\pi C$. The map $(x, y) \mapsto\left(\pi^{\prime}(x), y\right)$ from $D^{n}$ to $D^{n-1}$ satisfies the requirement.

It follows using (b) that any proper closed subset of $D^{n}$ has dimension $\leq n-1$. Hence $\operatorname{dim}\left(D^{n}\right)=n$.

Now to prove (c) we use induction on $n$. If $C=D^{n}$, then $\operatorname{dim}(C)=n$, and the identity map shows that (c) is true. If $C$ is a proper subset of $D^{n}$, then by the claim there exists $\pi_{1}: D^{n} \rightarrow D^{n-1}$ with a finite fiber on $C$. By induction there exists a projection $\pi_{2}: D^{n-1} \rightarrow D^{k}$ such that $\operatorname{cl}\left(\pi_{2}\left(\operatorname{cl}\left(\pi_{1} C\right)\right)\right)=D^{k}$, and $\pi_{2}^{-1}(a) \cap \operatorname{cl}\left(\pi_{1} C\right)$ is finite for some $a$. We have $k=\operatorname{dim}\left(\operatorname{cl}\left(\pi_{1} C\right)\right)$, and by $2.3 \operatorname{cl}\left(\pi_{1} C\right)$ is irreducible. Let $F$ be a proper closed subset of $\operatorname{cl}\left(\pi_{1} C\right)$ such that $\pi_{1} C \supseteq\left(\operatorname{cl}\left(\pi_{1} C\right)-F\right)$, and $\pi_{1}^{-1}(a) \cap C$ is finite for all $a \in \operatorname{cl}\left(\pi_{1} C\right)-F$ (possible by 2.4 and (Z1)). Then $\operatorname{dim}(F)<k$, so by (a) $\operatorname{cl}\left(\pi_{2} F\right)$ is a proper subset of $D^{k}$. Choose $a \in D^{k}$ with $a \notin \operatorname{cl}\left(\pi_{2} F\right)$, and further such that $\pi_{2}^{-1}(a)$ is finite. Then $\left(\pi_{2} \pi_{1}\right)^{-1}(a)$ is finite. Further $\pi_{2} \pi_{1} C$ contains $\pi_{2} \operatorname{cl}\left(\pi_{1} C\right)-\pi_{2} F$, hence is dense in $D^{k}$. Thus $\pi_{2} \pi_{1}$ satisfies both (a) and (b).

Proof of 2.1. We must show that if $C \subseteq D^{n} \times D$ is a closed subset, $F \subseteq C$ is a closed subset, and $\pi_{1}$ denotes the projection to $D^{n}$, then $\pi_{1}(C-F)$ is a Boolean combination of closed sets. We show this by induction on $\operatorname{dim}(C)$. Note that we can immediately reduce to the case where $C$ is irreducible.

Let $C_{1}=\operatorname{cl}\left(\pi_{1} C\right)$. Then $C_{1}$ is irreducible, and for some proper closed $H \subseteq$ $C_{1}, \pi_{1} C \supseteq C_{1}-H$.

Let $C_{0}=\left\{x \in D^{n}: \forall y .(x, y) \in C\right\}$, and $F_{0}=\left\{x \in D^{n}: \forall y .(x, y) \in F\right\}$. Then $C_{0}, F_{0}$ are closed and $C_{0} \subseteq C_{1}$. The case $C_{0}=C_{1}$ is trivial, since then $\pi_{1}(C-F)=C_{0}-F_{0}$.

Let $F_{1}=\mathrm{cl}\left(\pi_{1} F\right)$. If $F_{1}$ is a proper subset of $C_{1}$, then so is $F_{2}=F_{1} \cup H$, and $\left(F_{2} \times D\right) \cap C$ is a proper subset of $C$, and hence has smaller dimension. Thus by induction $\pi_{1}\left(\left(F_{2} \times D\right) \cap C-F\right)$ is a Boolean combination of closed sets. Hence so is $\pi_{1}(C-F)=\pi_{1}\left(\left(F_{2} \times D\right) \cap C-F\right) \cup\left(C_{1}-F_{2}\right)$.

The remaining case is $C_{1}=F_{1}, C_{0} \neq F_{1}$. In this case we claim that $C=F$. Since $C$ is irreducible, it suffices to show that $\operatorname{dim}(C)=\operatorname{dim}(F)$. In fact $\operatorname{dim}(C)=$ $\operatorname{dim}\left(C_{1}\right)$ and $\operatorname{dim}(F)=\operatorname{dim}\left(F_{1}\right)$. This follows from the characterization of $\operatorname{dim}(F)$ in Lemma 2.6. (If $\pi$ is a generically surjective map on $F_{1}$ with a finite fiber, then $\pi_{1} \pi$ is the same for $F$.)

Corollary 2.7. $D$ is strongly minimal.

Proof. Let $E \subseteq D^{n} \times D$ be a definable set. We must show that $E(a)$ is finite or co-finite, with a uniform bound for all $a \in D^{n}$. We may take $E=C-F$, with $C, F$ closed. If $C(a)$ is finite, then $E(a)$ is finite, with the same bound. The result is immediate from (Z2) applied to $C$ and to $F$. 


\section{MODEL THEORETIC PRELIMINARIES}

We list here some basic facts concerning first-order structures. However in certain sections we will require more familiarity with these notions than can probably be attained here. We refer the reader to $[\mathrm{FJ}]$ or $[\mathrm{CK}]$ for the general notions, and to [Pi1] for results connected with Morley's dimension for definable sets.

Structures and maps. A structure is a set $M$ together with a collection of subsets of $M^{n}$ for each $n$ (the "0-definable sets") closed under intersections, complements, projections and their inverses, and containing the diagonals. We assume that the collection of 0-definable sets is indexed in some way, as $\left\{R_{i}: i \in I\right\}$; the indexing is called the language; we also write $R_{i}(M)$ for $R_{i}$ as a subset of $M^{n}$. One can think of $R_{i}(M)$ as the set of $M$-points of the definable set $R_{i}$, as one does for varieties. A map $f: M \rightarrow N$ is one preserving the $R_{i}$, so that $\left(x_{1}, \ldots, x_{n}\right) \in R_{i}(M)$ iff $\left(f x_{1}, \ldots, f x_{n}\right) \in R_{i}(N)$. We always write $f\left(x_{1}, \ldots, x_{n}\right)$ for $\left(f x_{1}, \ldots, f x_{n}\right)$.

A definable subset of $M^{n}$ is one of the form $C(a)=\{y:(a, y) \in C\} \subseteq M^{n}$, where $C$ is a 0 -definable subset of $M^{k+n}$ and $a \in M^{k}$. We say that a set is $A$-definable if it has the form $C(a)$ for some $a$ from $A$. An elementary substructure of $M$ is a subset $N$ of $M$ with the following property: if $C$ is a nonempty $N$-definable set, then $C \cap N \neq \emptyset$. We then consider $N$ as a structure whose 0 -definable sets are the restrictions to $N$ of the 0 -definable sets in $M$.

If $A \subseteq M$ and $c \in M^{k}$, the type of $c$ over $A, \operatorname{tp}(c / A)$, is the collection of all $A$-definable sets containing $c$.

Because of the quantifier elimination proved for Zariski structures, a definable set is just a constructible set in this context (a Boolean combination of closed sets).

Universal domain. A basic theorem of model theory states that every structure $M$ is an elementary substructure of a larger structure $M^{*}$ with the following properties:

3.1. (compactness) Let $C$ be a countable collection of definable subsets of $M^{*}$, with the finite intersection property. Then the intersection of all members of $C$ is nonempty.

3.2. (homogeneity) Let $f: A_{1} \rightarrow A_{2}$ be a bijective map between countable subsets of $M^{*}$. Then $f$ extends to an automorphism of $M^{*}$.

In [FJ] such a structure is called an enlargement of $M$. "Countable" in 3.1, 3.2 can be replaced with various other notions of "smallness", such as "of cardinality smaller than a given cardinal $\kappa$ ". This involves considerations that are irrelevant here, though strictly speaking they may be needed if one starts with an uncountable Zariski geometry. See [CK] for this, and also for certain uniqueness statements (on saturated models).

It is useful to state a version of 3.2 using types:

$3.2^{\prime}$. Let $A$ be countable. If $b, c$ are elements of $M^{*}$ with the same type over $A$, then there exists an automorphism of $M^{*}$ fixing $A$ and taking $b$ to $c$.

Thus a type over $A$ corresponds to an orbit of $\operatorname{Aut}\left(M^{*} / A\right)$. Sometimes we will confound the distinction. We say that $a, b$ are conjugate over $A$ if they have the same type over $A$.

Let $A_{0}$ be a countable set. It follows from 3.1 that the following properties of a set $A$ are equivalent: (i) $A$ is invariant under $\operatorname{Aut}\left(M^{*} / A_{0}\right)$. (ii) $A$ is a union of complete types over $A_{0}$. We call $A A_{0}$-normal in this case. 
When $M$ is stable in the sense of Shelah, in particular when the structure has finite Morley rank in the sense to be explained below, the homogeneity in 3.2 can be extended. We will use this on one isolated occasion.

Definition. Let $B$ be a countable subset of $M^{*}$. $A \subseteq M^{*}$ is $B$-normal if $A$ is invariant under $\operatorname{Aut}\left(M^{*} / B\right)$.

3.3. If $A_{1}, A_{2}$ are $B$-normal, $B$ is countable, and $f:\left(B \cup A_{1}\right) \rightarrow\left(B \cup A_{2}\right)$ is a map fixing $B$, then $f$ extends to an automorphism of $M^{*}$.

Convention A. All elements $a, b, \ldots$ mentioned without qualification are assumed to belong to $M^{*}$ (not necessarily $M$ ). All sets of elements $A, B, \ldots$ are assumed to be countable, or normal over some countable set.

Imaginary elements. One can also consider many-sorted structures, which consist of a number of sets $M_{i}$ (called "sorts") together with a collection of subsets of their various Cartesian products, with the same closure properties. We will use a canonical many-sorted structure associated with a given structure $M$. It consists of $M$ together with additional "imaginary sorts", all implicitly contained in $M$.

Definition. A structure $N$ is interpretable in a structure $M$ if there exists a 0 definable subset $U$ of $M^{n}$ and a surjective map $j: U \rightarrow N$ such that for any 0-definable $D \subseteq N^{k}, j^{-1}(D)$ is a 0 -definable subset of $U^{k} \subseteq\left(M^{n}\right)^{k}$.

If $N$ is interpretable in $M$, then any statement concerning definable subsets of $N$ can be converted to one about $M$. In particular $j^{-1}(=)$ is a 0 -definable equivalence $E$ relation on $U$, and $N$ can be identified with a structure whose universe is $U / E$. This leads to the formation of Shelah's many-sorted structure $M^{\mathrm{eq}}$, containing a copy of each structure interpretable in $M$ (with the maximal possible set of 0 definable relations).

Suppose $D$ is a 0 -definable subset of $M^{n}$ for some $n$, and $E$ is a 0 -definable subset of $M^{n+n}$, which is an equivalence relation on $D$. Then we call $s=(D, E)$ a sort, and we let $M_{s}=D / E$. We have a map $\pi_{s}: D \rightarrow M_{s}$. We consider $M_{s}$ as a structure whose 0-definable sets are those subsets $X$ of $M_{s}^{k}$ whose pullback by $\pi_{s}$ is 0 -definable in $M$. This construction is functorial; if $M^{*}$ is an elementary extension of $M$, then $M_{s}^{*}$ is an elementary extension of $M_{s}$ in the natural way.

We will need to work in order to get some theory of imaginaries in the Zariski context. In the constructible category however no difficulty arises. In particular the notion of dimension defined below applies also to imaginary sorts.

Convention B. When elements or sets are introduced without qualification, they are always assumed to lie in $M_{s}^{*}$ for some imaginary sort $S$ (and not necessarily in $M)$.

Algebraic and definable closure. A definable function is a function whose graph is a definable set. A subset of $M^{*}$ (or $M^{*}$ eq) is definably closed if it is closed under the definable functions (in any number of variables). We write $\operatorname{dcl}(A)$ for the definable closure of $A$. If $M$ is an algebraically closed field, $A \subseteq M$, then $\operatorname{dcl}(A)$ is the smallest perfect subfield of $M$ containing $A$.

3.4. $a \in \operatorname{dcl}(A)$ iff $a$ is fixed by $\operatorname{Aut}\left(M^{*} / A\right)$.

Proof. Suppose $a$ is fixed by $\operatorname{Aut}\left(M^{*} / A\right)$. Then by $3 \cdot 2^{\prime}$ there is no $a^{\prime} \neq a$ with the same type over $A$. Hence the type of $a$ over $A$, together with the definable 
set $\{x: x \neq a\}$, has empty intersection. By 3.1 some finite subset has empty intersection. It follows that $\{a\}$ is a finite intersection of $A$-definable sets, so it is $A$-definable. Say $\{a\}=C\left(a_{1}, \ldots, a_{k}\right)$, with $C$ a 0 -definable set and $a_{1}, \ldots, a_{k}$ from $A$. Let $E=\left\{\left(x_{1}, \ldots, x_{k}\right)\right.$ : there exists a unique $y$ with $\left.\left(x_{1}, \ldots, x_{k}, y\right) \in C\right\}$. Let $C^{\prime}=\left\{\left(x_{1}, \ldots, x_{k}, y\right):\left(x_{1}, \ldots, x_{k}, y\right) \in C\right.$ and $\left.\left(x_{1}, \ldots, x_{k}\right) \in E\right\}$. Then $C^{\prime}$ is definable and is the graph of a definable function $f$, and $f\left(a_{1}, \ldots, a_{k}\right)=a$.

3.4 gives a very useful Galois theory between definably closed sets, and certain subgroups of $\operatorname{Aut}\left(M^{*}\right)$.

We say that $a \in \operatorname{acl}(A)$ if $a \in C$ for some finite, $A$-definable set $C$. $A$ is algebraically closed if $A=\operatorname{acl}(A)$. Similarly to 3.4 one can show:

3.5. $a \in \operatorname{dcl}(A)$ iff the orbit of $a$ under $\operatorname{Aut}\left(M^{*} / A\right)$ is finite.

Dimension and rank. The notion of dimension can be defined in the constructible category; it is customarily called Morley rank. One defines recursively the class of definable sets of Morley rank at least $n$. For $n=0$, it is the class of all nonempty definable sets. A definable set $D$ has rank at least $n+1$ iff for all $m, D$ contains $m$ pairwise disjoint subsets of rank at least $n$.

We say that $D$ has Morley rank $n$ if it has rank at least $n$, but not at least $n+1$. We write $\operatorname{rk}(D)=n$. We also write $\operatorname{rk}(a / B)=n$ to mean that $a$ lies in some $B$-definable set of rank $n$, but not of any smaller rank. We say then that the type of $a$ over $B$ has rank $n$.

Conventionally, $\operatorname{rk}(\emptyset)=-\infty$.

If we begin with a Zariski structure $X$, it can easily be shown that the Morley rank of a definable set $E$ of $X^{k}$ is the dimension of the closure of $E$ in $X^{k}$ (for example this follows from 2.6).

$\operatorname{rk}(a / B)$ is the dimension of the locus of $a$ over $B$. In an algebraically closed field, it equals the transcendence degree of the field generated by $B$ and $a$, over that generated by $B$.

3.6. Suppose $M$ has Morley rank 1 , and let $M_{s}$ be an imaginary sort (possibly $\left.M^{n}\right)$. Then $M_{s}$ has finite Morley rank. Moreover, for all $A, a, b$ :

(a) (additivity) $\operatorname{rk}(a b / A)=\operatorname{rk}(b / A)+\operatorname{rk}(a / A \cup\{b\})$.

(b) $\operatorname{rk}(a / A \cup\{b\}) \leq \operatorname{rk}(a / A)$.

(c) $\operatorname{rk}(a / A)=0$ iff $a \in \operatorname{acl}(A)$.

Independence. We say that $a_{1}, \ldots, a_{n}$ are independent over $A$ if

$$
\operatorname{rk}\left(a_{1} \cdots a_{n} / A\right)=\sum_{i} \operatorname{rk}\left(a_{i} / A\right) .
$$

Note that the notions of independence over $A$, and over $\operatorname{acl}(A)$, are the same.

We also say that $a, b$ are free over $A$ when there are just two elements. If $M^{*}$ is an algebraically closed field, $A$ a subfield, this is the same as saying that the fields $A(a), A(b)$ are free over $A$.

The following comes from 3.6:

3.7. (a) If $a$ is free from $b$ over $A$, and from $c$ over $A \cup\{b\}$, then $a$ is free from $(b, c)$ over $A$.

(b) If $a_{1}, \ldots, a_{n}$ are independent over $A$, then so is any permutation.

Shelah's "finite equivalence relation theorem" can be stated as follows, using Convention B. 
3.8. Suppose $A=\operatorname{acl}(A) \subseteq B, \operatorname{tp}(a / A)=\operatorname{tp}(b / A)$, and each of $a, b$ are free from $B$ over $A$. Then $\operatorname{tp}(a / B)=\operatorname{tp}(b / B)$.

If $p$ is a type over a set $A$ and $A \subseteq B$, a generic (over $B$ ) realization of $p$ is any element $a$ whose type over $A$ is $p$, and such that $a$ is free from $B$ over $A$. Thus 3.8 states that if $A$ is algebraically closed, then any two generic realizations of $p$ are conjugate. We will also say that $a_{1}, \ldots, a_{n}$ are mutually generic over $A$ if each is generic, and they are independent over $A$.

Families. In the constructible category the deep problems associated with moduli varieties do not exist. Let $Y$ be a definable set. A family of definable subsets of $Y$ is a definable subset $C$ of $P \times Y$, where $P$ is a definable set, such that $C(a)$ has constant Morley rank $k(a \in P)$. Write $Z \equiv_{k} Z^{\prime}$ iff the symmetric difference of $Z, Z^{\prime}$ has Morley rank smaller than $k$, and define an equivalence relation on $P: a \equiv b$ iff $C(a) \equiv{ }_{k} C(b)$. This equivalence relation is definable; thus $P^{*}=P / \equiv$ can be viewed as a definable set (of imaginary elements). The dimension of the family is the Morley rank $P^{*}$.

3.9. There exists $C^{*} \subseteq P^{*} \times Y$ such that for $a \in P, C(a) \equiv_{k} C^{*}(a / \equiv)$.

Thus any given family can be replaced with a normal one, for which $\equiv$ is the identity. (Cf. [HH], normalization theorem.)

\section{$Z$-structures.}

3.10. Let $M$ be a structure of finite Morley rank, together with a distinguished generation family of definable sets, referred to as closed. We call $M$ a $Z$-structure if (Z0) holds.

By assumption, every definable set is a Boolean combination of closed sets; it follows that $\operatorname{cl}(\operatorname{cl}(X)-X)$ has dimension $<\operatorname{dim}(\operatorname{cl}(X))$. (Z1) follows automatically from the quantifier elimination condition on a structure (closure under projections).

From this point of view, a Zariski geometry is a strongly minimal $Z$-structure satisfying the dimension theorem (Z3). The condition (Z3) is the only one without a natural model-theoretic meaning. Indeed the interpretation of the field was achieved in this framework in 1989 for complete Zariski geometries, but was neglected in part because condition (Z3) appeared too restrictive.

\section{Specializations}

In this section we introduce the language of specializations, and prove their basic properties in Zariski geometries. This language is entirely equivalent to the description in terms of closed sets, but for many purposes more convenient to work with.

We regard the Zariski geometry $D$ as a first-order structure; the relations are the closed subsets of $D^{n}$. We will not carefully distinguish between the closed subset $F \subseteq D^{n}$ and the predicate naming it in the language. We observe that the Zariski structure is carried over into elementary extensions (see $[\mathrm{CK}]$ ).

Definition. Let $D^{*}$ be an elementary extension of $D$. Let $F$ be a closed subset of $D^{n}$. Then $F\left(D^{*}\right)$ is a subset of $D^{* n}$. Sets of this form are called 0-closed sets. If $A$ is a subset of $D^{*}$, an $A$-closed set in $D^{n}$ is one of the form $F(a)$, where $F \subseteq D^{m} \times D^{n}$ is a 0 -closed set, and $a=\left(a_{1}, \ldots, a_{m}\right) \in A^{m}$.

Definition. The locus of $c$ over $A$ is the smallest $A$-closed set containing $a$. The rank of $c$ over $A$ is the dimension of the locus of $c$ over $A$. 
Proposition 4.1. $D^{*}$ together with the collection of closed subsets of $D^{* n}$ forms a Zariski geometry. It is complete if $D$ is complete.

Before proving the proposition we make some remarks. Since $D$ is strongly minimal, so is $D^{*}$, and we may use Morley rank. The only difficulty is to verify that every closed set is a finite union of closed irreducible sets, and that a proper closed subset of a closed irreducible set has smaller Morley rank.

For any $a$, the topology determined by the $a$-closed sets is easily seen to be Noetherian. (If $a \in D^{* n}$ and $F_{i}(a)$ is a family of $a$-closed sets in $D^{* m}$, then $F_{i}$ can be viewed as a subset of $D^{n+m}$, and some finite intersection $K$ of the $F_{i}$ is contained in every $F_{j}$; then $K(a)$ is a finite intersection of the $F_{i}(a)$ and is contained in every $F_{j}(a)$.) We call it the $a$-topology. The union of all the $a$-topologies will be called the absolute topology (though we do not yet know it is a topology).

Lemma 4.2. Let $D_{1}$ be a closed, irreducible subset of $D^{k}$, and let $E$ be a closed irreducible subset of $D_{1} \times D^{m}$. Then there exists a proper closed subset $F_{1}$ of $D_{1}$ such that for every $a \in D_{1}-F_{1}$, every component of $E(a)$ has dimension $\geq \operatorname{dim}(E)-\operatorname{dim}\left(D_{1}\right)$.

Proof. The case $D_{1}=D^{k}$ is immediate from 2.5, with $F_{1}=\emptyset$. In general let $r=\operatorname{dim}\left(D_{1}\right)$. By 2.1 and strong minimality, there exists a projection $\pi: D^{k} \rightarrow D^{r}$ and a proper closed subset $F_{1}$ of $D_{1}$ such that $\pi$ is finite-to-one on $D_{1}-F_{1}$. So we may think of $D^{k}$ as $D^{r} \times D^{l}$. Let $a \in D_{1}-F_{1}$ and write $a=\left(a_{r}, a_{l}\right)$. Then $E(a)=E\left(a_{r}\right)\left(a_{l}\right)$ in the obvious sense. By the special case mentioned above, every component $U$ of $E\left(a_{r}\right)$ has dimension $\geq \operatorname{dim}(E)-r$. But by the property of $\pi$, the projection of $U$ to $D^{l}$ is finite; since $U$ is irreducible, it has one element, namely $a_{l}$. Thus $U=\left\{a_{l}\right\} \times U\left(a_{l}\right)$. Evidently $E(a)$ is covered by the sets $U\left(a_{l}\right)$ as $U$ ranges through the components of $E\left(a_{r}\right)$; so every component of $E(a)$ has dimension $\geq \operatorname{dim}(E)-r$.

Remark $4.2^{\prime}$. Suppose

$D_{1}$ can be covered by open sets $G$, such that each $G$

admits a map to some $D^{k}$ with finite fibers.

Then the conclusion (and proof) of 4.2 are valid for any $a \in D_{1}$. It follows that if $(*)$ holds for all closed sets, then so does the property

For all irreducible $E,\left\{a \in D_{1}: \operatorname{dim} E\left(a^{\prime}\right) \geq t\right\}$ is a relatively closed subset of $\left\{a \in D_{1}: \operatorname{dim} E\left(a^{\prime}\right) \geq 0\right\}$.

By Noether's integral basis theorem, every variety has the property $(*)$. It appears that $(*)$ follows for all ample Zariski geometries, using Theorem B, and hence that (\#) holds. But we have not been able to find a direct proof.

Lemma 4.3. Let $C \subseteq D^{* n}$ be a closed set. Assume $C$ is invariant under $\operatorname{Aut}\left(D^{*} / A\right)$, where $A$ is a finite set. Then $C$ is an $A$-closed set.

Proof. Let $a=\left(a_{1}, \ldots, a_{m}\right)$ enumerate $A$. Fix for a moment a point $c$ of $C$. Let $C^{*}$ be the smallest 0 -closed set containing $(a, c)$, and $D_{1}$ the locus of $a$. Let $k=\operatorname{dim}\left(D_{1}\right), l=\operatorname{dim}\left(C^{*}\right)-k$. Let $F_{1}$ be a subset of $D_{1}$ as in 4.2 . By 4.2 , for every $D$-closed set $C^{\prime}$ and every $a^{\prime} \in D_{1}-F_{1}$, either $C^{*}\left(a^{\prime}\right) \subseteq C^{\prime}$ or $\operatorname{rk}\left(C^{*}\left(a^{\prime}\right)-C^{\prime}\right) \geq l$. Transferring this statement to $D^{*}$, for every closed set $C^{\prime}$ and every $a^{\prime} \in D_{1}-F_{1}$, either $C^{*}\left(a^{\prime}\right) \subseteq C^{\prime}$ or $\operatorname{rk}\left(C^{*}\left(a^{\prime}\right)-C^{\prime}\right) \geq l$. Let $a \in D_{1}, \operatorname{rk}(a / D)=k$. We claim 
that $C^{*}(a) \subseteq C$. Suppose for contradiction that this is false, and let $b \in C^{*}(a)-C$, $\operatorname{rk}(b / D, a)=l$. Then $\operatorname{rk}(a b)=k+l=\operatorname{dim}\left(C^{*}\right)$. Thus $(a, b)$ is a generic point of $C^{*}$, hence is conjugate to $(a, c)$ under $\operatorname{Aut}\left(D^{*}\right)$. However, $c \in C$; a contradiction. This shows that $C^{*}(a) \subseteq C$. Moreover $c \in C^{*}(a)$. Thus every point of $C$ lies in some $A$-closed set contained in $C$. By compactness, there is a finite number of $A$-closed sets contained in $C$ whose union is $C$. Thus $C$ is $A$-closed.

Ziegler's proof of 4.1. The referee has communicated to us a proof of 4.1 suggested by Ziegler, much shorter than ours. We leave our proof in since the intermediate claims seem to throw some light on the situation. We indicate however Ziegler's proof, with the flavor of order type $\omega^{2}$ rather than $\omega$. One considers directly a supposed strictly descending chain $C_{i}\left(a_{i}^{*}\right)$ of closed subsets of $D^{* n}$. Say $a_{i}^{*} \in D^{* m(i)}$. Then since $D$ is an elementary submodel, for every $M$ there exist $a_{i} \in D^{m(i)}$ such that $C_{i}\left(a_{i}\right)$ is strictly descending, $i \leq M$. However for a fixed sequence $C_{i} \subseteq D^{n+m(i)}$ one shows this is not the case. Indeed at each $i$ either the number of irreducible components of maximal dimension goes down, or the dimension goes down. The only problem is to bound the number of irreducible components of the new smaller dimension, in the latter case. This can be done because at each point one is dealing with sets in a fixed number of families $C_{i}(a)$.

Proof of 4.1. Claim 1. Let $X$ be a definable set in $D^{* n}$. Then there exists a closed set $Y$ containing $X$, with $\operatorname{rk}(Y-X)<\operatorname{rk}(X)$. If $X$ is $b$-closed, so is $Y$.

Proof. We may write $X=U(b)$, where $U$ is a 0 -definable set, and $\operatorname{rk}(U)=$ $\operatorname{rk}(X)+\operatorname{rk}(b)$. Let $V$ be a 0 -closed set containing $U$, such that $\operatorname{rk}(V-U)<\operatorname{rk}(U)$. Note that $\operatorname{rk}(V-U) \geq \operatorname{rk}(b)+\operatorname{rk}((V-U)(b))$. Let $Y=V(b)$; then $Y$ contains $X$, and

$\operatorname{rk}(Y-X)=\operatorname{rk}((V-U)(b)) \leq \operatorname{rk}(V-U)-\operatorname{rk}(b)<\operatorname{rk}(U)-\operatorname{rk}(b)=\operatorname{rk}(X)$.

Call a closed set $X$ weakly irreducible if there is no decomposition $X=Y \cup Z$, $Y, Z$ closed of rank equal to $\operatorname{rk}(X), \operatorname{rk}(Y \cap Z)<\operatorname{rk}(X)$.

Claim 2. Every closed set $X$ is a finite union of closed, weakly irreducible sets, of the same rank as $X$.

Proof. Let $m$ be the Morley multiplicity of $X$. Then one cannot express $X$ as a union $X=X_{1} \cup \cdots \cup X_{m+1}$, with $X_{i}$ closed of $\operatorname{rank}$ equal to $\operatorname{rk}(X)$, and $\operatorname{rk}\left(X_{i} \cap X_{j}\right)<\operatorname{rk}(X)$. Let $X=X_{1} \cup \cdots \cup X_{l}$ be a maximal such expression, $l \leq m$. Then it is clear that each $X_{i}$ is weakly irreducible.

Claim 3. Let $U$ be a closed subset of $D^{m+k}, Y$ a definable subset of $D^{m}$, and let $V=\left\{x \in D^{k}\right.$ : for all $\left.y \in Y,(y, x) \in U\right\}$. Then $V$ is an intersection of finitely many sets $U\left(y_{i}\right), y_{1}, \ldots, y_{m} \in Y$. Moreover, if $U$ and $Y$ vary through a definable family of definable sets, then the bound $m$ can be chosen independent of $Y$.

Proof. The first assertion is immediate from the Noetherianity of $D$. For the second, note that $V=U\left(y_{1}\right) \cap \cdots \cap U\left(y_{m}\right)$ iff $U\left(y_{1}\right) \cap \cdots \cap U\left(y_{m}\right) \cap\left(D^{k}-V\right)$ is empty. Moreover, as $Y$ and $U$ vary through a definable family, so does $V$. Thus we are reduced precisely to the following statement:

Let $F$ be a definable family of definable subsets of $D^{k}$. There

exists an integer $m$ such that if the intersection of finitely many

sets from $F$ is empty, then already the intersection of $m$ of

them is empty. 
This is just the negation of Shelah's finite cover property. It holds in strongly minimal structures by [BL] and [Sh1]. Alternatively, one can prove it as follows in this context. Let $W$ range through intersections of finitely many of the sets in $F$. By reverse induction on $r \leq k$, show that if $\operatorname{rk}(W)=r$, there are only finitely many possibilities for $\operatorname{Mult}(W)$; and that if $W=\bigcap_{i} X_{i}$, then already the intersection of some subfamily of bounded size has the same rank and multiplicity. The key point is that one can uniformly bound multiplicities, i.e., for any $Y^{\prime} \subseteq Z \times D^{l}$, there exists an integer $k$ such that $Y^{\prime}(b)$ always has multiplicity at most $k$.

Claim 4. If $C$ is $a$-closed, $a$-irreducible, and weakly irreducible, then $C$ is absolutely irreducible.

Proof. Suppose otherwise. Then for some $e, C=C^{\prime}(e) \cup F(e)$, where $C^{\prime}, F$ are 0 -closed, and precisely one of the sets $C^{\prime}(e), F(e)$ - say the former - has rank equal to $\operatorname{rk}(C)$. Let

$$
Y=\left\{e^{\prime}: C=C^{\prime}\left(e^{\prime}\right) \cup F\left(e^{\prime}\right), \operatorname{rk}\left(C^{\prime}\left(e^{\prime}\right)\right)=\operatorname{rk}(C), \operatorname{rk}\left(F\left(e^{\prime}\right)\right)<\operatorname{rk}(C)\right\} .
$$

Then $Y$ is an $a$-definable set.

Let $C \#=\left\{x\right.$ : for all $\left.e^{\prime} \in Y, x \in C^{\prime}\left(e^{\prime}\right)\right\}$. So $C \#$ is an intersection of closed subsets of $C$ of the form $C-F\left(e^{\prime}\right), \operatorname{rk}\left(F\left(e^{\prime}\right)\right)<\operatorname{rk}(C)$. Now Claim 3 persists to $D^{*}$, so $C \#$ is a finite intersection of sets of this form. Thus $C \#$ is closed, and $\operatorname{rk}(C \#)=\operatorname{rk}(C)$. But clearly $C \#$ is $a$-definable. By $4.3, C \#$ is $a$-closed. By $a$ irreducibility of $C, C=C \#$. Thus $C^{\prime}(e)=C$, and $F(e)=\emptyset$. This shows that $C$ is irreducible.

It now follows easily, by induction on rank, that every closed set is a finite union of closed irreducible sets.

Claim 5. Let $U$ be irreducible and $e$-closed. Then $U=U^{*}(e)$ for some irreducible 0 -closed set $U^{*}$. Moreover $\operatorname{rk}\left(U^{*}\right)=\operatorname{rk}(U)+\operatorname{rk}(e)$.

Proof. Let $U^{*}$ be the smallest 0 -closed set such that $U=U^{*}(e)$. It is easy to see that $U$ is irreducible, and that $e$ is a generic point of the appropriate projection of $U^{*}$, so that the rank statement also holds.

Now if $U \subseteq V$ are irreducible, $\operatorname{rk}(U)=\operatorname{rk}(V)$, say $U, V$ are $e$-closed; then by Claim 5, $U=U^{*}(e), V=V^{*}(e)$ with $U^{*}, V^{*}$ irreducible, and $\operatorname{rk}\left(U^{*}\right)=\operatorname{rk}\left(V^{*}\right)$; moreover one sees easily that $U^{*} \subseteq V^{*}$. Thus $U^{*}=V^{*}$, so $U=V$.

This finishes the proof of 4.1 .

Convention. We thus work with $D^{*}$ instead of $D$. In other words, among the closed sets, we are given also a class of 0 -closed sets, such that every closed set has the form $C(a)$ for some $a$ and some 0 -closed set $C$, and such that $D$ is saturated when considered as a structure, with the class of 0 -closed sets as the language.

Definition. Let $M, N$ be models of $\operatorname{Th}(D), A \subseteq N$. A map $f: A \rightarrow M$ is a specialization if $c \in A^{k}$ and $P(c)$ for every 0 -closed set $P$ of $k$-tuples, then $P(f c)$.

If $A=\left(a_{i}: i \in I\right), B=\left(b_{i}: i \in I\right)$, and the indexing is understood, we write $A \rightarrow B$ if the map $a_{i} \mapsto b_{i}$ is a specialization.

A specialization is nothing more than a homomorphism, with respect to the language whose atomic relations are the 0 -closed sets.

The following proposition will not be used at all, but we include it as a complement. It was important in the original treatment of the subject. Recall that a Zariski geometry is complete if all projection maps are closed. In algebraic geometry, one first proves the lemma on extensions of places, and deduces the completeness of projective space; here we go in the opposite direction. 
Proposition 4.4 (Extensions of specializations lemma). Let $D$ be a complete Zariski geometry. Let $M, N$ be two models of $\operatorname{Th}(D)$. Let $A \subseteq B \subseteq N$. Then any specialization $f: A \rightarrow M$ extends to a specialization of $N$ to $M$.

Proof. Given $a \in N$, we must extend $f$ to $A \cup\{a\}$. Consider the smallest $A$-closed set of which $a$ is a member. This set has the form $\{x:(x, b) \in Q\}$ where $Q$ is a 0 -closed set and $b$ is from $A$. By completeness, the projection $P$ of $Q$ to the "second" coordinate is closed. Since $f$ is a specialization, $f b \in P$, and since $M$ is a model of the theory of $D$, one can find $a^{\prime}$ in $N$ with $\left(a^{\prime}, f b\right) \in Q$. Now one verifies easily that this gives a specialization.

Definition. A closed set is single-dimensional (of pure dimension $r$ ) if all its components have the same dimension $(r)$.

Notation 4.5. (i) $\operatorname{rk}\left(a \rightarrow a^{\prime}\right)=\operatorname{rk}(a)-\operatorname{rk}\left(a^{\prime}\right)$.

(ii) $a \rightarrow_{b} a^{\prime}$ if $a b \rightarrow a^{\prime} b$.

(iii) $\left(a_{i}: i \in I\right) \rightarrow\left(a_{i}^{\prime}: i \in I\right)$ for infinite $I$ if for every finite $I_{0} \subseteq I,\left(a_{i}: i \in\right.$ $\left.I_{0}\right) \rightarrow\left(a_{i}^{\prime}: i \in I_{0}\right)$.

Notation 4.6. Let $C \subseteq D_{1} \times D_{2}, D_{1}$ irreducible. Let $r=\operatorname{rk} C(a)$ for generic $a \in D_{1}$. Let $E_{0}\left(C, D_{1}\right)=\left\{x \in D_{1}: \operatorname{rk}(C(x))>r\right\}, E\left(C, D_{1}\right)=\operatorname{cl}\left(E_{0}\left(C, D_{1}\right)\right)$. Say that $b \rightarrow b^{\prime}$ is exceptional with respect to $a$ if $b^{\prime} \in E\left(C, D_{1}\right)$, where $D_{1}$ is the locus of $b$ and $C$ is the locus of $(b, a)$.

In the following proposition, we assume for the moment that $D_{1}, D_{2}$ are relatively closed subsets of powers of $D$, i.e., open subsets of their closures and $c$ is a tuple of elements of $D$. Later we will define manifolds, and then $D_{1}$ may be chosen to be any manifold, and $D_{2}$ a closed subset of any manifold (with the same proof). We will use the following assumption:

(4.7A): $C$ is a closed irreducible subset of $D_{1} \times D_{2} ; D_{1}, D_{2}$ are also irreducible; $D_{2}$ may be embedded as a closed subset of some $Y$, such that the dimension theorem holds in $D_{1} \times Y^{m}$ for all $m$.

Proposition 4.7. Assume the situation of (4.7A). Let $a \in D_{1}$ be generic and $r=\operatorname{rk}(C(a)) \geq 0$. Let $(a, c) \rightarrow\left(a^{\prime}, c^{\prime}\right)$, with $a^{\prime} \notin E\left(C, D_{1}\right)$. Let $b^{\prime} \in C\left(a^{\prime}\right)$. Then there exists $b \in C(a), \operatorname{rk}(b / a c)=r$, with $(a, b, c) \rightarrow\left(a^{\prime}, b^{\prime}, c^{\prime}\right)$.

Remark 4.8. Observe the following special case of 4.7. If (4.7A) holds, if $C(a)$ is finite of size $m$ for generic $a \in D_{1}$, if $a^{\prime} \in D_{1}$, and if $a^{\prime} \notin E\left(C, D_{1}\right)$, then $\left|C\left(a^{\prime}\right)\right| \leq|C(a)|$. In fact there exists a specialization extending $a \rightarrow a^{\prime}$ and carrying a subset of $C(a)$ onto $C\left(a^{\prime}\right)$.

Lemma 4.9. Suppose $D_{1}$ is irreducible, $C_{i} \subseteq D_{1} \times D_{2}(i=1,2)$ closed, with $C_{1}$ single-dimensional. Let $r=\operatorname{dim}\left(C_{1}(a)\right)$ for generic $a \in D_{1}$, and let $E=$ $E\left(C_{1}, D_{1}\right)$. Suppose $r \geq 0$. Suppose $C_{1}(a) \subseteq C_{2}(a)$ for generic $a \in D_{1}$. Then $C_{1} \subseteq C_{2} \cup\left(E \times D_{2}\right)$.

Proof. By single-dimensionality of $C_{1}$ it suffices to show that every element of $C_{1}$ of maximal rank is in $C_{2} \cup\left(E \times D_{2}\right)$. Let $(a, b)$ be such an element of $C_{1}$. If $a \in E$, then $(a, b) \in E \times D_{2}$. If $a \notin E$, then $\operatorname{rk}(b / a) \leq r$, so $\operatorname{rk}(a, b) \leq \operatorname{rk}(a)+r$. But $r \geq 0$ so for generic $a^{\prime} \in D_{1}$ and $b^{\prime} \in C_{1}\left(a^{\prime}\right), \operatorname{rk}\left(a^{\prime}, b^{\prime}\right)=\operatorname{rk}\left(D_{1}\right)+r$. By maximality of $\operatorname{rk}(a, b), \operatorname{rk}(a)=\operatorname{rk}\left(D_{1}\right)$, so $a$ is a generic element of $D_{1}$. Thus by assumption $(a, b) \in C_{2}$. 
Lemma 4.10. (a) Let $C \subseteq D_{1} \times D_{2}, D_{1}$ irreducible of dimension $r_{1}, C$ of pure dimension $r+r_{1}$. Assume the dimension theorem holds in $D_{1} \times D_{2}$. Then for $a \in D_{1}-E\left(C, D_{1}\right), C(a)$ is empty or of pure dimension $r$.

(b) Suppose $F \subseteq D_{1} \times D_{2}, G \subseteq D_{1} \times D_{3}, D_{2} \subseteq E_{2}, D_{3} \subseteq E_{3}$, and the dimension theorem holds in $D_{1}^{2} \times E_{2} \times E_{3}$. Let

$$
H=\{(a, b, c):(a, b) \in F,(a, c) \in G\} .
$$

Then every component of $H$ has dimension at least $\operatorname{dim}(F)+\operatorname{dim}(G)-\operatorname{dim}\left(D_{1}\right)$.

Proof. (a) We may assume $C$ is irreducible. Note that for generic $a \in D_{1}, r_{1}+$ $\operatorname{rk}(C(a))=\operatorname{rk}(a)+\operatorname{rk}(C(a)) \leq \operatorname{rk}(C)=r+r_{1}$ so $\operatorname{rk}(C(a)) \leq r$. Hence for $a \in$ $D_{1}-E\left(C, D_{1}\right)$ we also have $\operatorname{rk}(C(a)) \leq r$. But by the dimension theorem, every nonempty component of $C \cap\{a\} \times D_{2}$ has dimension $\geq\left(r+r_{1}\right)-\operatorname{rk}\left(D_{1}\right)=r$. Equivalently every component of $C(a)$ has dimension $\geq r$. Thus $C(a)$ is of pure dimension $r$.

(b) Let $H^{\prime}=\{(a, a, b, c):(a, b) \in F,(a, c) \in G\}$. Then $H^{\prime}$ is isomorphic to $H$. On the other hand $H^{\prime}$ can be viewed, within $D_{1}^{2} \times E_{2} \times E_{3}$, as the intersection of the diagonal $\Delta=\left\{(a, a, b, c): a \in D_{1}, b \in E_{2}, c \in E_{3}\right\}$ with a closed set isomorphic to $F \times G$. Since $\Delta$ has codimension equal to $\operatorname{dim}\left(D_{1}\right)$, the dimension theorem shows that every component of $H^{\prime}$ has dimension at least $\operatorname{dim}(F)+\operatorname{dim}(G)-\operatorname{dim}\left(D_{1}\right)$.

Lemma 4.11. Assume (4.7A), and that $\operatorname{cl}\left(\pi_{1} C\right)=D_{1}$. Let $a \in D_{1}$ be generic, $r=$ $\operatorname{rk}(C(a))$. Let $a_{i}=\left(a, a_{i}^{*}\right)$ be a tuple containing a, and $C_{i} \subseteq\{(x, y, z):(x, z) \in C\}$ a closed irreducible with $C(a)=\bigcup_{i} C_{i}\left(a_{i}\right)$. Let $\left(a^{\prime}, a_{1}^{\prime}, \ldots, a_{m}^{\prime}\right)$ be a specialization of $\left(a, a_{1}, \ldots, a_{m}\right), a^{\prime} \notin E\left(C, D_{1}\right)$. Then $C\left(a^{\prime}\right)=\bigcup_{i} C_{i}\left(a_{i}^{\prime}\right)$.

Proof. Let $F_{0}$ be the locus of $\left(a, a_{1}, \ldots, a_{m}\right)$,

$F_{1, i}$ the locus of $\left(a, a_{1}, \ldots, a_{m}, b_{i}\right)$, where $b_{i}$ is a generic point of $C_{i}\left(a_{i}\right)$,

$F_{1}=\bigcup_{i} F_{1, i}$, and

$F_{2}^{\prime}=\left\{\left(a, a_{1}, \ldots, a_{m}, b\right):\left(a, a_{1}, \ldots, a_{m}\right) \in F_{0}\right.$ and $\left.(a, b) \in C\right\}$.

Let $F_{2}$ be the union of all components of $F_{2}^{\prime}$ not contained in

$$
\left\{\left(x, x_{1}, \ldots, x_{m}, y\right): x \in E\left(C, D_{1}\right)\right\} .
$$

Claim. $F_{2}$ is single-dimensional.

Proof. Let $\left(x, x_{1}, \ldots, x_{m}, y\right)$ be a generic element of some component of $F_{2}$. Then $x \notin E\left(C, D_{1}\right)$. So $\operatorname{rk}(y / x) \leq r$. Thus $\operatorname{rk}\left(x, x_{1}, \ldots, x_{m}, y\right) \leq \operatorname{rk}\left(F_{0}\right)+r$. On the other hand we may apply the dimension theorem in $D_{1}^{2} \times Y^{f}$ (where $Y$ is from (4.7A), and the locus of $\left(x_{1}, \ldots, x_{m}, y\right)$ is a closed subset of $\left.Y^{f}\right)$. Specifically, we intersect $F_{0} \times C$ within an appropriate $D_{1}$-diagonal, to obtain a copy of $F_{2}^{\prime}$. The $D_{1^{-}}$ diagonal is defined by the equality of two projections to $D_{1}$, and has codimension $\operatorname{dim}\left(D_{1}\right)$. Thus every nonempty component of $F_{2}^{\prime}$ has dimension greater than or equal to $\operatorname{rk}\left(F_{0}\right)+\operatorname{rk}(C)-\operatorname{rk}\left(D_{1}\right) \geq \operatorname{rk}\left(F_{0}\right)+r$.

By assumption,

$$
F_{1}\left(a, a_{1}, \ldots, a_{m}\right)=F_{2}\left(a, a_{1}, \ldots, a_{m}\right),
$$

and $\left(a^{\prime}, a_{1}^{\prime}, \ldots, a_{m}^{\prime}\right) \notin E\left(F_{2}, F_{0}\right)$. By 4.9 (and the fact that $\left.F_{1} \subseteq F_{2}\right)$ we have

$$
F_{1}\left(a^{\prime}, a_{1}^{\prime}, \ldots, a_{m}^{\prime}\right)=F_{2}\left(a^{\prime}, a_{1}^{\prime}, \ldots, a_{m}^{\prime}\right) \text {. }
$$

In other words $C\left(a^{\prime}\right)=\bigcup_{i} C_{i}\left(a_{i}^{\prime}\right)$. 
Proof of Proposition 4.7. Let $C_{1}$ be the locus of $(a, c), C^{*}=\{(x, z, y):(x, z) \in$ $\left.C_{1},(x, y) \in C\right\}$. Let $C_{i}(i=1, \ldots, d)$ be the irreducible components of $C^{*}$ satisfying rk $C_{i}(a, c)=r$. By 4.3 (or the proof of 4.1, Claims 1 and 4) every component of $C(a)$ has dimension $r$; hence $C(a)=\bigcup_{i} C_{i}(a, c)$. By 4.11, $C\left(a^{\prime}\right)=\bigcup_{i} C_{i}\left(a^{\prime}, c^{\prime}\right)$; so $b^{\prime} \in C_{i}\left(a^{\prime}, c^{\prime}\right)$ for some $i$. Let $b$ be a generic point of $C_{i}(a, c)$. Since $C_{i}$ is irreducible and $(a, c, b)$ is a generic point of $C_{i},(a, b, c) \rightarrow\left(a^{\prime}, b^{\prime}, c^{\prime}\right)$.

Lemma 4.12. If $\operatorname{rk}\left(b \rightarrow b^{\prime}\right) \leq 1$, then $b \rightarrow b^{\prime}$ is nonexceptional with respect to any $a$.

Proof. Let $C$ be the locus of $b a, A$ the locus of $b, E=E(C, A)$. Then $\operatorname{dim}(E) \leq$ $\operatorname{dim}(A)-2$. For let $b^{*}$ be a generic point of $E$. Then $b^{*} \in E_{0}(C, A), \operatorname{sod} \operatorname{dim}\left(C\left(b^{*}\right)\right) \geq$ $\operatorname{dim}(C(b))+1$. Hence if $a^{*}$ has maximal rank in $C\left(b^{*}\right)$, then $\operatorname{rk}\left(a^{*} b^{*}\right) \geq \operatorname{dim}(E)+$ $\operatorname{dim} C(b)+1$. But $a^{*} b^{*}$ is not generic in $C$, so $\operatorname{rk}\left(a^{*} b^{*}\right)<\operatorname{dim}(C)=\operatorname{dim}(A)+$ $\operatorname{dim} C(b)$. So $\operatorname{dim}(E)<\operatorname{dim}(A)-1$. Since $\operatorname{rk}\left(b^{\prime}\right) \geq \operatorname{rk}(b)-1, b^{\prime} \notin E$.

Lemma 4.13. Let $a \rightarrow a^{\prime \prime}$; suppose $a(1) \neq a(2), a^{\prime \prime}(1)=a^{\prime \prime}(2)$. Then there exists $a^{\prime}, a \rightarrow a^{\prime} \rightarrow a^{\prime \prime}, a^{\prime}(1)=a^{\prime}(2)$, with $\operatorname{rk}\left(a \rightarrow a^{\prime}\right)=1$.

Proof. Immediate from the dimension theorem. $a^{\prime}$ is any generic point of the component of the intersection of $\operatorname{locus}(a)$ with the diagonal $x(1)=x(2)$ containing the locus of $a^{\prime \prime}$.

\section{Elimination of IMAginaries}

In the present section we will define the notion of a "manifold" over a Zariski geometry $D$. The goal is to obtain a large collection of universes in which the dimension theorem remains true. (It is not true in an arbitrary closed set of $D^{n}$.) We will first identify "special" imaginary sorts, where the dimension theorem holds (5.1); then identify "regular subsets" of these, on which it perseveres (5.4); and finally show such sets can be glued together (the key is 5.5, 5.10).

We will show that any structure interpretable in $D$ is piecewise a manifold ("elimination of imaginaries"). If it carries a definable group operation, one also has a global result: Weil's group chunk theorem, stating that a constructible group has a unique manifold structure in this sense, is valid here. This is the basis for the transition between the categories.

Definition. We define a special imaginary sort. Let $H$ be a subgroup of $\operatorname{Sym}(n)$. Let $H$ act naturally on $D^{n}$. Let $S_{H}=D^{n} / H$. Then $S_{H}$ is the special imaginary sort corresponding to $H$.

Remark. If $D$ is a rational curve over an algebraically closed field, the notion of a $D$-manifold can be defined without introducing the new sorts; but if we start with another curve, we will not otherwise get a sufficiently general notion.

The following is an unpublished result of Lascar and Pillay, in the strongly minimal case. We state it in that case, but note that the proof given goes through when $D$ is definable by a possible infinite set of formulas in some saturated structure, and every definable subset meets $D$ in a finite or cofinite set.

Lemma 5.1. Let $D$ be a strongly minimal set, and suppose acl $(\emptyset)$ is infinite in $D$. For any imaginary sort $S$ there exists a partition of $S$ into a finite number of 0-definable sets $X_{i}$, special sorts $S_{i}$, and 0-definable injective maps $f_{i}: X_{i} \rightarrow S_{i}$. 
Equivalently, for any imaginary element e there exists an element $e^{\prime}$ of a special sort, such that $\operatorname{dcl}(e)=\operatorname{dcl}\left(e^{\prime}\right)$.

Proof. We have $e=\left(a_{1}, \ldots, a_{n}\right) / E$ for some $a_{1}, \ldots, a_{n} \in D$ and some 0-definable equivalence relation $E$. We may renumber so that $a_{1}, \ldots, a_{j}$ are in $\operatorname{acl}(e)$, but the others are not. Choose the elements $a_{i}$ so that $j$ is as large as possible. If $j<n$, then $a_{j+1}$ is not algebraic over $e$. Hence for all but finitely many elements $x_{j+1}$ of $D$ there exist $x_{j+2}, \ldots, x_{n}$ such that $e=\left(a_{1}, \ldots, a_{j}, x_{j+1}, \ldots, x_{n}\right) / E$. In particular there exists $a_{j+1}^{\prime}$ in $\operatorname{acl}(\emptyset) \cap D$ such that for some $a_{j+1}^{\prime}, \ldots, a_{n}^{\prime}, e=$ $\left(a_{1}, \ldots, a_{j}, a_{j+1}^{\prime}, \ldots, a_{n}^{\prime}\right) / E$. But this contradicts the maximality of $j$. Hence $j=n$, so each $a_{i}$ is algebraic over $e$.

Now let $a=\left(a_{1}, \ldots, a_{n}\right) \in D^{n}$, and let $C$ be the set of all conjugates of $a$ over $e$. Clearly $C$ has no proper conjugates over $e$, and (since $e=a^{\prime} / E$ for any $\left.a^{\prime} \in C\right), e$ has no proper conjugates by automorphisms leaving $C$ invariant. Thus $\operatorname{dcl}(e)=\operatorname{dcl}(\{C\})$.

$C$ has some finite number $m$ of elements. It can thus be viewed as a member of the collection of $m$-element subsets of $D^{n}$. This collection can be identified with the set of $m$-tuples of $D^{n}$, modulo the action of $H=\operatorname{Sym}(m)$ on $\left(D^{n}\right)^{m}$. Now regarding $H$ as a subgroup of $\operatorname{Sym}(n \cdot m)$, we see that $e$ is equivalent to an element of the special sort $S_{H}$.

Observe that if $S, T$ are special sorts, then $S \times T$ may naturally be identified with another special sort. We give each special sort a topology as follows. If $S=S_{H}=D^{n} / H$, let $\pi_{H}: D^{n} \rightarrow H$ be the natural map. We call $X \subseteq S$ closed if $\pi_{H}^{-1}$ is closed in $D^{n}$.

The same definition could have been made for any sort $D^{n} / E$, where $E$ is a closed equivalence relation. However, the dimension theorem would not in general hold in $D^{n} / E$. For example, suppose $E$ is the equivalence relation identifying two points on $D$; so the map $D \rightarrow D / E$ is generically $1-1$, but has one fiber of size 2 . This contradicts Remark 4.8; tracing through the proof we see that the dimension theorem fails in $(D / E) \times D^{2}$.

We show however that in the special sorts, the dimension theorem continues to hold.

Lemma 5.2. Suppose $D$ is a Zariski geometry, and let $S$ be a special sort, of dimension $n$. Let $C_{1}, C_{2}$ be closed irreducible subsets of $S, \operatorname{dim}\left(C_{i}\right)=d_{i}$. Then every component of $C_{1} \cap C_{2}$ has dimension at least $d_{1}+d_{2}-n$.

Proof. Let $S=S_{H}=D^{n} / H$, and let $\pi=\pi_{H}$ be the projection. Observe that $\pi$ is a closed map: if $X$ is closed in $D^{n}$, then $\pi^{-1} \pi X=\bigcup_{\sigma \in H} \sigma X$ is closed, hence $\pi X$ is closed. Let $C_{1}, C_{2}$ be closed irreducible subsets of $S$, $\operatorname{dim}\left(C_{i}\right)=d_{i}$, and let $X$ be a component of $C_{1} \cap C_{2}$. Let $D_{i}$ be a component of $\pi^{-1} C_{i}$ projecting onto $C_{i}$. (Any component of maximal dimension will do.) If $x \in C_{1} \cap C_{2}$, then $x=\pi y_{i}$ with $y_{i} \in D_{i}$. Since $\pi y_{1}=\pi y_{2}$ we have $\sigma y_{2}=y_{1}$ for some $\sigma \in H$, so $y_{1} \in\left(D_{1} \cap \sigma D_{2}\right)$. Thus $C_{1} \cap C_{2} \subseteq \bigcup_{\sigma} \pi\left(D_{1} \cap \sigma D_{2}\right)$. Let $\left\{Y_{i}\right\}$ be the connected components of the sets $D_{1} \cap \sigma D_{2}, \sigma \in H$. By the dimension theorem in $D^{n}$, each $Y_{i}$ has dimension $\geq d_{1}+d_{2}-n$. It follows that $\pi Y_{i}$ is connected, of dimension at least $d_{1}+d_{2}-n$. Since $C_{1} \cap C_{2}$ is contained in $\bigcup_{i} \pi Y_{i}$, the connected components of $C_{1} \cap C_{2}$ are among the sets $\pi Y_{i}$; hence all have the appropriate dimension.

Write $\operatorname{codim}_{X} Y$ for $\operatorname{dim}(X)-\operatorname{dim}(Y)$. Let $\Delta_{C}$ denote the diagonal on $C \times C$. 
Definition. Let $C \subseteq S=D^{n}$ be a closed irreducible set of dimension $k$. A point $p \in C$ is regular on $C$ if there exists a closed irreducible set $G \subseteq S \times S$, $\operatorname{codim}_{S \times S}(G)=\operatorname{dim}(C)$, such that $\Delta_{C}$ is the unique component of $G \cap(C \times C)$ passing through $(p, p)$.

If $C$ is any closed set in $D^{n}, p$ is regular if $p$ lies in a unique component of $C$, whose dimension equals $\operatorname{dim}(C)$, and is regular on that component.

Let $S$ be a special sort, $\pi: D^{n} \rightarrow S$ the defining projection, and let $C$ be a closed subset of $S$. A point $p \in C$ is regular on $C$ if $p=\pi p^{*}$ for some regular $p^{*}$ on $h^{-1} C$.

Remark. This appears closely related to the notion of local complete intersection (l.c.i.) in algebraic geometry; see [Ha], 8.22, 8.23. If $S$ is a smooth variety, and if $C$ is a closed subvariety and is l.c.i. at an open neighborhood of $p$, then $\Delta_{C}$ is l.c.i. at $(p, p)$, and hence $p$ is regular on $C$ ([Ha], 8.22.2). The converse also seems plausible. For our present purposes regularity is a technical notion capable of yielding a sufficient supply of sets on which the dimension theorem is valid.

Lemma 5.3. Let $S$ be a special sort, $C$ a closed subset. Then the set of regular points of $C$ forms a dense open subset of $C$.

Proof. Since the projection $\pi: D^{n} \rightarrow S$ is a closed and open map, even on $C$, we are reduced to the case $S=D^{n}$. The set of points of $C$ lying on only one component of $C$, of dimension equal to $\operatorname{dim}(C)$, is dense open, so we may assume $C$ is irreducible.

Claim. There exists a closed irreducible set $G \subseteq S \times S, \operatorname{codim}_{S \times S}(G)=$ $\operatorname{codim}_{S}(C)$, such that $\Delta_{C}$ is a component of $G \cap(C \times C)$.

Proof. Let $\pi: D^{n} \rightarrow D^{k}$ be a projection, $k=\operatorname{dim}(C)$, such that $\pi$ is finite-to-one on a dense open subset $C^{*}$ of $C$. Let $G$ be the inverse image of the diagonal of $D^{k}$ under the map $(\pi, \pi)$. Then $G$ is closed irreducible, of codimension $k$ in $D^{n} \times D^{n}$. Clearly $\Delta_{C} \subseteq G \cap(C \times C)$. Moreover

$$
\operatorname{dim}\left(G \cap\left(C^{*} \times C^{*}\right)\right)=\operatorname{dim}\left\{(x, y) \in C^{*}: \pi x=\pi y\right\}=\operatorname{dim} C^{*}
$$

since $\pi$ is finite-to-one on $C^{*}$. Thus $\Delta_{C}$ has the same dimension as $G \cap\left(C^{*} \times C^{*}\right)$. On the other hand,

$$
\operatorname{dim}\left(\Delta_{C} \cap\left((C \times C)-\left(C^{*} \cap C^{*}\right)\right)\right)=\operatorname{dim}\left(C-C^{*}\right)<\operatorname{dim}\left(\Delta_{C}\right) .
$$

So $\Delta_{C}$ is a component of $G \cap(C \times C)$.

Let $G$ be as in the claim, and let $G^{*}$ be the union of all components of $G \cap(C \times C)$ other than $\Delta_{C}$. Then $\operatorname{dim}\left(\Delta_{C} \cap G^{*}\right)<\operatorname{dim}(C)$, so $\Delta_{C}-G^{*}$ is a dense open subset of $\Delta_{C}$, and $\pi_{1}\left(\Delta_{C}-G^{*}\right)=\left\{p:(p, p) \in\left(\Delta_{C}-G^{*}\right)\right\}$ is a dense open subset of $C$. The set of regular points is the union over all such $G$ of $\pi_{1}\left(\Delta_{C}-G^{*}\right)$.

Lemma 5.4. Let $S_{i}$ be special sorts, $C_{i}$ a closed irreducible subset of $S_{i}$, and $p_{i}$ a regular point of $C_{i}$. Let $U, V$ be closed subsets of $C=\prod_{i} C_{i}$, and let $E$ be a component of $U \cap V$, passing through $p=\left(p_{1}, \ldots, p_{n}\right)$. Then

$$
\operatorname{dim}(E) \geq \operatorname{dim}(U)+\operatorname{dim}(V)-\operatorname{dim}(C) .
$$

Proof. Viewing $S=\prod_{i} S_{i}$ as a special sort $D^{n} / H$, it is easy to see that $p$ is regular on $C$. Let $\pi: D^{n} \rightarrow S$ be the defining map of $S$ as a special sort, $p=\pi p^{*}$, with $p^{*}$ regular on $\pi^{-1} C$. $p^{*}$ lies on a unique component $C^{*}$ of $\pi^{-1} C$, with $\operatorname{dim}\left(C^{*}\right)=$ $\operatorname{dim}(C)$, and is regular on $C^{*}$. Let $E^{*}$ be a component of $U^{*} \cap V^{*}$, where $U^{*}, V^{*}$ 
are components of $\pi^{-1} U, \pi^{-1} V$. Now $U \cap V$ is covered by the sets $\pi E^{*}$, and hence the components of $U \cap V$ containing $p$ are included among the sets $\pi E^{*}$, where $E^{*}$ passes through some $p^{*}$ with $p=\pi p^{*}$. The action of $H$ shows that all $p^{*}$ are regular on $\pi^{-1} C$, and hence all $E^{*}$ passing through a point $p^{*}$ satisfy the required inequality, given the special case $S=D^{n}$. We are thus reduced to this case.

By the definition of a regular point, there exists a closed irreducible subset $G$ of $S \times S$ of codimension equal to $\operatorname{dim}(C)$, such that $\Delta_{C}$ is the unique component of $G \cap C^{2}$ passing through $(p, p)$. Let $E \#$ be a component of $G \cap(U \times V)$ containing $\Delta_{E}$. By the dimension theorem in $S^{2}$,

$$
\operatorname{dim}(E \#) \geq \operatorname{dim}(U \times V)-\operatorname{codim}_{S \times S}(G)=\operatorname{dim}(U)+\operatorname{dim}(V)-\operatorname{dim}(C) .
$$

Now $E \#$ is contained in $G \cap C^{2}$ and passes through $(p, p)$, hence is contained in $\Delta_{C}$. Thus $E \# \subseteq \Delta_{U \cap V} \subseteq G \cap(U \times V)$. It follows that $E \#$ is a component of $\Delta_{U \cap V}$, and thus equal to $\Delta_{E}$. Hence

$$
\operatorname{dim}(E)=\operatorname{dim}\left(\Delta_{E}\right)=\operatorname{dim}(E \#) \geq \operatorname{dim}(U)+\operatorname{dim}(V)-\operatorname{dim}(C) .
$$

Definition. A subset $U$ of a special sort is called regular if $U$ is open in its closure $C$, and every point of $U$ is regular on $C$.

Lemma 5.5. Let $U, V$ be irreducible regular subsets of special sorts, and let $\Gamma$ be a closed irreducible subset of $U \times V$. Assume $\Gamma$ is the graph of a function $g: U \rightarrow V$. Then $g$ is continuous: for any closed $G \subseteq V, g^{-1} G$ is closed in $U$.

Proof. Let $F$ be an irreducible component of $\operatorname{cl}\left(g^{-1} G\right)$. We will show that $F \subseteq$ $g^{-1} G$. It follows that $g^{-1} G=\operatorname{cl}\left(g^{-1} G\right)$ is closed.

$g^{-1} G$ is the projection to $U$ of $(U \times G) \cap \Gamma$, so it is a constructible set. Hence so is $F \cap g^{-1} G$. Now $g^{-1} G$ is contained in the union of all the components of $\operatorname{cl}\left(g^{-1} G\right)$ other than $F$, together with $\operatorname{cl}\left(F \cap g^{-1} G\right)$; hence $\operatorname{cl}\left(F \cap g^{-1} G\right)=F$, so there exists a closed set $F^{\prime} \subseteq F, \operatorname{dim}\left(F^{\prime}\right)<\operatorname{dim}(F)$, such that $F-F^{\prime} \subseteq g^{-1} G$. Since $\Gamma$ is the graph of a function, $\operatorname{dim}\left(\left(F^{\prime} \times V\right) \cap \Gamma\right) \leq \operatorname{dim}\left(F^{\prime}\right)<\operatorname{dim}(F)$. If $(a, b) \in(F \times V) \cap \Gamma$ and $a \notin F^{\prime}$, then $b=g(a) \in G$, so $(a, b) \in(U \times G)$. Thus every component of $(F \times V) \cap \Gamma$ not contained in $(F \times V) \cap \Gamma$ is contained in $(U \times G)$. But by the dimension theorem, all the components of $(F \times V) \cap \Gamma$ have dimension at least $\operatorname{dim}(F)+\operatorname{dim}(V)+\operatorname{dim}(\Gamma)-\operatorname{dim}(U \times V)=\operatorname{dim}(F)$. Hence all the components are contained in $U \times G$, so $(F \times V) \cap \Gamma \subseteq(U \times G)$. Thus if $a \in F$, then $(a, g(a)) \in(U \times G)$, so $a \in g^{-1} G$. This shows that $F \subseteq g^{-1} G$.

Remark 5.6. The irreducibility assumption on $\Gamma$ is necessary, even if $g$ is a bijection. For example, let $a=(1: 0: 0), b=(0: 1: 0)$ in two-dimensional projective space $P^{2}$.

Let $L_{1}, L_{3}$ be the lines defined by: $x=0, z=0$, respectively. Let $U=P^{2}-\{a, b\}$. Consider the bijection $f$ between $P^{2}-L_{1}-L_{3}$ and itself, of degree 2 , given by: $(x: y: z) \rightarrow\left(z^{2}: x y: x z\right)$. Let $\Phi$ be the graph of $f$. The closure of $\Phi$ in $\left(P^{2}\right)^{2}$ is

$$
\Phi_{*}=L_{1} \times a \cup L_{3} \times b \cup a \times L_{1} \cup b \times L_{3} \cup \Phi .
$$

Thus $\Phi^{*} \cap U^{2}=\Phi$. We may extend $f$ to a bijection $g$ on $U$, by the identity on $U \cap\left(L_{1} \cup L_{3}\right)$. 
Manifolds and the many-sorted geometry. It is convenient to consider manysorted Zariski geometries. These consist of a collection of "sorts" $D_{i}$, with "projection maps" $D_{i} \rightarrow D_{j}$ for various $i, j$. One assumes that if $D_{i}, D_{j}$ are sorts, then so is $D_{i} \times D_{j}$, and the two natural projections are among the given maps. The structure consists of a topology on each sort, such that the obvious parallels of (Z0), (Z1), and 2.5 hold. We assume the existence of a "fundamental sort" $D$, such that for every closed set $F$ (in any sort) there exist $n$ and a closed set $C \subseteq D^{n} \times F$ separating points on a dense open subset of $F$. (Z2) is assumed to hold for closed subsets of $D_{i} \times D$, for any sort $D_{i}$.

We now define a $D$-manifold. This is intended to be a topological space $X$, modelled locally on regular subsets of special sorts of $D$. We admit only connected manifolds.

Definition. Let $D$ be a Zariski geometry. By a $D$-manifold we will mean a set $X$ and a finite number of injective maps $g_{i}: U_{i} \rightarrow X$, such that:

(i) $\bigcup_{i} g_{i} U_{i}=X$

(ii) each $U_{i}$ is an irreducible regular subset of some special sort,

(iii) for each $i, j,\left\{(x, y) \in U_{i} \times U_{j}: g_{i} x=g_{j} y\right\}$ is a closed irreducible subset of $U_{i} \times U_{j}$, projecting onto a nonempty open subset of $U_{i}$ and of $U_{j}$.

If $X$ is a manifold, we obtain a topology on $X$, namely the collection of all sets $W \subseteq X$ such that $g_{i}^{-1} W$ is open in $U_{i}$ for each $i$. By (iii) and 5.5, each $g_{i}$ is then a homeomorphism between $U_{i}$ and $g_{i} U_{i}$. One sees easily that with this topology $X$ is an irreducible Noetherian space, of the same dimension as any of the sets $U_{i}$. Moreover the dimension theorem holds in $X$.

If $X, X^{\prime}$ are manifolds, there is a natural manifold structure on $X \times X^{\prime}$ (covered by the sets $U_{i} \times U_{j}$ ). This gives a topology on $X \times X^{\prime}$. In particular we get a topology on $X^{n}$ for each $n$, and more generally on all products of manifolds. It is easy to verify that the collection of all manifolds, topologized in this way, forms a many-sorted Zariski geometry.

Definition. Let $X_{1}, X_{2}$ be manifolds. A map $\alpha: X_{1} \rightarrow X_{2}$ is a morphism if the graph of $\alpha$ is closed irreducible in $X_{1} \times X_{2}$.

Remark. This should not be confused with the natural notion of a homomorphism of induced Zariski structures; we refer to the latter as a specialization.

Lemma 5.7. Let $X_{1}, X_{2}$ be manifolds, and let $\left\{U_{i}\right\},\left\{V_{j}\right\}$ be open covers of $X_{1}, X_{2}$ respectively. Let a map $\alpha: X_{1} \rightarrow X_{2}$ be given. Then the following are equivalent:

(i) $\alpha$ is a morphism.

(ii) For each $j, \alpha^{-1} V_{j}$ is open in $X_{1}$, and the restriction of $\alpha$ to $\alpha^{-1} V_{j}$ is a morphism.

(iii) For each $i, \alpha \mid U_{i}$ is a morphism.

Proof. (i) $\rightarrow$ (iii) and (i) $\rightarrow$ (ii) are clear. We show that (iii) $\rightarrow$ (i); applying this to the cover $\left\{\alpha^{-1} V_{j}\right\}$, (ii) $\rightarrow$ (i) also follows. To show that (iii) $\rightarrow($ i), note that the graph $\Gamma$ of $\alpha$ is the union over $i$ of the graph $\Gamma_{i}$ of $\alpha \mid U_{i}$. Hence it is closed. If $\Gamma$ is the union of two closed sets $X, X^{\prime}$, then $\Gamma_{i} \subseteq X$ or $\Gamma_{i} \subseteq X^{\prime}$, by irreducibility of $\Gamma_{i}$. But $\Gamma_{i}$ is dense in $\Gamma$, so $\Gamma=X$ or $\Gamma=X^{\prime}$.

Lemma 5.8. Let $U, V$ be two manifolds, $f: U \rightarrow V$ a morphism. Then $f$ is continuous. 
Proof. By 5.5 and 5.7.

Lemma 5.9. If $f_{i}: U_{i} \rightarrow V_{i}$ are morphisms $(i=1,2)$, then so is $f: U_{1} \times U_{2} \rightarrow$ $V_{1} \times V_{2}$ defined by $f\left(u_{1}, u_{2}\right)=\left(v_{1}, v_{2}\right)$.

Proof. Clear.

Lemma 5.10. The composition of two morphisms is a morphism.

Proof. Let $f: U \rightarrow V, g: V \rightarrow W$ be morphisms. Then the maps $(f, 1): U \times W \rightarrow$ $V \times W$ given by $(f, 1)(u, w)=(f u, w)$ and $(g, 1): V \times W \rightarrow W \times W$ given by $(g, 1)(v, w)=(g v, w)$ are both morphisms. Hence they are continuous, so their composition $(g f, 1)$ is continuous. But the inverse image of the diagonal on $W^{2}$ under $(g f, 1)$ is just the graph $\Gamma$ of $g f$. Thus this graph is closed. Let $F, G$ be the graphs of $f, g$. Then $\Gamma$ is the projection to $U \times W$ of $(F \times W) \cap(U \times G)$. Now this intersection has a unique component of dimension $\geq \operatorname{dim}(U)$, since it defines a function from $U$ to $W$ and $U$ is irreducible. But by the dimension theorem, every component has dimension $\geq \operatorname{dim}(U)$. Hence $(F \times W) \cap(U \times G)$ is irreducible, and consequently so is the continuous image $\Gamma$.

The following proof is adopted from [Hr2]. It is convenient here to drop the requirement that manifolds be connected (i.e., that the set in (iii) of the definition of manifold be nonempty). However the remark immediately following the proof will restore the situation.

Lemma 5.11. Let $G$ be a definable group in a Zariski geometry D. Then $G$ may be endowed with a manifold structure, in such a way that multiplication $G^{2} \rightarrow G$ and inversion $G \rightarrow G$ are morphisms.

Proof. This is similar to [W]. We first find a definable map $g_{0}: V_{0} \rightarrow G$, where $V_{0}$ is a regular subset of some special sort, $g_{0}$ is injective, and $g_{0} V_{0}$ has the same Morley rank and degree as $G$. We may take $g_{0}$ to be the identity. By reducing $V_{0}$ further, we may assume

There exist morphisms $m: M_{0} \rightarrow V_{0}$ and $n: V_{1} \rightarrow V_{0}$, where

$M_{0}, V_{1}$ are dense open subsets of $V_{0}^{2}$ and $V_{0}$, respectively, and $m, n$ coincide with the group multiplication and inversion on their domains.

Let $V_{2}$ be a dense open subset of $V_{0}$ such that for $x \in V_{1}$, for any $y$ generic to $x,(y, x) \in M_{0}$ and $\left(y^{-1}, y x\right) \in M_{0}$. Let $V=\left\{x: x \in V_{2} \& x^{-1} \in V_{2}\right\}, M=M_{0} \cap V^{3}$. We have:

(i) $M$ is dense open in $V \times V, m: M \rightarrow V$ is a morphism, and $m(x, y)=x y$ for $(x, y) \in M$.

(ii) For $s \in V$, for generic $x,(x, s) \in M$ and $\left(x^{-1}, x s\right) \in M$.

(iii) $V=V^{-1}$, and inversion is a morphism on $V$.

Since $V$ is a regular subset of some special sort, we may model the manifold $G$ on $V$. Let $r=\operatorname{dim}(G)$; let $a_{0}, \ldots, a_{r}$ be mutually generic elements of $G$. Then any element of $G$ lies in $a_{i} V$ for some $i$. Let $h_{i}(v)=a_{i} v$. Then the maps $h_{i}$ cover $G$. The verification that $G$ attains a manifold structure in this way, and that multiplication and inversion are morphisms, all reduce to the following claim. 
Claim. Let $a, b \in G$. Then $H_{1}=\{(x, y): a x b y \in V\}$ is open in $V \times V$, and the map $(x, y) \mapsto a x b y$ is a morphism $H_{1} \rightarrow V$.

Proof. Write $b=b_{1} b_{2}$, where $b_{1}, b_{2} \in V$. (In a stable group any element can be written as the product of two realizations of the generic type.) Suppose $a x_{0} b_{1} b_{2} y_{0} \in$ $V$. Let $c$ be an element generic to everything. So $\left(c a, x_{0}\right) \in M,\left(\operatorname{cax}_{0}, b_{1}\right) \in M$, $\left(\operatorname{cax}_{0} b_{1}, b_{2}\right) \in M$, and $\left(\operatorname{cax}_{0} b_{1} b_{2}, y_{0}\right) \in M$. (Each time because the first coordinate is generic over the second.) Moreover by (ii) applied to $a x_{0} b_{1} b_{2} y_{0}$ (an element of $V),\left(c^{-1}, \operatorname{cax}_{0} b_{1} b_{2} y_{0}\right) \in M$. Let

$$
\begin{aligned}
H_{2}=\{(x, y) \in V: & (c a, x) \in M,\left(m(c a, x), b_{1}\right) \in M, \\
& \left(m\left(m(c a, x), b_{1}\right), b_{2}\right) \in M, \\
& \left(m\left(m\left(m(c a, x), b_{1}\right), b_{2}\right), y\right) \in M, \\
& \text { and } \left.\left(c^{-1}, m\left(m\left(m\left(m(c a, x), b_{1}\right), b_{2}\right), y\right)\right) \in M\right\} .
\end{aligned}
$$

Then we have shown that $\left(x_{0}, y_{0}\right) \in H_{2} ; H_{2}$ is open, and $H_{2} \subseteq H_{1}$; and axby= $m\left(\left(c^{-1}, m\left(m\left(m\left(m(c a, x), b_{1}\right), b_{2}\right), y\right)\right)\right)$ for $(x, y) \in H_{2}$. As openness and morphismhood are local, this proves the claim.

Remark 5.12. (a) If $G$ is a connected group (i.e., has no definable subgroups of finite index), then the manifold structure on $G$ must be irreducible. For $G$ acts by translation on the set $X$ of its irreducible components. Since $X$ is finite, the subgroup $G_{0}=\{g \in G: g C=C$ for all $C \in X\}$ has finite index in $G$; it is also clear that $G_{0}$ is definable. Hence $G_{0}=G$. Thus $g C=C$ for any $g \in G, C \in X$ : so $C=G C=G$ whenever $C \neq \emptyset$.

(b) The manifold structure on $G$ is unique. For suppose $G_{1}, G_{2}$ are two manifold structures on the group $G$. We assume for convenience that $G$ is connected. The identity is a definable group isomorphism $h: G_{1} \rightarrow G_{2}$. Since it is a subgroup of $G_{1} \times G_{2}$, it must be closed (since it is dense open in its closure). As in (a) we see it must be irreducible. Thus $h$ is an isomorphism of manifolds.

(c) Infinite definable fields are automatically connected ([Ma]), additively and multiplicatively.

Now let $F$ be a definable field. Applying 5.11 to the additive group structure of $F$, we may take $F$ to have a manifold structure, so that the graph of addition is closed.

Lemma 5.13. Let $F$ be a manifold carrying a definable field structure. Assume addition is a morphism from $F^{2}$ to $F$. Then so is multiplication.

Proof. There exists a dense open subset $U$ of $F^{3}$, and a closed subset $M$ of $F^{3}$, such that $M$ agrees on $U$ with the graph of multiplication. (Any definable subset has this property.) We may choose $M$ irreducible; in particular no component of $M$ is contained in the complement of $U$. So it is not the case that for generic $(x, y) \in F^{2}$ there exists $z$ with $(x, y, z) \in M$ and $(x, y, z) \notin U$. Thus for generic $(x, y) \in F^{2}$ there exists a unique $z$ such that $(x, y, z) \in M$, namely $z=x y$. Let $U_{2}$ be an open subset of $F^{2}$ such that if $(x, y) \in U_{2}$, then $(x, y, x y) \in M$, and there is no $z \neq x y$ with $(x, y, z) \in M$. Since $M \subseteq F^{3}$ is closed, and the graph of multiplication on $U_{2} \times F$ coincides with $M$, multiplication is a morphism $U_{2} \rightarrow F$.

Given $c=\left(c_{1}, c_{2}\right) \in F^{2}$, let $U_{2}-c=\left\{(x, y) \in F^{2}:\left(x+c_{1}, y+c_{2}\right) \in U_{2}\right\}$. Note that $F^{2}$ is covered by finitely many sets $U_{2}-c$ (in fact by $2 \operatorname{dim}(F)+1$ such sets). 
Claim. For any $c \in F,\{(x, y): c x=y\}$ is closed.

Proof. A definable subgroup of $F \times F$ is automatically closed, since it is dense open in its closure.

Hence multiplication by a generic element is a morphism. Let $c=\left(c_{1}, c_{2}\right) \in F^{2}$. Let $f:\left(U_{2}-c\right) \rightarrow F$ be defined by $f(x, y)=\left(x+c_{1}\right)\left(y+c_{2}\right) . f$ is the composition of the map $(x, y) \rightarrow\left(x+c_{1}, y+c_{2}\right)$ with the multiplication map $U_{2} \rightarrow F$. Both these maps are morphisms; hence by 5.10 , so is $f$.

Let $a, b \in F$ be arbitrary. Then $a b=\left(a+c_{1}\right)\left(b+c_{2}\right)-c_{1} b-c_{2} a-c_{1} c_{2}=$ $f(a, b)-c_{1} b-c_{2} a-c_{1} c_{2}$. Using $5.9,5.10$, we see that multiplication is a morphism on $(U-c)$. Since morphismhood is local, multiplication is a morphism.

Definition. $a \rightarrow a^{\prime}$ is a regular specialization if the point $a^{\prime}$ is regular on the locus of $a$.

A good specialization is defined recursively as follows. Regular specializations are good. Let $a=\left(a_{1}, a_{2}, a_{3}\right), a^{\prime}=\left(a_{1}^{\prime}, a_{2}^{\prime}, a_{3}^{\prime}\right), a \rightarrow a^{\prime}$. Suppose:

(i) $\left(a_{1}, a_{2}\right) \rightarrow\left(a_{1}^{\prime}, a_{2}^{\prime}\right)$ is good.

(ii) $a_{1} \rightarrow a_{1}^{\prime}$ is an isomorphism.

(iii) $a_{3} \in \operatorname{acl}\left(a_{1}\right)$.

Then $a \rightarrow a^{\prime}$ is good.

Lemma 5.14. Let $a \rightarrow a^{\prime}$ be a good specialization of rank $\leq 1$. Then any specializations $a b \rightarrow a^{\prime} b^{\prime}$, ac $\rightarrow a^{\prime} c^{\prime}$ can be amalgamated: there exist $b^{*}, c^{*}$ independent over $a, \operatorname{tp}\left(b^{*} / a\right)=\operatorname{tp}(b / a), \operatorname{tp}\left(c^{*} / a\right)=\operatorname{tp}(c / a), a b^{*} c^{*} \rightarrow a^{\prime} b^{\prime} c^{\prime}$.

Proof. If $a \rightarrow a^{\prime}$ is regular this is immediate from 4.7: Let $D_{1}$ be the set of regular points on the locus of $a$; since $a \rightarrow a^{\prime}$ is regular, $a^{\prime} \in D_{1}$. The dimension theorem is valid in $D_{1}^{2} \times D^{m}$, by 5.4 , and $a^{\prime} \notin E\left(C, D_{1}\right)$ by 4.13 . Let $D_{2}$ be the locus of $b$, and $C$ the locus of $(a, b)$ in $D_{1} \times D_{2}$. By 4.7, one can find $b^{*}$ in $C(a)$ with $\operatorname{rk}\left(b^{*} / a c\right)=\operatorname{dim} C(a)$, and in particular $\operatorname{rk}\left(b^{*} / a\right)=\operatorname{rk}(b / a)$, and $b^{*}$ is independent from $c$ over $a$, such that $a b^{*} c \rightarrow a^{\prime} b^{\prime} c^{\prime}$. Let $c^{*}=c$. Since $C(a)$ is $a$-irreducible, $\operatorname{tp}\left(b^{*} / a\right)=\operatorname{tp}(b / a)$.

Now suppose $a \rightarrow a^{\prime}$ is as in the definition of "good", and the lemma holds for $\left(a_{1}, a_{2}\right) \rightarrow\left(a_{1}^{\prime}, a_{2}^{\prime}\right)$. Amalgamating over $\left(a_{1}, a_{2}\right) \rightarrow\left(a_{1}^{\prime}, a_{2}^{\prime}\right)$, there exist $b^{*}, a_{3}^{*}$ such that $\operatorname{tp}\left(b^{*} a_{3}^{*} / a_{1} a_{2}\right)=\operatorname{tp}\left(b a_{3} / a_{1} a_{2}\right), b^{*} a_{3}^{*}$ is independent from $c$ over $a_{1} a_{2}$, and $a_{1} a_{2} a_{3}^{*} b^{*} a_{3} c \rightarrow a_{1}^{\prime} a_{2}^{\prime} a_{3}^{\prime} b^{\prime} a_{3}^{\prime} c^{\prime}$. Now $\left(a_{1} a_{3} a_{3}^{*}\right) \rightarrow\left(a_{1}^{\prime} a_{3}^{\prime} a_{3}^{\prime}\right)$ is a rank-0 specialization, by (ii) and (iii), so it is an isomorphism. Thus $a_{3}=a_{3}^{*}$, so $a b^{*} c \rightarrow a^{\prime} b^{\prime} c^{\prime}$ as required.

Lemma 5.15. Let $\left(a_{i}: i \in I\right)$ be independent over $b$ and indiscernible over $b, I$ infinite. Suppose $\left(a_{i}^{\prime}: i \in I\right)$ is indiscernible over $b^{\prime}$, and $a_{i} b \rightarrow a_{i}^{\prime} b^{\prime}$ for each $i$. Further suppose $\operatorname{rk}\left(b \rightarrow b^{\prime}\right) \leq 1$ and $b \rightarrow b^{\prime}$ is good. Then $\left(b a_{i}: i \in I\right) \rightarrow\left(b^{\prime} a_{i}^{\prime}: i \in\right.$ $I)$.

Proof. By 5.14 and induction, there exist $a_{i}^{*}$ independent over $b, \operatorname{tp}\left(a_{i}^{*} / b\right)=$ $\operatorname{tp}\left(a_{i} / b\right)$, such that $\left(b, a_{1}^{*}, a_{2}^{*}, \ldots\right) \rightarrow\left(b^{\prime}, a_{1}^{\prime}, a_{2}^{\prime}, \ldots\right)$. There are only infinitely many types over $\operatorname{acl}(b)$ extending $\operatorname{tp}\left(a_{i} / b\right)$; hence for some infinite $I,\left(b, a_{i}^{*}\right)_{i \in I}$ is indiscernible over $b$, and so has the same type as $\left(b, a_{i}\right)_{i \in I}$. Thus $\left(b, a_{i}\right)_{i \in I} \rightarrow\left(b^{\prime}, a_{i}^{\prime}\right)_{i \in I}$, and the lemma follows using indiscernibility. 


\section{INTERPRETING THE FIELD}

6.1. In this section we will interpret a field $F$ in the Zarisk structure $D$. We will work in the category of definable sets and maps, rather than the finer category of closed sets; the Zariski structure will enter only through the notion of a specialization. We first describe the proof in general terms.

Suppose first $D$ is in fact the affine line over an algebraically closed field $K$, and consider the task of recognizing the multiplicative group, given the Zariski structure. Let $F$ be a family of curves on $D^{2}$, all passing through the point $p=(0,0)$. Assume each curve $C$ in $F$ is the graph of a function, smooth at $(0,0)$. Then we may view $C_{i} \in F$ as coding the slope $\sigma_{i}$ of $C_{i}$ at $p$. The composition $C_{1} \circ C_{2}^{-1}$ will have slope $\sigma_{1} / \sigma_{2}$. Thus in this case we will interpret a copy of the multiplicative group if we could find the relation of tangency of two curves at a point $p$. This can be done as follows: we say two curves $C_{1}, C_{2}$ in a family are tangent at $p$ if there exist generic curves $C_{i}^{*}$ in the family meeting at two distinct points $q, q^{\prime}$, and a specialization carrying $C_{i}^{*}$ to $C_{i}$ and taking both $q$ and $q^{\prime}$ to the point $p$.

The idea above can in fact be carried out, even if the curves $C_{i}$ are not the graphs of rational functions. As observed by Evgenia Rabinovich, the group obtained in this way is not always the multiplicative group; the reason is that all our curves may have the same slope at $q_{0}$, in which case our process will discern the second (or higher) derivative. We do not concern ourselves with the nature of the group, and write it additively, as $(A,+, 0)$. (The group operation will be differentiated again, and the result then will be the additive group at all events.)

Next suppose $D$ is a curve over an algebraically closed field $K$, but not necessarily rational. Then there exists a finite-to-finite correspondence $\alpha$ between $D$ and $K$. We find a family $F$ of curves on $D^{2}$, all passing through a point $p_{0} \in D^{2}$. Via the correspondence $\alpha, F$ gives rise to a family $F^{\prime}$ of curves on $K^{2}$, passing through the point $q_{0} ; C$ corresponds to any component $C^{\prime}$ of the curve $\alpha \circ C \circ \alpha^{-1}$. This correspondence is also finite-to-finite. We are thus presented with the task of recognizing a group structure transformed by a finite-to-finite correspondence. The machinery for this is already available, and will be explained in $§ 6.2$.

Once we have an Abelian group $H$, we can repeat the process, with more information at our disposal. Given a family of functions $H \rightarrow H$, we can both add them (pointwise) and compose them. The two operations together, viewed as "acting on the tangent space" as above will give a field structure.

Certain technical difficulties present themselves when the above is carried out; in general it is not clear why the notion of tangency as defined above will not be a degenerate notion (e.g. $C_{1} \circ C_{2}^{-1}$ is tangent to $C_{3} \circ C_{4}^{-1}$ iff two of the four curves are equal). We show this is not the case by exploiting certain symmetries of the situation, and showing in effect that any such degeneracy would introduce an asymmetry. The need to keep track of these symmetries led us to a more technical presentation than the above sketch suggests.

6.2. We now describe the means of recognizing a group structure across a finiteto-finite correspondence. Suppose $G$ is a group acting on a set $X$ transitively, and let there be given finite-to-finite correspondences $\alpha_{1} \subseteq G \times G^{\prime}, \alpha_{2} \subseteq X \times X^{\prime}$, $\alpha_{3} \subseteq X \times X^{\prime \prime}$. Let $M=\{(g, x, y): g x=y\}$ be the graph of the group action, and let $M^{\prime} \subseteq G^{\prime} \times X^{\prime} \times X^{\prime \prime}$ be its transform, i.e., any component of $\left\{\left(g^{\prime}, x^{\prime}, y^{\prime}\right) \in\right.$ $G^{\prime} \times X^{\prime} \times X^{\prime \prime}$ : for some $\left.(g, x, y) \in M,\left(g, g^{\prime}\right) \in \alpha_{1},\left(x, x^{\prime}\right) \in \alpha_{2},\left(y, y^{\prime}\right) \in \alpha_{3}\right\}$. Then $M^{\prime}$ is called a pseudo-action. Note that any purely dependence-theoretic statement 
true of $M$ will also be true of $M^{\prime}$. It turns out that the simplest such statement suffices to determine $M^{\prime}$ as a pseudo-action. The following result is from [Hr5]; see also $[\mathrm{EH}]$ and $[\mathrm{B}]$.

For the rest of this section we assume $D$ is a strongly minimal set, or just a minimal set within some saturated structure, in which $\operatorname{dcl}(\emptyset)=\operatorname{acl}(\emptyset)$ is infinite. In other words for every definable subset $H$ of the ambient structure, with parameters, $H \cap D$ or $D-H$ are finite. (The strongly minimal case will suffice for the proofs of the theorems stated in this paper, but no further work is involved in making this weaker assumption. In some of the references, stability of the ambient structure is assumed, and the reader may feel more comfortable in this intermediate environment.)

Unless otherwise stated all elements $a, b, c$, etc. are elements of $D^{n}$ for some $n$.

Theorem 6.1. Let $\left(b_{1}, a_{2}, a_{3}\right)$ be points of $D^{\mathrm{eq}}, M=\operatorname{tp}\left(b_{1} a_{2} a_{3}\right)$. Then $M$ is a pseudo-action iff there exist $b_{2}, b_{3}, a_{1}$ such that for $\{i, j, k\}=\{1,2,3\}$ :

(i) $b_{i}, a_{j}$ are independent; $a_{k} \in \operatorname{acl}\left(b_{i}, a_{j}\right)$;

(ii) $b_{i}, b_{j}$ are independent; $b_{k} \in \operatorname{acl}\left(b_{i}, b_{j}\right)$;

(iii) $a_{i}$ is independent from $\left\{b_{1}, b_{2}, b_{3}\right\}$.

Moreover, if $b_{2}^{\prime} \in \operatorname{acl}\left(b_{2}\right), a_{3} \in \operatorname{acl}\left(b_{2}^{\prime}, a_{1}\right)$, then $b_{2} \in \operatorname{acl}\left(b_{2}^{\prime}\right)$.

This theorem will be used in both stages of the argument. First it will be applied to find a one-dimensional group, via its regular action. Then we will want to use it to find the field structure, via the group of affine transformations.

We now formulate a version more suitable for our needs.

Definition. $f=\left(f_{i j}: i \in I, j \in J\right)$ is an indiscernible array if whenever $i_{1}<$ $\cdots<i_{n}, j_{1}<\cdots<j_{m}$ then $\operatorname{tp}\left(f_{i_{\nu} j_{\mu}}: 1 \leq \nu \leq n, 1 \leq \mu \leq m\right)$ depends only on $n, m$. If at least the rank $r$ of this type depends only on $n, m$, we say that $\operatorname{rk}(f ; n, m)$ is defined and equals $r$. If $\operatorname{rk}(f ; n, m)$ is defined for all $n, m$, we say that $f$ is rank-indiscernible, of type $\alpha$, where $\alpha(n, m)=\operatorname{rk}(f ; n, m)$.

Remark 6.2. It is easy to see that if $f$ is rank-indiscernible, of rank $\alpha$, then there exists a polynomial $p \in Z[x, y]$ such that $\alpha(m, n)=p(m, n)$ for large $m, n . \quad p$ is either equal to $x y$ or is affine. If one considers indiscernible "arrays" of higher dimensions $d$, the polynomial can have degree up to $d$. It would be nice to determine the possibilities.

Lemma 6.3. Let $\left(f_{i j}: i, j<\omega\right)$ be an indiscernible array of elements of $D$, of type $\alpha$.

(a) Suppose $\alpha(n, m)=m+n-1$. Then there exists a definable one-dimensional group $(A,+)$ and elements $a_{i}, a^{j}$ of $A$ such that $\operatorname{acl}\left(f_{i j}\right)=\operatorname{acl}\left(a_{i}+a^{j}\right)$.

(b) Suppose $\alpha(m, n)=2 m+n-2(m \geq 2, n \geq 2)$. Then there exist a definable field $F$ and independent elements $a_{i}, b_{i}, c_{j} \in F, \operatorname{acl}\left(f_{i j}\right)=\operatorname{acl}\left(a_{i} c_{j}+b_{i}\right)$.

(c) Suppose $\alpha(m, n)=\lambda m+n-\lambda(m \geq 2, n \geq \lambda)$. Then there exist a definable group $G$ of dimension $\lambda$, a definable one-dimensional set $X$, a definable action of $G$ on $X$, and independent elements $g_{i}$ of $G$ and $a^{j}$ of $X$ such that $\operatorname{acl}\left(f_{i j}\right)=\operatorname{acl}\left(g_{i} \cdot a^{j}\right)$. (Moreover, $\lambda \leq 3$.)

Remark 6.4. We will only use the existence of a definable one-dimensional group, or field, and not the full conclusion. In case (a) the proof does not require an infinite array; it suffices to have a rank-indiscernible array, of size at least $3 \times 3$. This does not hold for 6.3(b) however. For example in a vector space over the rationals, let $a_{i}, b_{j}, c_{k}$ be linearly independent; then $f_{j j}=a_{i}+b_{j}+i c_{j}$ forms a rank-indiscernible 
array of eventual type $2 m+n-2$, but no field is interpretable in the vector space. (One could also get a finite indiscernible array of any prescribed size, though of course not with $\operatorname{acl}(\emptyset)=\operatorname{dcl}(\emptyset)$ infinite.)

Remark 6.5. An indiscernible array of the type mentioned in 6.3(b) can be viewed as a diagonal in a generalized three-dimensional array $\left(f_{i j k}: i, j, k<\omega\right)$, with type $\alpha(n, m, p)=n+m+p-2$ (eventually). Such a three-dimensional array can arise from a field $\left(f_{i j k}=a_{i}\left(c_{j}+b_{k}\right)\right)$ but also from an Abelian group structure $\left(f_{i j k}=a_{i}+b_{j}+c_{k}\right)$. Probably these are the only possibilities.

Proof of 6.3. We prove 6.3(c). The other clauses follow using [Hr6] or [Ch]. We will use the following general remark. The same proof is valid in any homogeneous geometry, which has been sufficiently localized.

Claim 1. Suppose $\operatorname{rk}\left(c / d_{1} d_{2}\right)=\operatorname{rk}\left(c / d_{1}\right)=\operatorname{rk}\left(c / d_{2}\right)$. Then there exists $e \in$ $\operatorname{acl}\left(d_{1}\right) \cap \operatorname{acl}\left(d_{2}\right)$ such that $\operatorname{rk}(c / e)=\operatorname{rk}\left(c / d_{1} d_{2}\right)$.

Proof. Let $E=\operatorname{acl}\left(d_{1}\right) \cap \operatorname{acl}\left(d_{2}\right)$. Let $c=\left(c_{1}, \ldots, c_{m}\right)$; say $c_{1}, \ldots, c_{k}$ are independent from $d_{1} d_{2}$, while $c \in \operatorname{acl}\left(c_{1} \cdots c_{k}, d_{1} d_{2}\right)$. Then

$$
c \in \operatorname{acl}\left(c_{1} \cdots c_{k}, d_{i}, E\right) \quad(i=1,2) .
$$

We must show that $c \in \operatorname{acl}\left(c_{1} \cdots c_{k}, E\right)$. We use induction on $\operatorname{rk}\left(d_{i} / E\right)$. If $\operatorname{rk}\left(d_{1} / E\right)$ $=\operatorname{rk}\left(d_{2} / E\right)=0$, we are done. Otherwise say $\operatorname{rk}\left(d_{2} / E\right)=r>0$, and choose $d \in \operatorname{acl}\left(d_{2}\right), d \in D, d \notin E$. By assumption $E$ is infinite. Now for all but finitely many $d^{\prime} \in E$, there exists $d_{2}^{\prime}$ such that:

(i) $\operatorname{rk}\left(d_{2}^{\prime} / d^{\prime} E\right)<r$,

(ii) $c \in \operatorname{acl}\left(c_{1} \cdots c_{k}, d_{2}^{\prime}, E\right)$.

(Since the corresponding facts are true of $d$, with witness $d_{2}$, and there exists a single formula ensuring this.) Choose such a $d^{\prime} \in E$. Further choose $d_{2}^{\prime}$ satisfying (i), (ii). We may choose $d_{2}^{\prime}$ independent from $d_{1}$ over $E \cup\left\{c, d_{2}\right\}$. Since $c$ is independent from $d_{1}$ over $E \cup\left\{d_{2}\right\}, d_{2}^{\prime}$ is independent from $d_{1}$ over $E \cup\left\{d_{2}\right\}$. Thus $\operatorname{acl}\left(d_{2}^{\prime} E\right) \cap \operatorname{acl}\left(d_{1} E\right) \subseteq \operatorname{acl}\left(d_{2} E\right) \cap \operatorname{acl}\left(d_{1} E\right)=E$. Using (i) and induction we have $c \in \operatorname{acl}\left(c_{1} \cdots c_{k}, E\right)$, as required.

We will now find a configuration $\left(a_{1}, a_{2}, a_{3}, b_{1}, b_{2}, b_{3}\right)$ as in 6.1. We may extend the given array $\left(f_{i j}\right)$ so as to be defined for all integers $i, j$ (perhaps in some elementary extension). Let:

$$
\begin{gathered}
b_{1}=\left(f_{1,-c}, \ldots, f_{1,-1}\right), \quad b_{3}=\left(f_{2,-c}, \ldots, f_{2,-1}\right), \\
a_{3}=f_{1,0}, \quad a_{1}=f_{2,0} .
\end{gathered}
$$

We need to define $b_{2}$ and $a_{2}$. This is done as follows.

Claim 2. (a) There exists $a_{2} \in \operatorname{acl}\left(b_{1} a_{3}\right) \cap \operatorname{acl}\left(b_{3} a_{1}\right)$ with $\operatorname{rk}\left(a_{2}\right)=1$.

(b) There exists $b_{2} \in \operatorname{acl}\left(b_{1} b_{3}\right)$ with $\operatorname{rk}\left(b_{2}\right) \leq \lambda, \operatorname{rk}\left(a_{1} a_{3} / b_{2}\right)=1$.

Proof. (a) Let $y=\left(f_{3,-c}, \ldots, f_{3,0}\right)$. One computes from the given type $\alpha$ of the array that $\operatorname{rk}\left(y / b_{1} a_{3}\right)=\operatorname{rk}\left(y / b_{2} a_{1}\right)=\operatorname{rk}\left(y / b_{1} a_{3} b_{2} a_{1}\right)=\lambda$. By Claim 1 , there exists $a_{2} \in \operatorname{acl}\left(b_{1} a_{3}\right) \cap \operatorname{acl}\left(b_{2} a_{1}\right)$ such that $\operatorname{rk}\left(y / a_{2}\right)=\lambda$. We have $\operatorname{rk}\left(a_{2}\right) \geq 1 \operatorname{since}$ $\operatorname{rk}(y)=\lambda+1$. Now

$$
\begin{aligned}
2 \lambda+1-\operatorname{rk}\left(a_{2}\right) & =\operatorname{rk}\left(b_{1} a_{3} b_{2} a_{1} / a_{2}\right) \leq \operatorname{rk}\left(b_{1} a_{3} / a_{2}\right)+\operatorname{rk}\left(b_{2} a_{1} / a_{2}\right) \\
& =\operatorname{rk}\left(b_{1} a_{3}\right)+\operatorname{rk}\left(b_{2} a_{1}\right)-2 \operatorname{rk}\left(a_{2}\right)=2(\lambda+1)-2 \operatorname{rk}\left(a_{2}\right) .
\end{aligned}
$$

So $\operatorname{rk}\left(a_{2}\right) \leq 1$ 
(b) Let $b_{1}^{\prime}=\left(f_{1,-2 c}, \ldots, f_{1,-c-1}\right), b_{3}^{\prime}=\left(f_{2,-2 c}, \ldots, f_{2,-c-1}\right)$. By the type of the array, $\operatorname{rk}\left(a_{1} a_{3} / b_{1} b_{3}\right)=\operatorname{rk}\left(a_{1} a_{3} / b_{1}^{\prime} b_{3}^{\prime}\right)=\operatorname{rk}\left(a_{1} a_{3} / b_{1} b_{3} b_{1}^{\prime} b_{3}^{\prime}\right)=1$. By Claim 1, there exists $b_{2} \in \operatorname{acl}\left(b_{1} b_{3}\right) \cap \operatorname{acl}\left(b_{1}^{\prime} b_{3}^{\prime}\right)$ with $\operatorname{rk}\left(a_{1} a_{3} / b_{2}\right)=1$. As in (a), we have:

$$
\begin{aligned}
3 \lambda-\operatorname{rk}\left(b_{2}\right) & =\operatorname{rk}\left(b_{1} b_{3} b_{1}^{\prime} b_{3}^{\prime} / b_{2}\right) \leq \operatorname{rk}\left(b_{1} b_{3}\right)+\operatorname{rk}\left(b_{1}^{\prime} b_{3}^{\prime}\right)-2 \operatorname{rk}\left(b_{2}\right) \\
& =2 \lambda+2 \lambda-2 \operatorname{rk}\left(b_{2}\right) .
\end{aligned}
$$

So $\operatorname{rk}\left(b_{2}\right) \leq \lambda$.

By choosing $b_{2}$ in (b) with $\operatorname{rk}\left(b_{2}\right)$ least possible, we may satisfy the "moreover" clause in 6.1 . The problem is thus only to show that necessarily $\operatorname{rk}\left(b_{2}\right)=\lambda$. Since $b_{2} \in \operatorname{acl}\left(b_{1} b_{3}\right)$, and using the indiscernibility, we have that

$$
\operatorname{rk}\left(f_{1, n} f_{2, n} / b_{2}\right)=1 \quad \text { for all } n \text {. }
$$

Thus $\operatorname{rk}\left(f_{11} \cdots f_{1, \lambda}, f_{21} \cdots f_{2, \lambda} / b_{2}\right) \leq \lambda$, and $\operatorname{so} \operatorname{rk}\left(f_{11} \cdots f_{1, \lambda}, f_{21} \cdots f_{2, \lambda}\right)<2 \lambda$. This contradicts the formula $\alpha(2, \lambda)=2 \lambda+\lambda-\lambda=2 \lambda$.

In order to show that $b_{i} \in \operatorname{acl}\left(b_{j}, b_{k}\right)$ for any choice of $i, j, k$ it suffices now to show that $b_{3}, b_{1}$ are independent (and $b_{3}, b_{2}$ are independent). This follows from the fact that $b_{3} \in \operatorname{acl}\left(b_{1}^{\prime} b_{2}^{\prime}\right)$, once we know that $b_{1}, b_{1}^{\prime}, b_{2}^{\prime}$ are independent. We know that a $2 \times \lambda$ rectangle from $\left(f_{i j}\right)$ is independent; we need to know that $f_{1 n}$ is independent from $f_{1,<n}, f_{2,<n}$. If this were not so, then by indiscernibility, $f_{1 N} \in \operatorname{acl}\left(f_{1,<n}, f_{2,<n}\right)$ and $f_{2 N} \in \operatorname{acl}\left(f_{1,<n}, f_{2,<n}\right)$ for $N \geq n$, so that $\alpha(2, N)$ is bounded, a contradiction.

6.3. Finding the indiscernible arrays. From now on we assume that $D$ is a strongly minimal set; or even that $D$ is an infinitely-definable set in some saturated structure, such that every definable subset of $D$ is finite or cofinite. We also assume the existence of the notion of a specialization between tuples of elements of $D^{\mathrm{eq}}$, and the notion of a regular specialization. We collect the properties of these notions that we will use.

Assumptions. We adopt the notations 4.5, 4.6, and the definition 5.14(a) of "good". (Formally, we may define specializations on nonspecial sorts by fixing a representation of such a sort as a disjoint union of special sorts, and using the induced topology; in fact of course we will only use elements of special sorts.)

6.6. (1) If $\operatorname{tp}(a)=\operatorname{tp}\left(a^{\prime}\right)$, then $a \rightarrow a^{\prime}$. If $a \rightarrow a^{\prime}$ and $a^{\prime} \rightarrow a^{\prime \prime}$, then $a \rightarrow a^{\prime \prime}$. Let $a=\left(a_{i}: i \in I\right), \iota: J \rightarrow I$ a map of index sets, $a \iota=\left(a_{\iota(j)}: j \in J\right)$. If $a \rightarrow a^{\prime}$, then $a \iota \rightarrow a^{\prime} \iota$. If $a_{1}=a_{2}$ and $a \rightarrow a^{\prime}$, then $a_{1}=a_{1}^{\prime}$. If $a \in D$ is a generic singleton and $a^{\prime} \in D$, then $a \rightarrow a^{\prime}$.

(2) If $a \rightarrow a^{\prime}$, then $\operatorname{tp}(a)=\operatorname{tp}\left(a^{\prime}\right)$, or $\operatorname{rk}(a)>\operatorname{rk}\left(a^{\prime}\right)$.

(3) (Dimension theorem) Let $a \rightarrow a^{\prime \prime}$; suppose $a(1), a(2) \in D, a(1) \neq a(2)$, $a^{\prime \prime}(1)=a^{\prime \prime}(2)$. Then there exists $a^{\prime}, a \rightarrow a^{\prime} \rightarrow a^{\prime \prime}, a^{\prime}(1)=a^{\prime}(2)$, with $\operatorname{rk}\left(a \rightarrow a^{\prime}\right)=1$.

(4) Let $\left(a_{i}: i \in I\right)$ be independent over $b$ and indiscernible over $b$. Suppose $\left(a_{i}^{\prime}: i \in I\right)$ is indiscernible over $b^{\prime}$, and $a_{i} b \rightarrow a_{i} b^{\prime}$ for each $i$. Further suppose $\operatorname{rk}\left(b \rightarrow b^{\prime}\right) \leq 1$, and $b \rightarrow b^{\prime}$ is good. Then $\left(b a_{i}: i \in I\right) \rightarrow\left(b^{\prime} a_{i}^{\prime}: i \in I\right)$. (5.15).

(5) Amalgamation Lemma 5.14.

(6) Let $H$ be a definable (one-dimensional) Abelian group. Then if $\sum a_{i}=0$ in $H$ and $\left(a_{i}\right) \rightarrow\left(a_{i}^{\prime}\right)$ and each $a_{i}^{\prime}$ is a generic element of $H$, then $\sum a_{i}^{\prime}=0$. In particular if $a+b=c+d,(a b c d) \rightarrow\left(a^{\prime} b^{\prime} c^{\prime} d^{\prime}\right)$ and $a^{\prime}, \ldots, d^{\prime}$ are generic elements of $H$, then $a^{\prime}=c^{\prime}$ implies $b^{\prime}=d^{\prime}$. 
(7) If $a a^{\prime} \rightarrow b b^{\prime}$, if $a \rightarrow b, a^{\prime} \rightarrow b^{\prime}$ are regular specializations, and if $a, a^{\prime}$ are independent, then $a a^{\prime} \rightarrow b b^{\prime}$ is regular. If $a$ is a generic element of $D$, or of a one-dimensional definable group, then $a \rightarrow a^{\prime}$ is always regular. Isomorphisms are regular.

We will use below the following remark on existence of indiscernible arrays (valid in saturated models of stable theories). If $\left(c_{i j}: i \in I, j \in J\right)$ is an array and $I^{\prime}, J^{\prime}$ are subsets of $I, J$, we write $c_{I^{\prime} J^{\prime}}$ for $\left(c_{i j}: i \in I^{\prime}, j \in J^{\prime}\right)$. If $\left|I^{\prime}\right|=m,\left|J^{\prime}\right|=n$ we call $c_{I^{\prime} J^{\prime}}$ an $m \times n$ rectangle from $c_{i j}(m \times m$ square, if $m=n)$.

Lemma 6.7. Let $\left(c_{i j}: i \in I, j \in J\right)$ be an array, with infinite index sets $I, J$. Suppose $P_{m n}$ is a partial type, and each $m \times n$ rectangle from $\left(c_{i j}\right)$ satisfies $P_{m n}$. Then there exists an indiscernible array $\left(d_{i j}: i, j=1,2,3, \ldots\right)$ such that $P_{m n}$ holds for each $m \times n$ rectangle from $\left(d_{i j}\right)$.

Proof. The usual method of finding indiscernibles (see [CK]) shows that one may assume: $I=J=\{1,2, \ldots\}$, and $\left(c_{I j}: j \in J\right)$ is indiscernible (i.e., $\left(c_{I^{\prime} j}: j \in J\right)$ is indiscernible for any finite $I^{\prime} \subseteq I$ ). By stability, it is indiscernible as a set. Similarly we may assume that $\left\{c_{i J}: i \in I\right\}$ is indiscernible. Let $I_{m}, J_{n}$ be two particular subsets of $I, J$ of size $m, n$. It follows that any $m \times n$ rectangle from $\left(c_{i j}\right)$ has the same type as a rectangle $\left(c_{I^{\prime} J_{m}}\right)$, and that $\left(c_{I^{\prime} J^{\prime}}\right)$ has the same type as $c_{I_{n} J_{m}}$. Thus $\left(c_{i j}\right)$ is now an indiscernible array.

Notation. We write $A \downarrow B \mid C$ to mean: $A, B$ are free over $C$. (Cf. $\S 3$.)

Lemma 6.8. Let $\left(A_{i j}: i \leq M, j \leq N\right)$ be an indiscernible array, $M, N \geq 2$. Assume $\operatorname{rk}(A ; 2,2)=4, \operatorname{rk}(A ; 2,1)=3$ and that if $C, C^{\prime}$ are two adjacent $2 \times 1$ rectangles, then $\operatorname{rk}\left(\operatorname{dcl}(C) \cap \operatorname{dcl}\left(C^{\prime}\right)\right)=2$.

Let $\left(A_{i j}: i, j\right) \rightarrow\left(a_{i j}: i, j\right)$ be a rank-1 specialization. Suppose $\operatorname{tp}\left(a_{i j} / \operatorname{acl}(\emptyset)\right)$ is constant with $i, j$ and $\operatorname{rk}(a ; 2,1)=2$. Also assume $A_{i j} A_{i^{\prime} j^{\prime}} \rightarrow a_{i^{\prime} j} a_{i^{\prime} j}$ is good for any $i, i^{\prime}, j$. Then a is rank-indiscernible, of type $m+n-1$.

Proof. Claim 1. Let $c_{i j}=a_{f(i) g(j)}(i, j=1,2)$. Assume

$$
\left(A_{i j}: i=1,2, j=1,2\right) \rightarrow\left(c_{i j}: i=1,2, j=1,2\right)
$$

is a rank-1 specialization, $\operatorname{tp}\left(c_{i j} / \operatorname{acl}(\emptyset)\right)=q, \operatorname{rk}(q)=1$. Then $\operatorname{rk}\left(c_{21} c_{22} / c_{11} c_{12}\right) \leq 1$.

Proof. Suppose for contradiction that

(i) $\operatorname{rk}\left(c_{21} c_{22} / c_{11} c_{12}\right)=2$.

In particular

(ii) $\operatorname{rk}\left(c_{11} c_{21}\right)=2, \operatorname{rk}\left(c_{12} c_{22}\right)=2$.

Thus $\operatorname{rk}\left(c_{12} c_{22} / c_{11} c_{21}\right)=1$. Introduce new elements $c_{10}$ and $c_{20}$ such that: $\operatorname{rk}\left(c_{10} c_{20} / c_{11} c_{12} c_{21} c_{22}\right)=1$ and $\operatorname{stp}\left(c_{11} c_{21} c_{10} c_{20}\right)=\operatorname{stp}\left(c_{21} c_{11} c_{22} c_{12}\right)$. This is possible since $\operatorname{stp}\left(c_{11} c_{21}\right)=\operatorname{stp}\left(c_{21} c_{11}\right)$. Thus $c_{21} c_{11} c_{22} c_{12} \rightarrow c_{11} c_{21} c_{10} c_{20}$. Now

$$
\operatorname{tp}\left(A_{11} A_{21} A_{12} A_{22}\right)=\operatorname{tp}\left(A_{21} A_{11} A_{22} A_{12}\right)
$$

so

$$
A_{11} A_{21} A_{12} A_{22} \rightarrow A_{21} A_{11} A_{22} A_{12} .
$$

Also we know that

$$
A_{21} A_{11} A_{22} A_{12} \rightarrow c_{21} c_{11} c_{22} c_{12} .
$$


Composing these three specializations, we get:

(iii) $A_{11} A_{21} A_{12} A_{22} \rightarrow c_{11} c_{21} c_{10} c_{20}$.

We also have:

(iv) $A_{11} A_{12} A_{12} A_{22} \rightarrow c_{11} c_{12} c_{12} c_{22}$.

By assumption, $A_{11} A_{12} \rightarrow c_{11} c_{12}$ is a good specialization, and by (ii) and the fact that $\operatorname{rk}(A ; 2,1)=3$, it has rank 1 . Thus by $6.6(5)$ (the amalgamation lemma for specializations 5.7), there exist $A_{10}, A_{20}$ with $\operatorname{rk}\left(A_{10} A_{20} / A_{11} A_{21} A_{12} A_{22}\right)=$ $\operatorname{rk}\left(A_{12} A_{22} / A_{11} A_{21}\right)$, and $\operatorname{tp}\left(A_{11} A_{20} / A_{11} A_{12}\right)=\operatorname{tp}\left(A_{12} A_{22} / A_{11} A_{21}\right)$, such that $\left(A_{i j}: i=1,2, j=0,1,2\right) \rightarrow\left(c_{i j}: i=1,2, j=0,1,2\right)$. In particular

(v) $\left(A_{i j}: i=1,2, j=0,2\right) \rightarrow\left(c_{i j}: i=1,2, j=0,2\right)$.

Now the assumptions on $A$ give:

$$
\operatorname{rk}\left(A_{12} A_{22} / A_{11} A_{21}\right)=1=\operatorname{rk}\left(A_{12} A_{22} / \operatorname{dcl}\left(A_{12} A_{22}\right) \cap \operatorname{dcl}\left(A_{11} A_{21}\right)\right) ;
$$

from this we calculate:

(vi) $\operatorname{rk}\left(A_{i j}: i=1,2, j=0,2\right)=4$.

Now from the choice of $c_{10} c_{20}$, we compute $\operatorname{rk}\left(c_{10} c_{20} / c_{12} c_{22}\right)$. We have

$$
\left\{c_{12} c_{22}\right\} \downarrow
$$

but by (i), $\operatorname{rk}\left(c_{21} / c_{11} c_{12} c_{22}\right)=1$, so

$$
\left\{c_{12} c_{22}\right\} \downarrow c_{21} \mid c_{11} .
$$

Thus by transitivity

$$
\left\{c_{12} c_{22}\right\} \downarrow\left\{c_{10} c_{20}\right\} \mid c_{11} .
$$

Now $\operatorname{rk}\left(c_{20} / c_{11}\right)=\operatorname{rk}\left(c_{12} / c_{21}\right)=1$, so

$$
\operatorname{rk}\left(c_{20} / c_{12} c_{22}\right)=1 \text {. }
$$

Also $\operatorname{rk}\left(c_{10} / c_{20} c_{11} c_{21}\right)=\operatorname{rk}\left(c_{22} / c_{12} c_{21} c_{11}\right)=1$ by (i). Hence

(vii) $\operatorname{rk}\left(c_{10} c_{20} c_{12} c_{22}\right)=4$.

Hence by (vi), the specialization (v) is an isomorphism. This is absurd since $\operatorname{rk}\left(c_{11} c_{21}\right) \leq 2$ while $\operatorname{rk}\left(A_{11} A_{21}\right)=3$.

Claim 2A. For any set $*$ of $i$-indices and $j \geq 2, \operatorname{rk}\left(a_{*, j} / a_{*,<j}\right) \leq 1$.

Proof. Otherwise, $\operatorname{rk}\left(a_{i j} a_{i^{\prime} j} / a_{i 1} a_{i^{\prime} 1}\right)=2$ for some $i \neq i^{\prime}$. Since $\operatorname{rk}(a ; 2,1)=$ $2, \operatorname{rk}\left(a_{i j} a_{i^{\prime} j} a_{i 1} a_{i^{\prime} 1}\right)=4=\operatorname{rk}(A ; 2,2)$, so $A_{i j} A_{i^{\prime} j} A_{i 1} A_{i^{\prime} 1} \rightarrow a_{i j} a_{i^{\prime} j} a_{i 1} a_{i^{\prime} 1}$ is an isomorphism, contradicting the fact that $\operatorname{rk}(A ; 2,1)=3$.

Claim 2B. For any set $*$ of $j$-indices and $i \geq 2, \operatorname{rk}\left(a_{i, *} / a_{<i, *}\right) \leq 1$.

Proof. Suppose otherwise. Then there exist $j_{1}<j_{2}$, with $\operatorname{rk}\left(a_{i j_{1}}, a_{i j_{2}} / a_{<i, *}\right)=2$. Let $c_{1 \nu}=a_{1 j_{\nu}}, c_{2 \nu}=a_{i j_{\nu}}(\nu=1,2)$. Since $\operatorname{rk}\left(c_{21} c_{22} / c_{11} c_{12}\right)=2$ and $\operatorname{rk}\left(c_{11}\right)=1$, we have $\operatorname{rk}\left(c_{i j}: i=1,2, j=1,2\right) \geq 3$. Thus $\left(A_{i j}: i=1,2, j=1,2\right) \rightarrow\left(c_{i j}: i=\right.$ $1,2, j=1,2)$ is a rank- 0 or rank- 1 specialization. The first case would mean that it is an isomorphism, giving a contradiction to $\operatorname{rk}\left(c_{11}\right)=1, \operatorname{rk}\left(A_{11}\right)>1$. The second contradicts Claim 1.

It follows immediately by induction on $n$ that any $m \times n$ rectangle from $a$ has rank $\leq m+n-1$. Suppose, for contradiction, that inequality is strict for some $m \times n$ rectangle. Then by Claim $2 \mathrm{~A}$ it remains so for the $m \times(n+1)$ rectangle obtained by adjoining an adjacent $m \times 1$ array; and similarly by Claim 2B, for the $(m+1) \times(n+1)$ rectangle obtained by further adjoining a $1 \times(n+1)$ array. Continuing in this way one finds that the inequality is strict for $m=M, n=N$, contradicting the assumption that the specialization $A \rightarrow a$ has rank 1 . 
Lemma 6.9. Let $A_{i j}$ be an indiscernible array with $\operatorname{rk}(A ; m, n)=2 m+n-1$. Suppose that if $C, C^{\prime}$ are two adjacent $1 \times 3$ rectangles, then

$$
\operatorname{rk}\left(\operatorname{dcl}(C) \cap \operatorname{dcl}\left(C^{\prime}\right)\right)=2 .
$$

Let $\left(A_{i j}: i \leq M, j \leq N\right) \rightarrow\left(a_{i j}: i \leq M, j \leq N\right)$ be a rank-1 specialization, and suppose $\operatorname{rk}(a ; 1, k)=k$. Further assume $A_{i j} A_{i j^{\prime}} A_{i j^{\prime \prime}} \rightarrow a_{i j} a_{i j^{\prime}} a_{i j^{\prime \prime}}$ is good for any $i, j, j^{\prime}, j^{\prime \prime}$. Then a is rank-indiscernible, of type $2 m+n-2(m \geq 2, n \geq 2)$.

Proof. Similar to the proof of 6.8. We first observe that any $m \times n$ rectangle from $a$ has rank $\leq 2 m+n-2$. Otherwise the specialization $A \rightarrow a$ would be an isomorphism on this rectangle and hence on each of its elements, but $\operatorname{rk}\left(A_{i j}\right)=2$, $\operatorname{rk}\left(a_{i j}\right)=1$.

Claim 1. Let $\left(c_{i j}\right)$ be a $2 \times 3$ rectangle from $a$. Assume $\left(A_{i j}: i=1,2, j=\right.$ $1,2,3) \rightarrow\left(c_{i j}: i=1,2, j=1,2,3\right)$ is a rank-1 specialization, $\operatorname{rk}(c ; 1, k)=k$. Then it is not the case that $\operatorname{rk}\left(c_{* 3} / c_{* 1} c_{* 2}\right)=2$.

Proof. Suppose $\operatorname{rk}\left(c_{* 3} / c_{* 1} c_{* 2}\right)=2$. Then $\operatorname{rk}\left(c_{* 1} c_{* 2}\right)=3$. Permuting the indices 1 and 2 if necessary, we may assume $\operatorname{rk}\left(c_{12} c_{21} c_{22}\right)=3$. Define $c_{0 *}$ with $\operatorname{rk}\left(c_{0 *} / c_{1 *} c_{2 *}\right)=2$ and $\operatorname{stp}\left(c_{0 *} c_{1 *}\right)=\operatorname{stp}\left(c_{2 \#} c_{1 \#}\right)$, where $*=(1,2,3), \#=(3,1,2)$. One obtains a contradiction as in the proof of Claim 1 of 6.8 .

Claim 2A. For any set $*$ of $j$-indices and $i \geq 2, \operatorname{rk}\left(a_{i, *} / a_{<i, *}\right) \leq 2$. This is indeed immediate from our assumptions, since if the claim fails we may take $*$ to be a set of three indices, and $\operatorname{since} \operatorname{rk}(a ; 1,3)=3$ by assumption, we would get a $2 \times 3$ rectangle of rank 6 , contrary to the initial observation.

Claim 2B. For any set $*$ of $i$-indices and $j \geq 3, \operatorname{rk}\left(a_{*, j} / a_{*,<j}\right) \leq 1$.

To prove $2 \mathrm{~B}$ note that we may take $*$ to consist to two indices, say 1 and 2 . Further by Claim 2A, we must have $\operatorname{rk}\left(a_{1 j} a_{2 j}: j \leq N\right) \geq 2+N$, since otherwise by induction on $m, \operatorname{rk}\left(a_{i j}: j \leq N, i \leq m\right)<2 m+N-2$, contradicting the assumption with $M=m$ that $A \rightarrow a$ is a rank-1 specialization. We next observe that as a consequence of Claim 1, there cannot be two distinct values of $j$ for which the present claim fails. Hence if the claim fails at $j_{0}$, then $2+N \leq \operatorname{rk}\left(a_{* j}: j \leq N\right) \leq$ $\operatorname{rk}\left(a_{* j}: j \leq j_{0}\right)+\left(N-j_{0}\right)$ so $\operatorname{rk}\left(a_{* j}: j \leq j_{0}\right) \geq 2+j_{0}$ and hence $\operatorname{rk}\left(a_{* j}: j<j_{0}\right) \geq j_{0}$. From this and the fact that the claim holds below $j_{0}$ it follows that $\operatorname{rk}\left(a_{* 1}\right)=2$ and that for some $j^{\prime}<j_{0}, \operatorname{rk}\left(a_{* 1} a_{* j^{\prime}}\right)=3$. This again contradicts Claim 1 .

Now the proof is finished as in 6.8.

Lemma 6.10. If $D$ is nondegenerate, then there exists a definable one-dimensional Abelian group.

Proof. We may assume $D$ is not locally modular, otherwise the conclusion holds by [Hr2]. So there exists a two-dimensional family $C(e) \subseteq D^{2}(e \in E)$ of "plane curves". We may assume the family is normal (3.9). Write $C(e, a, b)$ for " $(a, b) \in$ $C(e)$ ". Write $C^{2}\left(e ; a b, a^{\prime} b^{\prime}\right)$ if $C(e ; a, b)$ and $C\left(e ; a^{\prime} b^{\prime}\right)$, and if $a b, a^{\prime} b^{\prime}$ are independent over $e$ and have the same type over $\operatorname{acl}(e)$, and $e$ is a generic point of $E$. Then

(i) $C^{2}\left(e ; a b, a^{\prime} b^{\prime}\right)$ implies that $\operatorname{rk}\left(a b a^{\prime} b^{\prime}\right)=4$.

(ii) $C^{2}\left(e ; a b, a^{\prime} b^{\prime}\right)$ implies $e a b a^{\prime} b^{\prime} \rightarrow e a b a b$.

The first statement follows from the following computations: $\operatorname{rk}\left(e / a b a^{\prime} b^{\prime}\right)<$ $\operatorname{rk}(e / a b)$

$<\operatorname{rk}(e)=2 \operatorname{so} \operatorname{rk}\left(e / a b a^{\prime} b^{\prime}\right)=0 ;$ and $\operatorname{so~} \operatorname{rk}\left(a b a^{\prime} b^{\prime}\right)=\operatorname{rk}(e)+\operatorname{rk}(a b / e)+\operatorname{rk}\left(a^{\prime} b^{\prime} / e\right)$ $=2+1+1=4$.

The second statement follows from 6.6(4). Fix an integer $M$ momentarily. Let $a_{i}, b_{j}(i, j=0,1,2, \ldots, M)$ be independent generic elements of $D$. Then for 
$i, j \geq 1$ there exists $e_{i j} \in E$ such that $C^{2}\left(e_{i j} ; a_{i} b_{j}, a_{0} b_{0}\right)$. Let $A_{i j}=\left(a_{i}, b_{j}, e_{i j}\right)$, $A=\left(A_{i j}\right)_{i j \geq 1}$. We may choose $e_{i j}$ so that $\left(A_{i j}: i, j \geq 0\right)$ is indiscernible (first make arbitrary choices, then use 6.7). Note that $e_{i j} \in \operatorname{acl}\left(a_{0} b_{0} a_{i} b_{j}\right)$, and so the assumptions on $A$ in 6.8 are valid over the base $\left\{a_{0} b_{0}\right\}$. Write $x \rightarrow * y$ for $\left(x, a_{0}, b_{0}\right) \rightarrow\left(y, a_{0}, b_{0}\right)$.

Claim 1. Let $A_{i j}^{\prime}=A_{i 1}$ for $j \geq 1$. Then $A \rightarrow * A^{\prime}$.

Proof. Immediate from 6.6(4).

Claim 2. Let $A_{i j}^{\prime \prime}=\left(a_{0}, b_{0}, e_{i 1}\right)$. Then $A^{\prime} \rightarrow * A^{\prime \prime}$.

Proof. $A_{i j}^{\prime \prime}$ and $A_{i j}^{\prime}$ do not depend on $j$, so as specializations respect repeated entries, it suffices to show that $\left(A_{i 1}^{\prime}: i\right) \rightarrow *\left(A_{i 1}^{\prime \prime}: i\right)$.

Again this follows from 6.6(4): $a_{0} b_{0} b_{1} \rightarrow a_{0} b_{0} b_{0}$ by (ii) and this is a good rank1 specialization (since $\left(a_{0} b_{0} b_{1}\right)$ is a generic point of $\left.D^{3}\right) . A_{i 1}^{\prime}$ is indiscernible and independent over $a_{0} b_{0} b_{1}$, and $A_{i 1}^{\prime \prime}$ is indiscernible over $a_{0} b_{0} b_{0}$. For any given $i$, $\left(a_{i}, b_{1}, e_{i 1}, a_{0}, b_{0}\right) \rightarrow\left(a_{0}, b_{0}, e_{i 1}, a_{0}, b_{0}\right)$ by (ii). Thus $6.6(4)$ applies.

Claim 3. There exists $A^{*}=\left(A_{i j}^{*}\right)$ and a rank-1 specialization $A \rightarrow * A^{*}$, such that:

(i) $\operatorname{stp}\left(A_{i j}^{*} / a_{0} b_{0}\right)$ does not depend on $i, j$, and has rank 1 .

(ii) $A_{i j}^{*}=\left(a_{0} b_{0} e_{i j}^{*}\right)$ for some $e_{i j}^{*}$.

(iii) $A^{*}$ is rank indiscernible, of type $m+n-1$.

By the two claims, $A \rightarrow * A^{\prime \prime}$. By 6.6(3) there exists $A^{*}=\left(A_{i j}^{*}\right), A_{i j}^{*}=$ $\left(a_{i}^{*}, b_{i}^{*}, e_{i j}^{*}\right)$, such that $A \rightarrow * A^{*} \rightarrow * A^{\prime \prime}, a_{1}^{*}=a_{0}$ and $A \rightarrow * A^{*}$ is a rank-1 specialization. Since $\left(a_{i}^{*} b_{j}^{*} e_{i j}^{*} a_{0} b_{0}\right) \rightarrow\left(a_{0} b_{0} e_{i 1} a_{0} b_{0}\right), \operatorname{rk}\left(a_{1}^{*} e_{1 j}^{*}\right) \geq \operatorname{rk}\left(a_{0} e_{i 1}\right)=3$, so equality holds; similarly $\operatorname{rk}\left(a_{1}^{*} b_{j}^{*} e_{i j}^{*}\right)=3$, so $b_{j}^{*} \in \operatorname{acl}\left(a_{1}^{*} e_{i j}^{*}\right)$. Hence $\operatorname{rk}\left(\left(a_{1}^{*} b_{j}^{*} e_{i j}^{*} a_{0} b_{0}\right)=\right.$ $\operatorname{rk}\left(a_{1}^{*} e_{i j}^{*} a_{0} b_{0}\right)=\operatorname{rk}\left(a_{0} e_{i j}^{*} a_{0} b_{0}\right)=3$. So $\left(a_{1}^{*} b_{j}^{*} e_{i j}^{*} a_{0} b_{0}\right) \rightarrow\left(a_{0} b_{0} e_{i 1} a_{0} b_{0}\right)$ is an isomorphism. Hence $b_{j}^{*}=b_{0}$. Dually one shows now that each $a_{i}^{*}=a_{0}$. Thus $A_{i j}^{*}=\left(a_{0} b_{0} e_{i j}^{*}\right)$. Now $A^{*} \rightarrow * A^{\prime \prime}$ also gives $e_{i j}^{*} e_{i^{\prime} j^{\prime}}^{*} \rightarrow * e_{i 1} e_{i^{\prime} 1}$ so if $i \neq i^{\prime}$ (since $\operatorname{rk}\left(e_{i 1} e_{i^{\prime} 1} / a_{0} b_{0}\right)=2$ and $\operatorname{rk}\left(e_{i j}^{*} e_{i^{\prime} j^{\prime}}^{*} / a_{0} b_{0}\right) \leq 2$ ) we have $\operatorname{tp}\left(e_{i j}^{*} e_{i^{\prime} j^{\prime}}^{*} / a_{0} b_{0}\right)=$ $\operatorname{tp}\left(e_{i 1} e_{i^{\prime} 1} / a_{0} b_{0}\right)$. Thus $\operatorname{stp}\left(e_{i j}^{*} / a_{0} b_{0}\right)=\operatorname{stp}\left(e_{i^{\prime} j^{\prime}}^{*} / a_{0} b_{0}\right)=q$ (say), and of course this remains true if $i=i^{\prime}$. It follows that $\operatorname{stp}\left(A_{i j}^{*} / a_{0} b_{0}\right)$ does not depend on $i, j$, and has rank 1.

(iii) follows from 6.8 once we verify the "goodness" hypothesis there. We must show that $\left(a_{0}, b_{0}, a_{i}, a_{i}^{\prime}, b_{j}, e_{i j}, e_{i^{\prime} j}\right) \rightarrow\left(a_{0}, b_{0}, a_{0}, a_{0}, b_{0}, e_{i j}^{*}, e_{i^{\prime} j}^{*}\right)$ is good. Now $\left(a_{0}, b_{0}, b_{j}, e_{i j}, e_{i^{\prime} j}\right) \rightarrow\left(a_{0}, b_{0}, b_{0}, e_{i j}^{*}, e_{i^{\prime} j}^{*}\right)$ is a rank-1 specialization, and is regular by 6.6(7). (Indeed $\left(a_{0}, b_{0}, e_{i j}, e_{i^{\prime} j}\right) \rightarrow\left(a_{0}, b_{0}, e_{i j}^{*}, e_{i^{\prime} j}^{*}\right)$ is an isomorphism, $b_{j} \rightarrow b_{0}$ is a regular rank-1 specialization, and $b_{j}$ is independent from $\left(a_{0}, b_{0}, e_{i j}, e_{i^{\prime} j}\right)$.) Also $\left(b_{j}, e_{i j}\right) \rightarrow\left(b_{0}, e_{i j}^{*}\right)$ is an isomorphism, and $a_{i} \in \operatorname{acl}\left(b_{j}, e_{i j}\right)$; similarly $\left(b_{j}, e_{i^{\prime} j}\right) \rightarrow$ $\left(b_{0}, e_{i^{\prime} j}^{*}\right)$ is an isomorphism, and $a_{i^{\prime}} \in \operatorname{acl}\left(b_{j}, e_{i^{\prime} j}\right)$. Hence the specialization is good by the recursive definition of goodness.

Claim 4. With $M$ still fixed, in Claim 3 there is only a finite number of possibilities for $\operatorname{tp}\left(A^{*} / a_{0} b_{0}\right)$.

Proof. The locus of $A^{*}$ over $a_{0} b_{0}$ is a component of the intersection of the locus of $A$ over $a_{0} b_{0}$, with the diagonal corresponding to " $a_{1}^{*}=a_{0}^{*}$ ". There are only finitely many such components.

Now let $P_{m}$ be the disjunction of the possible types in Claim 4. By compactness, there exists an infinite array $A^{*}=\left(A_{i j}^{*}: i, j=1,2, \ldots\right)$ such that $P_{m}$ holds for every $m \times m$ square from $A^{*}$. By 6.6(1) we may assume $A^{*}$ is indiscernible. By 6.3(a), there exists a definable one-dimensional group. 
In the following lemma, the reader may read "ample Zariski geometry" for "not locally modular"; or see [Hr2].

Lemma 6.11. If $D$ is not locally modular, then $D$ interprets a one-dimensional field.

Proof. By 6.10 D interprets a one-dimensional Abelian group $H$. By $5.11 H$ may be given a manifold structure, so that addition is a morphism. Let $C(e) \subseteq(H \times H)(e \in$ $E)$ be a two-dimensional family. This may be obtained from a two-dimensional family of subsets of $D \times D$ using a finite-to-finite correspondence between $D$ and $H$. Let $C^{2}$ have the same meaning as in 6.10 , so that (i), (ii) hold.

Let $a_{i}, b_{i}, b^{j}(i, j=0,1,2, \ldots)$ be independent generic elements of $H, b_{i j}=b_{i}+b^{j}$. Let $I=J=\{1,2, \ldots\}$; from now on the indices $i, j$ will range over $I, J$. For $i, j \geq 1$ there exists $e_{i j} \in E$ such that $C^{2}\left(e_{i j} ; a_{i} b_{i j}, a_{0} b_{00}\right)$. Let $A_{i j}=\left(a_{i}, b_{i j}, e_{i j}\right), A=$ $\left(A_{i j}\right)_{i j \geq 1}$. We may choose $e_{i j}$ so that $A$ is indiscernible. We have $e_{i j} \in \operatorname{acl}\left(a_{i}, b_{i j}\right)$, so any $m \times n$ rectangle has rank $2 m+n-1$; this remains true over $\left\{a_{0} b_{00}\right\}$. Write $x \rightarrow * y$ for $\left(x, a_{0}, b_{00}\right) \rightarrow\left(y, a_{0}, b_{00}\right)$.

Claim 1. Let $A_{i j}^{\prime}=A_{1 j}$. Then $A \rightarrow * A^{\prime}$.

Proof. $\left\{\left(a_{i}, b_{i}\right): i \in I\right\}$ is independent over $B=\left\{b^{j}: j \in J\right\} \cup\left\{a_{0}, b_{0}\right\}$. Clearly $\left(e_{i j}, b_{i j}\right) \in \operatorname{acl}\left(B \cup\left\{a_{i}, b_{i}\right\}\right)$. Hence $\left(a_{i}, b_{i J}, e_{i J}\right)_{i \in I}$ is independent over $B$. Indiscernibility is also clear, so 6.6(4) applies.

Claim 2. Let $A_{i j}^{\prime \prime}=\left(a_{0}, b_{00}, e_{1 j}\right)$. Then $A^{\prime} \rightarrow * A^{\prime \prime}$.

Proof. It suffices to show that $\left(\left(a_{1}, b_{1 j}, e_{1 j}\right): j\right) \rightarrow *\left(\left(a_{0}, b_{00}, e_{1 j}\right): j\right)$. For each $j$ individually this follows from property (ii) of $C^{2}$ (in Lemma 6.10). Note that $\left(\left(a_{1}, b_{1 j}, e_{1 j}\right): j\right)$ is indiscernible and independent over $\left(a_{0}, b_{00}, a_{1}\right)$. Since $\left(a_{0}, b_{00}, a_{1}\right) \rightarrow\left(a_{0}, b_{00}, a_{0}\right)$ by a good rank-1 specialization, 6.6(4) applies.

Now fix an integer $M$, and restrict attention to indices $\leq M$.

Claim 3. There exists $A^{*}=\left(A_{i j}^{*}\right)_{i, j}$ and a rank-1 specialization $A \rightarrow * A^{*}$, such that:

(i) $\operatorname{stp}\left(A_{i j}^{*} / a_{0} b_{00}\right)$ does not depend on $i, j$, and has rank 1 . Each row $A_{i J}^{*}$ is independent over $\left\{a_{0} b_{00}\right\}$.

(ii) $A_{i j}^{*}=\left(a_{0} b_{00} e_{i j}^{*}\right)$ for some $e_{i j}^{*}$.

(iii) $A^{*}$ is rank indiscernible, of type $2 m+n-2$.

Proof. By 6.6(3) there exists $A^{*}=\left(A_{i j}^{*}\right), A_{i j}^{*}=\left(a_{i}^{*}, b_{i j}^{*}, e_{i j}^{*}\right)$, such that $A \rightarrow$ $* A^{*} \rightarrow * A^{\prime \prime}, a_{1}^{*}=a_{0}$ and $A \rightarrow * A^{*}$ is a rank-1 specialization. We will show (i)-(iii) hold.

$\left(\mathrm{i}^{\prime}\right)$ We first show a weak version of $(\mathrm{i}): \operatorname{rk}\left(e_{i j}^{*} / a_{0} b_{00}\right)=1$, and each row $\left(e_{i J}^{*}\right)$ is independent over $\left\{a_{0} b_{00}\right\}$. Now $e_{i J} \rightarrow * e_{i J}^{*} \rightarrow * e_{1 J} ; \operatorname{since} \operatorname{rk}\left(e_{i J} / a_{0} b_{00}\right)=$ $\operatorname{rk}\left(e_{1 J} / a_{0} b_{00}\right)$ these specializations are isomorphisms, and the statement follows.

(ii') Next, a special case of (ii): $b_{1 j}=b_{00}$ for each $j$. Note first that $\left(a_{i}^{*}, b_{i j}^{*}, e_{i j}^{*}\right) \rightarrow$ $\left(a_{0}, b_{00}, e_{i j}\right)$; since $\left(a_{0}, b_{00}, e_{i j}\right)$ is a generic point of $C$, so is $\left(a_{i}^{*}, b_{i j}^{*}, e_{i j}^{*}\right)$. In particular $b_{i j}^{*} \in \operatorname{acl}\left(a_{i}^{*}, e_{i j}^{*}\right)$. Thus

$$
\operatorname{rk}\left(a_{1}^{*} b_{1 j}^{*} e_{1 j}^{*} / a_{0} b_{00}\right)=\operatorname{rk}\left(a_{1}^{*} e_{1 j}^{*} / a_{0} b_{00}\right)=\operatorname{rk}\left(a_{0} e_{1 j}^{*} / a_{0} b_{00}\right) \leq 1
$$

Now $e_{1 j}^{*} \rightarrow * e_{1 j}$, and $\operatorname{rk}\left(a_{0} b_{00} e_{1 j} / a_{0} b_{00}\right)=1$. Thus the specialization $a_{1}^{*} b_{1 j}^{*} e_{1 j}^{*} \rightarrow$ $* a_{0} b_{00} e_{1 j}$ is an isomorphism. So $b_{1 j}^{*}=b_{00}$.

(ii) By definition of $b_{i j}$, we have $b_{1 j}-b_{1 j^{\prime}}=b_{i j}-b_{i j^{\prime}}$; by $6.6(6), 0=b_{00}-b_{00}=$ $b_{1 j}^{*}-b_{1 j^{\prime}}^{*}=b_{i j}^{*}-b_{i j^{\prime}}^{*} ;$ so $b_{i j}^{*}=b_{i j^{\prime}}^{*}$ for any $i, j, j^{\prime}$. 
Let $i \in I$ and let $j, j^{\prime}$ be distinct. Then $\left(a_{i}^{*}, b_{i j}^{*}\right)=\left(a_{i}^{*}, b_{i j^{\prime}}^{*}\right)$ is a common point of $C\left(e_{i j}^{*}\right)$ and $C\left(e_{i j^{\prime}}^{*}\right)$. By $\left(\mathrm{i}^{\prime}\right), e_{i j}^{*}$ and $e_{i j^{\prime}}^{*}$ are distinct, so $C\left(e_{i j}^{*}\right)$ and $C\left(e_{i j^{\prime}}^{*}\right)$ are distinct curves, and so $\left(a_{i}^{*}, b_{i j}^{*}\right) \in \operatorname{acl}\left(e_{i j}^{*}, e_{i j^{\prime}}^{*}\right)$. Thus

$$
\operatorname{rk}\left(a_{i}^{*} b_{i j}^{*} b_{i j^{\prime}}^{*} e_{i j}^{*} e_{i j^{\prime}}^{*} / a_{0} b_{00}\right) \leq 2 .
$$

Now $a_{i}^{*} b_{i j}^{*} b_{i j^{\prime}}^{*} e_{i j}^{*} e_{i j^{\prime}}^{*} \rightarrow * a_{0} b_{00} b_{00} e_{1 j} e_{1 j^{\prime}}$. Since $\operatorname{rk}\left(e_{1 j} e_{1 j^{\prime}} / a_{0} b_{00}\right)=2$, this specialization is also an isomorphism; so $b_{i j}^{*}=b_{00}$ and $a_{i}^{*}=a_{0}$.

To verify the "goodness" hypothesis in 6.9 , over $a_{0} b_{00}$, recall that

$$
a_{0} b_{00} A_{i j} A_{i j^{\prime}} A_{i j^{\prime \prime}}=\left(a_{0} b_{00} a_{i} b_{i j} b_{i j^{\prime}} b_{i j^{\prime \prime}} e_{i j} e_{i j^{\prime}} e_{i j^{\prime \prime}}\right) \text {. }
$$

We have $b_{i j} \in \operatorname{acl}\left(a_{i} e_{i j}\right)$ etc., and $a_{i} e_{i j} \rightarrow a_{0} e_{i j}^{*}$ is an isomorphism. Further $\left(a_{0} b_{00} e_{i j} e_{i j^{\prime}} e_{i j^{\prime \prime}}\right) \rightarrow\left(a_{0} b_{00} e_{i j}^{*} e_{i j^{\prime}}^{*} e_{i j^{\prime \prime}}^{*}\right)$ and $a_{i} \rightarrow a_{0}$ are regular (being isomorphisms), and $a_{i}$ is free from $\left(a_{0} b_{00} e_{i j} e_{i j^{\prime}} e_{i j^{\prime \prime}}\right)$, so

$$
\left.a_{0} b_{00} a_{i} e_{i j} e_{i j^{\prime}} e_{i j^{\prime \prime}}\right) \rightarrow\left(a_{0} b_{00} a_{0} e_{i j}^{*} e_{i j^{\prime}}^{*} e_{i j^{\prime \prime}}^{*}\right)
$$

is regular, by $6.6(7)$.

Now (i) follows from (i') and (ii). (iii) follows from 6.9.

The rest of the proof, from Claim 4 on, is identical to the proof of 6.10 (using $6.3(\mathrm{~b})$ instead of $6.3(\mathrm{a}))$.

\section{Purity of the Algebraic structure}

In $\S 6$ we found a one-dimensional manifold $F$ carrying a field structure. Since addition and multiplication are morphisms, it is clear that any polynomial on $F^{n}$ is a morphism, and hence any subset of $F^{n}$ defined by the vanishing of polynomials is closed. We must now show that there are no other closed sets. Until we show that the two topologies coincide, we will refer to the usual Zariski topology as $T_{a}$ and use the adjective "algebraic" to refer to it. The topology induced on the manifold from the Zariski geometry structure will be denoted $T_{b}$.

7.1. Completeness of projective space. In the algebraic category projective space is complete: for any manifold $X$, if $C \subseteq X \times \mathbf{P}^{n}$ is closed, then it projects to a closed set. We do not immediately show this in the category $T_{b}$, but we show that $\mathbf{P}^{n}$ is weakly complete: for any manifold $X$, if $C \subseteq X \times \mathbf{P}^{n}$ is closed and projects to a dense subset of $X$, then it projects onto $X$. Post facto, we will know that every closed subset of $\Pi$ is algebraic, and so $\mathbf{P}^{n}$ is complete at least with respect to algebraic $X$; we have not checked the general case.

We recall the construction of projective space $\mathbf{P}^{d}$ over a field $F$ (see e.g. [Ha]). Consider $V=F^{d+1}$ as a $(d+1)$-dimensional vector space over $F$. Let $V^{\prime}=V-(0)$. Let $\mathbf{P}^{d}$ be the set of lines through 0 in $V$. Define a map $\theta: V^{\prime} \rightarrow \mathbf{P}^{d}$ by sending a point $v$ to the unique line $\theta v$ through 0 and $v$. We denote $\theta\left(x_{0}, \ldots, x_{d}\right)$ by $\left(x_{0}: \cdots: x_{d}\right)$.

We make $\mathbf{P}^{d}$ into a manifold as follows. Let $E_{i}=\left\{\left(x_{0}, \ldots, x_{d}\right) \in V: x_{i}=0\right\}$, a subspace of codimension 1 . Let $X_{i}$ be the set of lines contained in $E_{i}$. Then $\bigcap_{\iota} E_{i}=\emptyset$. Cover $\mathbf{P}^{d}$ by the sets $U_{i}=\mathbf{P}^{d}-X_{i}$; and let $\alpha_{i}: U_{i} \rightarrow F^{d}$ be the map sending the line through $\left(x_{1}, \ldots, x_{i-1}, 1, x_{i}, \ldots, x_{d}\right)$ to $\left(x_{1}, \ldots, x_{d}\right)$. The transition maps $F^{d} \rightarrow F^{d}$ are morphisms since inversion on $F-(0)$ and multiplication of $F \times F$ are morphisms. Since $F^{d}$ is a manifold, this gives a manifold structure to $\mathbf{P}^{d}$ also. Moreover $\theta$ is a morphism, and in particular $\mathbf{P}^{d}$ is $T_{b}$-irreducible. 
Proposition 7.1. Let $X$ be a manifold, $C \subseteq X \times \mathbf{P}^{n}$ be closed, and assume $\pi_{X} C$ is dense in $X$. Then $C$ projects onto $X$.

Proof. Let $Y=\mathbf{P}^{n}$. Replacing $C$ by an irreducible component projecting onto a dense subset of $X$, if necessary, we may assume $C$ is irreducible. Let $V, V^{\prime}, \theta$ : $V^{\prime} \rightarrow Y$ be vector spaces and maps as above, corresponding to the projective space structure of $Y$. Let $\theta$ also denote the map from $X \times V^{\prime}$ to $X \times Y$ given by $\theta(x, v)=(x, \theta v)$. Let $C^{*}$ be the closure in $X \times V$ of $\theta^{-1} C$. Since $\theta$ is a morphism, $\theta^{-1} C$ is closed in $X \times V$, so $C^{*} \cap\left(X \times V^{\prime}\right)=\theta^{-1} C$.

For $\alpha \in F, v \in V$ and $x \in X$, write $\alpha \cdot(x, v)$ for $(x, \alpha v)$. This is a morphism on $X \times V$; if $\alpha \in F^{x}$, it is an isomorphism.

Claim. If $(x, v) \in C^{*}$ and $\alpha \in F^{x}$, then $(x, \alpha v) \in C^{*}$.

Proof. If $(a, v) \in\left(\theta^{-1} C\right)$, then for any $\alpha \in F^{x},(a, \alpha v) \in\left(\theta^{-1} C\right)$; thus $\left(\theta^{-1} C\right) \subseteq$ $\alpha^{-1}\left(\theta^{-1} C\right) \subseteq \alpha^{-1} C^{*}=\left\{(x, v):(x, \alpha v) \in C^{*}\right\}$; since this last set is closed and contains $\theta^{-1} C$, it must contain $C^{*}$.

We have $\operatorname{dim} C^{*}=\operatorname{dim}(C)+1$. Let $Z$ be a component of $C^{*}$ of maximal dimension. Then $\alpha^{-1} Z$ is also a component of $C^{*}$ of maximal dimension. Thus $F^{x}$ acts on the set $\left\{Z_{1}, \ldots, Z_{m}\right\}$ of such components. Hence $\left\{\alpha \in F^{x}: \alpha^{-1} Z_{i}=Z_{i}\right.$ for each $i\}$ has index at most $m$ ! in $F^{x}$. It is a closed subgroup, hence equal to $F^{x}$. Fix a component $Z$ of $C^{*}$ of maximal dimension. Then $\operatorname{dim} Z>\operatorname{dim}(X \times(0))$, so $\operatorname{dim}\left(Z \cap\left(X \times V^{\prime}\right)\right)=\operatorname{dim}(C)+1$. Thus $\theta\left(Z \cap\left(X \times V^{\prime}\right)\right)$ is a dense subset of $C$; so it projects to a dense subset of $X$. Let $a \in X$ be a generic element. Then there exists $b \in Z(a)$. Since $Z$ is $F^{x}$-invariant, $F^{x} b \subseteq Z(a)$. But $Z(a)$ is closed, so $F b \subseteq Z(a)$. Thus $(b, 0) \in Z$. So $\{x \in X:(x, 0) \in Z\}$ contains a generic element of $X$, hence equals $X$. We have shown that for any $a \in X, Z(a) \neq \emptyset$. But by the dimension theorem in $X \times V, \operatorname{dim} Z(a) \geq \operatorname{dim} Z-\operatorname{dim} X=1$. Hence $Z(a)$ is infinite; so there exists $(a, v) \in Z, v \neq 0$. Thus $(a, v) \in C^{*} \cap\left(X \times V^{\prime}\right)=\theta^{-1} C$. So $(a, \theta v) \in C$, showing that $a \in \pi_{X} C$. So $C$ projects onto $X$, as required.

We now make the assumption that $F=D$ (as sets). This assumption has the effect of limiting the number of manifolds $Y$ considered in the definition of completeness; the assumption is harmless for the purposes of this section. We did not investigate the situation without it, except for 7.4 below which will be needed later.

By a $T_{a}$-manifold we will mean one locally modelled on $T_{a}$-regular subsets of $F^{n}$. Then it is clear that any $T_{a}$-manifold can also be viewed as a $T_{b}$-manifold, and $T_{a}$-morphisms are $T_{b}$-morphisms. We require the following improvement.

Lemma 7.2. Assume $F=D$. Let $M, N$ be $T_{a}$-manifolds. Assume $M$ is isomorphic as an algebraic variety to a subvariety $M^{\prime}$ of $N$, via a map $g: M \rightarrow N$. Then $g: M \rightarrow M^{\prime}$ is a closed map in $T_{b}$. Moreover if $Y$ is a $T_{b}$-manifold then the map $\left(\operatorname{id}_{Y}, g\right): Y \times M \rightarrow Y \times M^{\prime}$ is a closed map.

Proof. We have an open covering of $M$ by open affine subsets $M_{i}$, and of $N$ by open affine subsets $N_{j}$. We may choose the sets $N_{j}$ small enough that $g^{-1} N_{j}$ is contained in some $M_{i}$. It suffices to show that $g$ is closed on each $g^{-1} N_{j}$; hence we may assume $M, N$ are affine. Thus we may view $M, N$ as $T_{a}$-regular subsets of $F^{m}, F^{n}$ respectively. The fact that $g$ is an isomorphism implies that for each coordinate map $x_{i}$ on $F^{m}$, the map $x_{i} g^{-1}$ is a regular map on $M^{\prime}$, and hence there exists a rational map $r_{i}$ on $F^{n}$ with no poles on $M^{\prime}$ such that $r_{i}=x_{i} g^{-1}$ on $M^{\prime}$. The map $r=\left(r_{1}, \ldots, r_{m}\right)$ is a morphism from its domain to $F^{m}$, since the field 
operations are morphisms; and $r$ coincides with $g^{-1}$ on $M^{\prime}$. Now let $X \subseteq M$ be closed. Let $X^{\prime}=\operatorname{cl}(X)$ taken in $F^{m}$. Then $X=M \cap X^{\prime}$, and $f X=M^{\prime} \cap r^{-1} X^{\prime}$. Since $r$ is a morphism, $X^{\prime}$ is closed in $\operatorname{dom}(r)$, so $g X$ is closed in $M^{\prime}$. The moreover clause follows similarly, using 5.9 .

From this we can deduce:

Proposition 7.3. Assume $F=D$. Let $M$ be a $T_{a}$-manifold, algebraically isomorphic to a projective variety. Then $M$ is weakly complete.

Proof. Let $f: M \rightarrow M^{\prime}$ be an isomorphism, $M^{\prime}$ a subvariety of $\mathbf{P}^{d}$. Let $Y$ be a $T_{b}$-manifold. Let $C \subseteq Y \times M$ be closed, and assume $C$ projects onto a dense subset of $Y$. Let $F(y, m)=(y, f(m))$. Then by $7.2, F$ is a closed map into $Y \times M^{\prime}$, so $F(C)$ is closed in $Y \times M^{\prime}$. Since $M^{\prime}$ is closed in $\mathbf{P}^{d}, F(C)$ is closed in $Y \times \mathbf{P}^{d}$. The projection of $F(C)$ to $Y$ coincides with the projection of $C$ to $Y$, so is dense in $Y$, and hence by 7.1 equals $Y$.

Lemma 7.4. Let $Y$ be a projective algebraic variety and $X$ a $T_{b}$-manifold, both irreducible of dimension one. Let $h$ be a closed irreducible subset of $X \times Y$, and assume $h$ is the graph of a partial function from $X$ to $Y$, with $\operatorname{dom}(h)$ dense in $X$. Then $h$ is total, $\operatorname{dom}(h)=X$.

Proof. $Y$ can be embedded in $\left(\mathbf{P}^{1}\right)^{n}$ for some $n$, and we will view it that way. Let $\pi_{i}$ be the projections from $\left(\mathbf{P}^{1}\right)^{n}$ to $\mathbf{P}^{1}, f_{i}=\pi_{i} h$, and $F_{i}$ the Zariski closure of the graph of $f_{i}$. Note that $f_{i}$ is the projection to $X \times \mathbf{P}^{1}$ of the intersection of copies of $\pi_{i} \times Y$ and of $h \times \mathbf{P}^{1}$ on $X \times \mathbf{P}^{1} \times\left(\mathbf{P}^{1}\right)^{n}$, so every component of this intersection has dimension at least one, hence it must be irreducible of dimension one, and it follows that $F_{i}$ is irreducible. By $7.1 F_{i}$ projects onto $X$. Since the dimension is one there is no exceptional locus, and by $4.8 F_{i}$ is the graph of a function $X \rightarrow \mathbf{P}^{1}$. We can put the $F_{i}$ back together into a morphism $F: X \rightarrow\left(\mathbf{P}^{1}\right)^{n}$. This morphism agrees with $h$ generically, and hence must be equal to $h$.

7.2. Irreducibility. A good way to satisfy the dimension theorem would appear to be to let a $T_{b}$-closed set be a certain kind of open subset of a $T_{a}$-closed set. Every $T_{a}$-closed set would be $T_{b}$-closed, but in general reducible. We must rule out this scenario. On the constructible level it was shown in [Hr4], Theorem 1, that the notion of irreducibility does not change, but here we must take smaller-dimensional components into account, and so the argument must be somewhat refined.

Proposition 7.5. Let $X$ be a closed, irreducible subset of $F^{n}$ in the $T_{a}$-topology. Then it is closed, irreducible in the $T_{b}$-topology.

Here we may restrict attention to subsets of $F^{n}$, so we may assume that $D=F$ (as sets). Hence any $T_{a}$-manifold is a $T_{b}$-manifold.

Lemma 7.6. If an algebraic curve $C \subseteq \mathbf{P}^{n}$ is $T_{a}$-irreducible, then it is $T_{b^{-}}$ irreducible.

Proof. We use the following algebraic facts; all refer to $T_{a}$. The reader is referred to any book on algebraic geometry, e.g. Lang's Introduction to algebraic geometry $[\mathrm{L}]$.

(i) There exists a smooth curve $C^{\prime} \subseteq \mathbf{P}^{3}$ and a morphism from $C^{\prime}$ onto $C$.

(ii) If $C$ is a smooth curve, there exists an integer $g$ associated with $C$, the genus of $C$, with the following properties. 
(a) If $g=0$, then there exists a $T_{a}$-morphism $\mathbf{P}^{1} \rightarrow \mathbf{P}^{3}$ whose image is $C$.

(b) If $g>0$, then there exists a variety $J$ of dimension $g$ admitting an algebraic group structure, with zero point $0 . \quad J$ is a divisible Abelian group. Moreover,

(c) There exists a surjective morphism $h: C^{g} \rightarrow J$ such that:

$(*)$ for all $c_{1} \in C$, for all $\left(c_{2}, \ldots, c_{g}\right)$ in some dense open subset $H$ of $C^{g-1}$, for all $d_{1}, \ldots, d_{g} \in C^{g}$, if $h\left(c_{1}, \ldots, c_{g}\right)=h\left(d_{1}, \ldots, d_{g}\right)$, then $d_{i}=c_{\sigma(i)}$ for some permutation $\sigma$ of $\{1, \ldots, g\}$.

Remark 7.7. By 5.11 and 5.12 , we may view $J$ as a $T_{a}$-manifold, in a unique way; this also makes it into a $T_{b}$-manifold. Since $J$ is divisible, it has no proper subgroups of finite index, and hence by $5.12(\mathrm{a}) J$ is irreducible as a $T_{b}$-manifold. We will use this to prove the lemma.

Remark 7.8 (and proof of (c)). (c) states that outside of some proper closed subset $F$ of $C^{g}, h$ identifies two $g$-tuples iff one is a permutation of the other; moreover, no translate of the divisor $C+\cdots+C$ (added $g-1$ times in $J$ ) is contained in $h F$. For lack of a reference to the latter statement, we provide a proof.

We use the following further facts, see $[\mathrm{L}]$. A divisor is a formal sum of elements of $C$, with integer coefficients. If $f: C \rightarrow \mathbf{P}^{1}$ is a rational function, then $Z(f)$ is the formal sum of its zeroes, counted with multiplicities, $P(f)=Z(1 / f),(f)=$ $Z(f)-P(v)$ (a divisor).

(d) For $2 g$ elements of $C$, we have $h\left(x_{1}, \ldots, x_{g}\right)=h\left(y_{1}, \ldots, y_{g}\right)$ iff there exists a rational function $f: C \rightarrow \mathbf{P}^{1}$ such that

$$
(f)=x_{1}+\cdots+x_{g}-y_{1}-\cdots-y_{g}
$$

(as formal divisors.)

(e) (Weak Riemann-Roch.) Given a set $s$ of $t$ points of $C$, let $L(s)$ be the space of rational functions $f: C \rightarrow \mathbf{P}^{1}$ whose poles have multiplicity 1 , and are among the points of $s$. Then $L(s)$ is a linear space, of $F$-dimension $t-g+1$ if $t \geq 2 g-1$.

To prove (c), let $E=\left\{x \in C^{g}\right.$ : for some $y$, not a permutation of $\left.x, h(y)=h(x)\right\}$. We must show that $\operatorname{cl}(E)$ is a proper subset of $C^{g}$, and moreover:

$$
\left(c, x_{2}, \ldots, x_{g}\right) \notin E \quad \text { for generic } x_{2}, \ldots, x_{g} .
$$

Suppose for contradiction that $\left(c, x_{2}, \ldots, x_{g}\right) \in E$ for generic $x_{2}, \ldots, x_{g}$. Then $h\left(c, x_{2}, \ldots, x_{g}\right)=h\left(y_{1}, \ldots, y_{g}\right)$, where $\left(c, x_{2}, \ldots, x_{q}\right)$ is not a permutation of $\left(y_{1}, \ldots, y_{q}\right)$. By $(\mathrm{d})$, there exists a nonconstant rational function $f: C \rightarrow \mathbf{P}^{1}$ with poles at most at $\left\{c, x_{2}, \ldots, x_{g}\right\}$, and there poles of multiplicity 1 . Thus $L\left(c, x_{2}, \ldots, x_{q}\right)$ contains nonconstant functions. If $L(c)$ contains a nonconstant function $f$, then $f$ takes the value $\infty$ at only one point, hence is a bijection between $C$ and $\mathbf{P}^{1}$, so $g=0$, contrary to assumption. Thus there exists $i<g$ such that $L\left(c, x_{2}, \ldots, x_{i}\right)$ contains only constant functions, while $L\left(c, x_{2}, \ldots, x_{i}, x_{i+1}\right)$ contains a nonconstant one. Let $L^{*}(U)$ denote $L\left(c, x_{2}, \ldots, x_{i}, U\right)$. Then $L^{*}(\emptyset)$ is one-dimensional, while $L^{*}(y)$ is (at least) two-dimensional for generic $y \in C$. Let $Y$ be a set of $2 g$ mutually generic elements of $C$. Evidently for any $y \in Y$, $L^{*}(y) \cap L^{*}(Y-\{y\})=L^{*}(\emptyset)$; so we have a direct sum of $2 g$ subspaces $L^{*}(y)$ over the constants in $L^{*}(Y)$, and hence this space has dimension $\geq 2 g+1>(2 g+i-g+1)$. This contradicts (e). 
We proceed to prove the lemma. By (i) it suffices to prove that $C^{\prime}$ is irreducible; so we may assume $C$ is smooth. We know that the strongly minimal set $\mathbf{P}^{1}$ is irreducible, since it was obtained by glueing together two irreducible open sets. (One could also argue using the descending chain condition on closed sets.) Thus any continuous image of $\mathbf{P}^{1}$ is irreducible. Hence by (ii)(a) we may assume the genus of $C$ is at least 1 . Let $J$ be as in (ii)(b,c), and let $F$ be a connected component of the graph of $h$ projecting to a dense subset of $J$. Consider $F$ as a subset of $J \times \mathbf{P}^{3} \times$ $\cdots \times \mathbf{P}^{3}$. The hypotheses of refprop:seventhree are verified; so $F$ projects onto $J$. Now suppose $C$ has an infinite, proper, $T_{b}$-closed subset $Y$. Then we may choose $F \subseteq(J \times Y \times \cdots \times Y)$. But then any point in $\pi_{J} F$ has the form $h\left(y_{1}, \ldots, y_{g}\right)$ where each $y_{i}$ is in $Y$. Picking $c \notin Y$, and generic $x_{2}, \ldots, x_{q}$, we have $h\left(c, x_{2}, \ldots, x_{q}\right)=$ $h\left(y_{1}, \ldots, y_{g}\right)$ with $y_{i} \in Y$, and in particular $y_{i} \neq c$. Thus $\left(c, x_{2}, \ldots, x_{q}\right)$ is not a permutation of $\left(y_{1}, \ldots, y_{g}\right)$, contradicting (c). This contradiction shows that $C$ is irreducible.

Lemma 7.9. If an algebraic set $C \subseteq \mathbf{P}^{n}$ is $T_{a}$-irreducible, then it is $T_{b}$-irreducible.

Proof. As was noted above, by Theorem 1 of [Hr4], $C$ has at most one $T_{b}$-component $C^{\prime}$ of maximal dimension. We must show that $C=C^{\prime}$. If $\operatorname{dim}(C)=1$ we are done; otherwise we use induction on the dimension. Let $a \in C$. Let $C^{\prime \prime}=\operatorname{cl}\left(C^{\prime}-C\right)$. We have $\operatorname{dim}\left(C^{\prime \prime}\right)<\operatorname{dim}(C)$. Choose a generic linear hypersurface $H$ through $a$; then $\operatorname{dim}\left(C^{\prime \prime} \cap H\right) \leq \max \left(0, \operatorname{dim}\left(C^{\prime \prime}\right)-1\right)$. Let $E$ be a $T_{a}$-component of $C \cap H$ passing through $a$. Then $\operatorname{dim}(E) \geq \operatorname{dim}(C)-1 \geq \max \left(1, \operatorname{dim}\left(C^{\prime \prime}\right)\right)$. So $E$ is not contained in $C^{\prime \prime}$. By induction, $E$ is $T_{b}$-irreducible. Since $E \subseteq C=C^{\prime \prime} \cup C^{\prime}, E$ is contained in $C^{\prime}$. This shows that $a \in C^{\prime}$. Since $a \in C$ was arbitrary, $C^{\prime}=C$.

\subsection{The purity argument.}

Proposition 7.10. Any closed subset of $F^{n}$ is algebraic.

We first concern ourselves with curves in $\mathbf{P}^{2}$. Consider the algebraic curves. Such a curve is defined by an equation:

$$
C(a)=\left\{(x: y: z) \in \mathbf{P}^{2}: \sum a_{i j} x^{i} y^{j} z^{d-i-j}=0\right\},
$$

where $a=\left(a_{i j}: i+j \leq d\right)$ is in $F^{d(d+1) / 2}$. Note that $C(a)$ is actually determined by the projective point $\theta a$. Thus if $q(d)=(d+1)(d+2) / 2-1$, we have a closed set $C=C_{d} \subseteq \mathbf{P}^{q(d)} \times \mathbf{P}^{2}$,

$$
C=\left\{\left(\theta\left(a_{i j}: i+j \leq d\right),(x: y: z)\right): \sum a_{i j} x^{i} y^{j} z^{d-i-j}=0\right\}
$$

such that $C(a)$ runs through all algebraic curves of degree $d$ in $\mathbf{P}^{2}$.

When we think of $\mathbf{P}^{q(d)}$ as coordinatizing curves of degree $d$, we will write $\mathbf{Q}^{d}=$ $\mathbf{P}^{q(d)}$. If $d=1$, then the curves $C(a)$ are called lines. When $d \geq 2$, the curves $C(a)$ are not necessarily reducible. In particular, any union of $d$ lines has the form $C(a)$ for some $a$. The set of points $a \in \mathbf{Q}^{d}$ such that $C(a)$ is the union of lines is a $T_{a}$-closed subset, of dimension $2 d$. We denote it by $\mathbf{L}^{d}$. If $a \in \mathbf{L}^{d}, C_{d}(a)$ is the union of $d$ lines, not necessarily all distinct; a point of $C_{d}(a)$ lying on more than one of these is called a double point of $C_{d}(a)$.

We will use the fact that the dimension theorem holds in $\mathbf{P}^{n}$ (it is locally $F^{n}$ ). We will also use the following purely algebraic facts. Both could be shown either explicitly (partial derivatives) or using dimension-theoretic arguments. The second 
fact states essentially that a line is tangent to a curve in $\mathbf{L}^{d}$ iff it is one of the component lines, or passes through a double point.

Lemma 7.11. For any $q(d)$ points in $\mathbf{P}^{2}$ there exists $a \in \mathbf{Q}^{d}$ such that $C_{d}(a)$ passes through all $q(d)$ points.

Proof. Let points $\left(x_{s}: y_{s}: z_{s}\right)(s=1, \ldots, q(d))$ be given. Let $R=\{(i, j, k)$ : $i+j+k=d\}$. For $r=(i, j, k) \in R$, let $c_{r s}=x_{s}^{i} y_{s}^{j} z_{s}^{k}$. Then $\left(c_{r s}\right)$ is a matrix of size $q(d)-1$ by $q(d)$. Thus there exists a nonzero vector $\left(b_{r}\right)$ such that $\sum c_{r s} b_{r}=0$ for all $s$. The homogeneous equation $\sum b_{i j k} x^{i} y^{j} z^{k}$ now describes a curve of degree $d$ passing through the $q(d)$ points.

Lemma 7.12. There exists a $T_{a}$-closed set $F \subseteq \mathbf{Q}^{d} \times \mathbf{Q}^{1} \times \mathbf{P}^{2} \times \mathbf{P}^{2}$ such that:

(i) If $e \in \mathbf{Q}^{d}, b \in \mathbf{Q}^{1}, a_{1}, a_{2} \in C_{d}(e) \cap C_{1}(b)$ and $a_{1}, a_{2}$ are distinct, then $\left(e, b, a_{1}, a_{2}\right) \in F$.

(ii) If $\left(e^{\prime}, b^{\prime}, a^{\prime}, a^{\prime}\right) \in F, e^{\prime}$ a generic point of $\mathbf{L}^{d}$, then $a^{\prime}$ is a double point of $C_{d}\left(e^{\prime}\right)$, or else $C_{1}\left(b^{\prime}\right)$ is one of the components of $C_{d}\left(e^{\prime}\right)$.

Proof. Let $F^{\prime}=\left\{\left(u, v, x_{1}, x_{2}\right): u \in \mathbf{Q}^{d}, v \in \mathbf{Q}^{1}, x_{1}, x_{2} \in C_{d}(u) \cap C_{1}(v)\right\}$, and let $F$ be the union of all components of $F^{\prime}$ not contained in the diagonal $x_{1}=x_{2}$. Then (i) holds. (Actually it is easy to see $F$ is irreducible.)

To show (ii), assume first that $\left(e^{*}, b^{*}, a_{1}^{*}, a_{2}^{*}\right) \in F$, where $\left(e^{*}, b^{*}\right)$ is a generic point of $\mathbf{L}^{d} \times \mathbf{Q}^{1}$; we claim $a_{1}^{*} \neq a_{2}^{*}$. Let $\left(e, b, a_{1}, a_{2}\right)$ be a generic point of $F$, specializing to $\left(e^{*}, b^{*}, a_{1}^{*}, a_{2}^{*}\right)$. Let $C_{d}(e) \cap C_{1}(b)=\left\{a_{1}, \ldots, a_{d}\right\}$. We can extend the specialization to a specialization $\left(e, b, a_{1}, \ldots, a_{d}\right) \rightarrow\left(e^{*}, b^{*}, a_{1}^{*}, \ldots, a_{d}^{*}\right)$. By 4.8 $C_{d}\left(e^{*}\right) \cap C_{1}\left(b^{*}\right)$ consists precisely of $\left\{a_{1}^{*}, \ldots, a_{d}^{*}\right\}$. But by genericity the points of intersection of $C_{1}\left(b^{*}\right)$ with the $d$ component lines of $C_{d}\left(e^{*}\right)$ must all be distinct, so $\left\{a_{1}^{*}, \ldots, a_{d}^{*}\right\}$ has size $d$. In particular $a_{1}^{*} \neq a_{2}^{*}$.

Now let $\left(e^{\prime}, b^{\prime}, a^{\prime}, a^{\prime}\right) \in F$, with $e^{\prime}$ a generic point of $\mathbf{L}^{d}$. Let $Y$ be the component of $F \cap\left(\mathbf{L}^{d} \times \mathbf{Q}^{1} \times \mathbf{P}^{2} \times \mathbf{P}^{2}\right)$ in which $\left(e^{\prime}, b^{\prime}, a^{\prime}, a^{\prime}\right)$ lies, and let $\left(e^{*}, b^{*}, a_{1}^{*}, a_{2}^{*}\right)$ be a generic point of $Y$. If $C_{1}\left(b^{*}\right)$ is one of the components of $C_{d}\left(e^{*}\right)$, then the corresponding fact holds true for $b^{\prime}, e^{\prime}$, so (ii) holds. Otherwise, $C_{1}\left(b^{*}\right) \cap C_{d}\left(e^{*}\right)$ is finite, so the projection of $Y$ to the first two coordinates is finite-to-one on some open set. Since $Y$ has codimension $\geq 4$ in $\left(\mathbf{L}^{d} \times \mathbf{Q}^{1} \times \mathbf{P}^{2} \times \mathbf{P}^{2}\right)$, by the dimension theorem, this forces $\left(e^{*}, b^{*}\right)$ to be a generic point of $\mathbf{L}^{d} \times \mathbf{Q}^{1}$. By the above, $a_{1}^{*} \neq a_{2}^{*}$. Let $L_{i}^{*}$ be a component line of $C_{d}\left(e^{*}\right)$ on which $a_{i}^{*}$ lies. We have a specialization $\left(e^{*}, b^{*}, a_{1}^{*}, a_{2}^{*}, L_{1}^{*}, L_{2}^{*}\right) \rightarrow\left(e^{\prime}, b^{\prime}, a^{\prime}, a^{\prime}, L_{1}^{\prime}, L_{2}^{\prime}\right)$. Since $e^{*}, e^{\prime}$ are generic in $\mathbf{L}^{d},\left(e^{*}, L_{1}^{*}, L_{2}^{*}\right) \rightarrow\left(e^{\prime}, L_{1}^{\prime}, L_{2}^{\prime}\right)$ is an isomorphism, and hence $L_{1}^{\prime} \neq L_{2}^{\prime}$. Thus $a^{\prime}$ lies on the two lines $L_{1}^{\prime}, L_{2}^{\prime}$, showing it is a double point of $C_{d}\left(e^{\prime}\right)$.

Definition. (i) A closed irreducible subset of $\mathbf{P}^{2}$ will be called a curve.

(ii) If $S \subseteq \mathbf{P}^{2}$ is a curve, then a generic line intersects $S$ in some definite number of points, which we call $\operatorname{deg}(S)$.

Lemma 7.13. For any $e \in \mathbf{Q}^{d}$ and any curve $S$, if $C_{d}(e) \cap S$ is finite, then it has size at most $d \cdot \operatorname{deg}(S)$.

Proof. If $S$ is a subset of an algebraic curve, then by 7.9 it is an algebraic curve, and this is standard; so we assume it is not. So $C_{d}(d)$ always meets $S$ in a finite set, and the exceptional set for the correspondence $C_{d} \cap\left(\mathbf{Q}^{d} \times S\right)$ on $\left(\mathbf{Q}^{d} \times S\right)$ is empty. Note that each irreducible component $T$ of $C_{d} \cap\left(\mathbf{Q}^{d} \times S\right)$ has codimension one in $C_{d}$, so $T(x)$ is nonempty for generic $x \in \mathbf{Q}^{d}$. Thus 4.8 is applicable here. 
((4.7A) certainly holds for any irreducible component of $C_{d} \cap\left(\mathbf{Q}^{d} \times S\right)$, since $S$ may be embedded in $\mathbf{P}^{2}$.)

Let $e^{*}$ be a generic point of $\mathbf{Q}^{d}$. Applying 4.8 to each component of $C_{d} \cap\left(\mathbf{Q}^{d} \times S\right)$, $\left|C_{d}(e) \cap S\right| \leq\left|C_{d}\left(e^{*}\right) \cap S\right|$. Hence we may assume $e$ is generic. Let $C_{d}(e) \cap S=$ $\left\{a_{1}, \ldots, a_{r}\right\}$ have size $r$. Let $b_{i j} \in \mathbf{Q}^{1}$ be the line passing through $a_{i}, a_{j}$. Let $u=\left(e, a_{1}, \ldots, a_{r}, b_{12}, \ldots, b_{r-1, r}\right)$, and let $H$ be the locus of $u$. By genericity of $e, H$ projects onto a dense subset of $\mathbf{Q}^{d}$; so by $7.3 H$ projects onto $\mathbf{Q}^{d}$. Let $e^{\prime} \in \mathbf{L}^{d}$ be generic. Then $\left(e^{\prime}, a_{1}^{\prime}, \ldots, b_{r-1, r}^{\prime}\right) \in H$ for some $a_{1}^{\prime}, \ldots, b_{r-1, r}^{\prime}$. Recall $F$ of 7.12 ; by 7.12(i) $\left(e, b_{i j}, a_{i}, a_{j}\right) \in F$ for all $i<j$, hence $\left(e^{\prime}, b_{i j}^{\prime}, a_{i}^{\prime}, a_{j}^{\prime}\right) \in F$. By $7.12($ ii) this implies that the points $a_{i}^{\prime}$ are distinct, unless $C_{1}\left(b_{i j}^{\prime}\right)$ is one of the components of $C_{d}\left(e^{\prime}\right)$.

However, $a_{i} a_{j} \rightarrow a_{i}^{\prime} a_{j}^{\prime}$ is a rank- 1 specialization $\left(\operatorname{rk}\left(a_{i}\right)=1\right.$ since $a_{i} \in S, a_{i}^{\prime} \notin$ $\operatorname{acl}(\emptyset)$ since $C_{d}\left(e^{\prime}\right)$ passes through $a_{i}^{\prime}$ and $e^{\prime}$ is generic). Thus it is not exceptional, so $\operatorname{rk}\left(b_{i j}^{\prime} / a_{i}^{\prime} a_{j}^{\prime}\right) \leq \operatorname{rk}\left(b_{i j} / a_{i}, a_{j}\right)=0$. Hence if $C_{1}\left(b_{i j}^{\prime}\right)$ is one of the components of $C_{d}\left(e^{\prime}\right)$, then $\operatorname{rk}\left(e^{\prime} / a_{i}^{\prime} a_{j}^{\prime}\right) \leq \operatorname{rk}\left(\mathbf{Q}^{d-1}\right)=2(d-1)$. But $\operatorname{rk}\left(e^{\prime}\right)=2 d$, so $\operatorname{rk}\left(a_{i}^{\prime} a_{j}^{\prime}\right)=2$, and in particular, they are in any case distinct.

Thus $C_{d}\left(e^{\prime}\right) \cap S$ has at least $r$ points. But $C_{d}\left(e^{\prime}\right)$ is just the union of $d$ generic lines, hence $\left|C_{d}\left(e^{\prime}\right) \cap S\right|=d \cdot \operatorname{deg}(S)$. This proves the required inequality.

Lemma 7.14. Every curve is algebraic.

Proof. Let $S$ be a curve. Choose $d$ such that $q(d)>d \cdot \operatorname{deg}(S)$. By 7.11 there exists $e \in \mathbf{Q}^{d}$ such that $C=C_{d}(e)$ meets $S$ in at least $q(d)$ points. By 7.13, $C \cap S$ must be infinite. By 7.6, $C \subseteq S$; since $S$ is irreducible, $C=S$.

Proof of 7.10. By [Hr4], 3.1, $T_{a}$ and $T_{b}$ have the same constructible sets. If $X$ is $T_{a}$-closed, it is $T_{b}$-closed. If $X$ is $T_{b}$-closed and irreducible, let $X^{*}$ be the $T_{a}$-closure of $X$. Clearly $X^{*}$ is $T_{a}$-irreducible. Now $X^{*}-X$ has Morley rank smaller than that of $X$, and this is a constructible notion. So $\operatorname{dim} X=\operatorname{dim} X^{*}$ in $T_{b}$. But by $7.14 X^{*}$ is irreducible in $T_{b}$, so $X=X^{*}$. Thus the two topologies coincide.

\section{TheOrem B}

In this section we prove Theorem B and Proposition 1.1.

Theorem D. Let $D$ be an ample Zariski geometry. Then there exists an algebraically closed field $K$ and a surjective Zariski map $f: D \rightarrow \mathbf{P}^{1}(K)$. $f$ maps constructible sets to (algebraically) constructible sets, and in fact is a closed Zariski map on $D-F$ for some finite $F$.

Remark 8.1. (a) From a model-theoretic point of view, $K$ is interpretable in $D$, and the induced structure is the pure field structure (with a subfield of distinguished constants).

(b) One may show for strongly minimal sets $D$ in general: if the statement of (a) holds, then the field $K$ of (a) can be interpreted without parameters. This gives a canonicity statement which is trivial here, since the language names constants for a model. However a more satisfying canonicity statement will be obtained later (Theorem $\mathrm{B}^{\prime}$ ).

Proof of Theorem B. By $6.11 D$ interprets a one-dimensional field $K$. By 5.11 and $5.13 \mathrm{~K}$ may be given a manifold structure, so that the field operations are morphisms. By Macintyre's theorem [Ma], $K$ is algebraically closed. (This may 
also be deduced here as follows. If $f$ is a nonconstant polynomial, then $f$ describes a morphism from $K$ to $K$, carrying $z$ to $f(z)$. $f$ extends to a morphism $g$ from $\mathbf{P}^{1}$ to $\mathbf{P}^{1}$, by taking the closure of its graph. By $7.1 \mathrm{~g}$ is surjective, and so attains the values 0 and $\infty$. Since $f$ does not take the value $\infty$, we must have $g(\infty)=\infty$, so $g(a)=0$ for some $a \neq \infty$, hence $f(a)=0$ and $f$ has a root.)

Since $K$ is interpreted in $D$, if $a$ is a generic point of $K$, then

$$
a \in \operatorname{acl}\left(d_{1}, \ldots, d_{k}\right) \text { for some } d_{1}, \ldots, d_{k} \in D \text {. }
$$

Minimizing $k$ and working over $\left\{d_{1}, \ldots, d_{k-1}\right\}$, we find generic points $a, b$ of $K, D$ respectively, such that $\operatorname{acl}(a)=\operatorname{acl}(b)$.

Lemma 8.2. There exists a nonconstant morphism $D \rightarrow \mathbf{P}^{1}$.

Proof. Let $a, b$ be generic points of $D, K$ respectively with $\operatorname{acl}(a)=\operatorname{acl}(b)$. Let $C(a)$ be the locus of $b$ over $a$. Then $C(a)$ is a finite subset of $K$, hence is the set of roots of a unique monic polynomial $\sum b_{i} x^{i} . C(a)$ is left invariant by automorphisms fixing $a$, hence so is each coefficient $b_{i}$; thus $b_{i} \in \operatorname{dcl}(a)$. We have $b \in \operatorname{acl}\left(b_{0}, \ldots, b_{n-1}\right)$ and so not all the elements $b_{i}$ can be in $\operatorname{acl}(\emptyset)$. Pick one of the coefficients, $b^{\prime}$, which is not in $\operatorname{acl}(\emptyset)$. Let $H$ be the locus of $\left(a, b^{\prime}\right)$ over $\operatorname{acl}(\emptyset)$. Then $H$ is a closed, irreducible set of dimension 1. For generic $a \in D, H(a)$ has a single point. Hence by 4.13 and 4.8 , for all $a^{\prime} \in D, H\left(a^{\prime}\right)$ has at most one point, or else some $H\left(a^{\prime}\right)$ is infinite. The infinite case is impossible, since $a^{\prime} \times K$ would then be a component of $H$, but $H$ is irreducible. So $H$ is the graph of a partial function. By 7.1 it is total, and hence a morphism $D \rightarrow \mathbf{P}^{1}$.

Claim. There exists a surjective morphism $D \rightarrow \mathbf{P}^{1}$.

Proof. Let $h: D \rightarrow \mathbf{P}^{1}$ be a nonconstant morphism. Composing with a linear fractional transformation, we may assume the points $0, \infty$ are in the range $h D$ of $h$. Let $n$ be larger than $\left|\mathbf{P}^{1}-h D\right|$, and prime to the characteristic. Every element of $\mathbf{P}^{1}-\{0, \infty\}$ has $n$ distinct $n$th roots, and it follows that for every $a \in \mathbf{P}^{1}$, some $n$th root of $a$ is in the range. Thus composing $h$ with the $n$th power map yields a surjective morphism.

Lemma 8.3. Every surjective morphism $h: D \rightarrow \mathbf{P}^{1}$ is a Zariski-closed map on some cofinite subset of $D$.

Proof. Let $b \in \mathbf{P}^{1}$ be generic, and let $h^{-1}(b)=\left\{a_{1}, \ldots, a_{m}\right\}$. Let $F^{*}$ be the locus of $\left(b,\left(a_{1}, \ldots, a_{m}\right)\right)$ over $\operatorname{acl}(\emptyset)$. We will find an "inverse morphism" to $h$, from a cofinite subset of $\mathbf{P}^{1}$ into the special sort $[D]^{m}$. Recall $[D]^{m}$ is defined as the quotient of $D^{m}$ by $\operatorname{Sym}(m)$; let $q: D^{m} \rightarrow[D]^{m}$ be the quotient map. Then $F=q F^{*}$ is a closed, irreducible subset of $\mathbf{P}^{1} \times[D]^{m}$. For generic $b \in \mathbf{P}^{1}, F(b)$ consists of a single point. (If $\left(b,\left(a_{1}^{\prime}, \ldots, a_{m}^{\prime}\right)\right) \in F^{*}$, then $a_{1}^{\prime}, \ldots, a_{m}^{\prime}$ are distinct elements of $h^{-1}(b)$, so $q\left(a_{1}^{\prime}, \ldots, a_{m}^{\prime}\right)=q\left(a_{1}, \ldots, a_{m}\right)$.) Thus as in 8.2 , since $\operatorname{dim}(F)=1$, $F$ is the graph of a partial function $f$, with domain $Y \subseteq \mathbf{P}^{1}$. Let $Z=h^{-1} Y$, a cofinite subset of $D$. Then $h$ is closed on $Z$. For if $C \subseteq Z^{k}$ is a closed set, then so is $C \#=\left\{\left(\left(a_{1}^{1}, \ldots, a_{m}^{1}\right), \ldots,\left(a_{1}^{k}, \ldots, a_{m}^{k}\right)\right) \in\left(D^{m}\right)^{\bar{k}}\right.$ : for some function $\left.\nu:\{1, \ldots, k\} \rightarrow\{1, \ldots, m\},\left(a_{\nu 1}^{1}, \ldots, a_{\nu k}^{k}\right) \in C\right\}$. Hence $q C \#$ is also closed. Now $S=\left\{s \in[D]^{m}: h\right.$ is constant on $\left.s\right\}$ is a closed subset of $[D]^{m}$, and $f(b) \in S$ for generic $b$, so $f(b) \in S$ for all $b$. Thus $h C=f^{-1}(q C \#)$ and so $h C$ is closed.

This finishes the proof of Theorem B.

Using 7.4, it seems 8.3 could be stated for an arbitrary algebraic curve rather than $\mathbf{P}^{1}$, improving Theorem $\mathrm{B}^{\prime}$. 
Remark 8.4. Lemma 8.3 probably cannot be improved to " $h$ is Zariski-closed". For example one may take a cover $h: D \rightarrow \mathbf{P}^{1}$ where $h^{-1}(a)$ has two points except for $a=0, h^{-1}(0)$ has a single point, and the closed sets are generated by the graphs of equality, the set $B=\{(x, y): h x=h y, x \neq y\}$, and the inverse images under $h$ of the closed subsets of $\mathbf{P}^{1}$.

We now recall and prove:

Proposition 1.1. Let $C$ be a smooth curve over an algebraically closed field $F$, and $C^{\prime}$ a smooth curve over a field $F^{\prime}$. Suppose $h: C \rightarrow C^{\prime}$ is an isomorphism of Zariski geometries. (The induced map on $C^{n}$ is a homeomorphism for each n.) Then there exists an isomorphism of fields $h_{F}: F \rightarrow F^{\prime}$, such that $h$ is an isomorphism of algebraic varieties with respect to the identification of fields $h_{F}$.

Proof. Let $K$ be a field interpretable in the Zariski geometry $C$; in the present case this can be done explicitly, since $C$ is given as an algebraic curve, and one sees directly that $K$ is isomorphic to $F$ by an $F$-definable map $g: F \rightarrow K$. Viewing $K$ as a $C$-manifold as in $\S 5$, it becomes also an $F$-manifold, and by $5.12 \mathrm{~g}$ is a morphism of manifolds. The isomorphism $h: C \rightarrow C^{\prime}$ extends to an isomorphism between $(C, K)$ and $\left(C^{\prime}, K^{\prime}\right)$ for some $K^{\prime}$ interpretable over $C^{\prime}$. Now since $K^{\prime}$ is interpretable over $C^{\prime}$ and $C^{\prime}$ over $F^{\prime}, K^{\prime}$ is also interpretable over $F^{\prime}$, perhaps by a different formula than the one interpreting $K$ over $F$. At all events (by $[\mathrm{Po}])$ there exists an $F^{\prime}$-definable field isomorphism $g^{\prime}: F^{\prime} \rightarrow K^{\prime}$. Let $p$ be the characteristic exponent, and let $\operatorname{Fr}(x)=x^{p}$ be the Frobenius map (on any of the fields involved). Extend $h$ to $F$ by the formula: $g^{\prime} h_{F}=\operatorname{Fr}^{n} h_{K} g$, where $n$ is an integer to be determined later. We view $h=\left(h_{F}, h_{C}, h_{K}\right)$ as a map from $(F, C, K)$ to $\left(F^{\prime}, C^{\prime}, K^{\prime}\right)$.

Let $F(C)$ be the field of rational functions on $C$ (considered as functions from $C$ to the projective line $\left.P^{1}(F)=F \cup\{\infty\}\right)$, and let $F_{p}(C)$ be its perfect closure, the field of all functions of the form $h(x)^{1 / q}$, where $h \in F(C)$ and $q$ is a power of $p$. Let $F_{p}^{\prime}\left(C^{\prime}\right)$ be defined analogously.

Claim. $h$ carries $F_{p}(C)$ to $F_{p}^{\prime}\left(C^{\prime}\right)$.

Proof. We use the following characterization of $F_{p}(C)$ : a function $c: C \rightarrow P^{1}(F)$ is in $F_{p}(C)$ if and only if it is not constantly $\infty$, and the graph of $c$ is a closed irreducible subset of $C \times P^{1}(F)$.

Let $c: C \rightarrow P^{1}(F) \in F_{p}(C)$. Then the graph of $c$ in $C \times P^{1}(F)$ is Zariski closed and irreducible. Since $g$ is a morphism, the same is true of the graph $\Gamma$ of $g \circ c . h$ preserves the Zariski topologies, and hence $h(\Gamma)$ is closed irreducible. Now $h(\Gamma)$ is the graph of $\mathrm{Fr}^{-n} \circ g^{\prime} \circ h(c)$. Since Fr and $g^{\prime}$ are morphisms (of Zariski geometries), the graph of $h(c)$ is closed irreducible, and so $h(c) \in F_{p}^{\prime}\left(C^{\prime}\right)$.

Claim. $h$ carries $F(C)$ to $\operatorname{Fr}^{m}\left(F^{\prime}\left(C^{\prime}\right)\right)$, for some $m$.

Proof. Let $X$ be the collection of subfields of $F^{\prime}\left(C^{\prime}\right)$ containing $F^{\prime}$, whose perfect closure is $F_{p}^{\prime}\left(C^{\prime}\right)$. If $L, L^{\prime} \in X$, then we may choose $x \in L, x^{\prime} \in L^{\prime}$ such that $L$ is separable over $F(x)$, and $L^{\prime}$ over $F\left(x^{\prime}\right)$. For some $m$, we have $p\left(\operatorname{Fr}^{m}(x), x^{\prime}\right)=0$, where $p$ is a nonzero polynomial, separable in both variables. Thus $\operatorname{Fr}^{m}(L)$ is separable over $L^{\prime}$, and $L^{\prime}$ over $\operatorname{Fr}^{m}(L)$; so $\operatorname{Fr}^{m}(L)=L^{\prime}$. We have shown that the elements of $X$ form a single orbit under the action of Frobenius. Since $h$ clearly carries $X$ to the correspondingly defined $X^{\prime}$, the Claim follows.

Thus with an appropriate choice of the integer $n$, we obtain $h$ such that $h$ carries $F(C)$ to $F^{\prime}\left(C^{\prime}\right)$. If we use $h$ to identify $F$ and $F^{\prime}$, then $h_{C}$ is a bijection 
carrying rational functions to rational functions, and hence is an isomorphism of varieties.

\section{INTERNAL AND EXTERNAL COVERS}

Towards the proof of Theorem A, we require some analysis of covers; up to Proposition 9.6 we work in the constructible category. These lemmas are valid in any omega-stable structure (or any stable structure, if one replaces types by *-types, and finite sets by countable ones).

Definition. Let $P, Q$ be types over a finite set $A$, and let $f: P \rightarrow Q$ be an $A$ definable map. Also let $R$ be a set. $f: P \rightarrow Q$ is called $R$-external if for any finite $B$ containing $A$, and any $a, a^{\prime}$ in $P$ independent from $B$ over $A$, if $f(a)=f\left(a^{\prime}\right)=c$, then $a, a^{\prime}$ are conjugate over $R \cup B \cup\{c\}$.

A type $Q$ over $A$ is $R$-internal if for some finite $B$ containing $A$, no two distinct points of $Q$ are conjugate over $R$.

In general, we will omit $A$ from the notation.

Remark 9.1. The notion of $R$-internal was introduced in [Hr7], in a different formulation (for $Q$ a type over an algebraically closed set).

(*) For some $B$ and some $a \in Q$ independent from $B, a \in \operatorname{dcl}(B \cup R)$.

We show the equivalence of the two versions. If $(*)$ holds, let $B^{*}$ be a set such that for any conjugate $B^{\prime}$ of $B$ over $A, B^{*}$ contains a conjugate of $B^{\prime}$ over acl $(A)$. $B^{*}$ can be chosen finite, by omega-stability. Let $B \#$ contain enough independent copies of $B^{*}$, so that any element of $Q$ is independent over $A$ from some copy of $B^{*}$ inside $B \#$. Now any $a^{\prime} \in Q$ is conjugate to $a$ over $A$, and hence for some $B^{\prime}$ conjugate to $B$ over $A$, and independent from $a$ over $A, a^{\prime} \in \operatorname{dcl}\left(B^{\prime \prime} \cup R\right)$. Thus for any $B^{\prime \prime}$ conjugate to $B^{\prime}$ over $\operatorname{acl}(A)$, and independent from $a$ over $A$, $a^{\prime} \in \operatorname{dcl}\left(B^{\prime} \cup R\right)$. Since such a $B^{\prime \prime}$ exists inside $B \#$, we have $a^{\prime} \in \operatorname{dcl}(B \# \cup R)$. We have shown that $(*)$ is equivalent to:

$(* *)$ For some finite $B, Q \subseteq \operatorname{dcl}(B, R)$.

Clearly $(* *)$ implies our definition of $R$-internal; the converse follows from 3.3 and 3.4. Thus the three definitions coincide.

Lemma 9.2. Let $P$ be a type over the finite set $A$, and let $R$ be an $A$-definable set. Then there exists a type $P^{*}$ over $A$ (consisting perhaps of imaginary elements), and a definable map $f: P \rightarrow P^{*}$, such that:

(i) $P^{*}$ is $R$-internal.

(ii) $f: P \rightarrow P^{*}$ is $R$-external.

Proof. We assume notationally $A=\emptyset$. By omega-stability, there exists $a^{*} \in \operatorname{dcl}(a)$ such that:

(i) $a^{*} \in P^{*}$, an $R$-internal type over $A$.

(ii) If $b \in \operatorname{dcl}(a)$ and is in an $R$-internal type over $A$, then $b \in \operatorname{dcl}\left(a^{*}\right)$.

We may write $a^{*}=f(a)$, where $f: P \rightarrow P^{*}$ is a definable function. We must show $f: P \rightarrow P^{*}$ is $R$-external. Consider the equivalence relations $E_{0}, E, E^{*}$ on $P$ defined as follows:

$a E_{0} a^{\prime}$ if $f(a)=f\left(a^{\prime}\right)$;

$a E a^{\prime}$ if $f(a)=f\left(a^{\prime}\right)$ and for any $B$ independent from $\left\{a, a^{\prime}\right\}, a, a^{\prime}$ are conjugate over $B \cup R \cup f(a)$;

$a E^{*} a^{\prime}$ if $f(a)=f\left(a^{\prime}\right)$, and $a, a^{\prime}$ are conjugate over $\operatorname{acl}(f(a))$. 
By omega-stability, $E^{*}$ is a definable equivalence relation, refining each $E_{0}$-class into a finite number of classes.

By [Hr7], Proposition 2.4, for any $B$ independent from $a, a$ is independent from $B \cup R$ over $f(a)$. Thus if $f(a)=f\left(a^{\prime}\right)$ and $\operatorname{tp}(a / \operatorname{acl}(f(a)))=\operatorname{tp}\left(a^{\prime} / \operatorname{acl}\left(f\left(a^{\prime}\right)\right)\right)$, then $a, a^{\prime}$ are conjugate over $B \cup R \cup\{f(a)\}$. So if $a E^{*} a^{\prime}$, then $a E a^{\prime}$. Thus $E^{*} \subseteq E \subseteq E_{0}$. It follows that $E$ is a definable equivalence relation, refining each $E_{0}$-class into a finite number of classes.

Let $f \#$ be such that $f \#(a)=f \#\left(a^{\prime}\right)$ iff $a E a^{\prime}$. Let $P \#$ be the type of $f \#(a), a \in$ $P$. Then $P \#$ is $R$-internal. To see this note first that since $E$ refines $E_{0}$, we have $f=g f \#$ for some $g$. Fix $a \# \in P \#, b=g(a \#)$. One can choose a finite $B$ such that $P^{*} \subseteq \operatorname{dcl}(B, R), b$ is independent from $B$, and if $g(c)=b, c \neq a \#$, then $c, a \#$ are not conjugate over $B \cup\{b\} \cup R$. (There are finitely many possible choices of $c$, and for each one such a $B$ exists.) But then $b \in P^{*} \subseteq \operatorname{dcl}(B, R), g^{-1}(b) \subseteq \operatorname{acl}(b)$, and no two points of $g^{-1}(b)$ are conjugate over $B \cup\{b\} \cup R$, so $g^{-1}(b) \subseteq \operatorname{dcl}(B, R)$, and in particular $a \# \in \operatorname{dcl}(B, R)$. Thus $P \#$ is $R$-internal. By (ii), $f \#(a) \in \operatorname{dcl}(f(a))$. It follows that $g$ is $1-1$, and $E=E_{0}$. This proves that $f$ is $R$-external, as required.

Remark 9.3. Let $f: P \rightarrow P^{*}$ be as above: $P^{*}$ is $R$-internal, and $f: P \rightarrow P^{*}$ is $R$-external. Let $Q$ be any $R$-internal type over $A$, and $g: P \rightarrow Q$ any $A$-definable map. Then there exists an $A$-definable map $h: P^{*} \rightarrow Q$ such that $g=h f$. In particular, if $g$ is finite-to-one, then so is $f$.

Proof. We must show that if $f(a)=f\left(a^{\prime}\right)$, then $g(a)=g\left(a^{\prime}\right)$. Suppose not. For some $B$ independent from $a, a^{\prime}$ over $A$, we have $Q \subseteq \operatorname{dcl}(B \cup R)$. Now $a, a^{\prime}$ are conjugate over $B \cup R$; so $g(a), g\left(a^{\prime}\right)$ are conjugate over $B \cup R$; since $g(a), g\left(a^{\prime}\right)$ are in $\operatorname{dcl}(B \cup R)$, they must be equal.

Lemma 9.4. Let $f: P \rightarrow Q$ be R-external (over $A$ ), $c \in Q, b \in P, f(b)=c$. Let $B$ be independent from $b$ over $A$. Then $b$ is independent from $R \cup B \cup\{c\}$ over $A \cup\{c\}$.

Proof. It suffices to show that for any finite $R_{0} \subseteq R, b$ is independent from $R_{0} \cup$ $B \cup\{c\}$ over $A$. This is clear since there exists some $b^{\prime} \in f^{-1}(c)$ independent from $R_{0} \cup B \cup\{c\}$ over $A \cup\{c\}$, and any such $b^{\prime}$ is conjugate to $b$ over $R_{0} \cup B \cup\{c\}$.

Notation. If $P$ is a type over some set $A$, let $a_{1}, \ldots, a_{n}$ be elements of $P$ having the same type over $\operatorname{acl}(A)$, and independent over $\operatorname{acl}(A)$. Then the type of $\left(a_{1}, \ldots, a_{n}\right)$ is determined (3.8). We denote it by $[P]^{k}$.

Lemma 9.5. Let $f: P \rightarrow Q$ be R-external, and let $f^{[n]}$ denote the induced map from $P^{[n]}$ to $Q^{[n]}$. Then $f^{[n]}$ is R-external.

Proof. For notational convenience we prove the case $n=2$. Let $B$ contain $A, a=$ $\left(a_{1}, a_{2}\right) \in P^{[2]}$, with $a$ independent from $B$ over $A$. We must show that $\operatorname{tp}(a / B \cup R)$ depends only on $c=\left(c_{1}, c_{2}\right)=f^{[2]}(a) \in Q^{[2]}$. Indeed $\operatorname{tp}\left(a_{2} / B \cup R \cup\left\{c_{2}\right\}\right)$ is determined by $c_{2}$, since $f$ is external. Letting $B^{\prime}=B \cup\left\{a_{2}\right\}$, we have that $a_{1}$ is independent from $B^{\prime}$ over $A$, and $\operatorname{tp}\left(a_{1} / B^{\prime}\right)$ is determined by $c_{1}$. Together these facts show that $\operatorname{tp}(a / B \cup R)$ is determined by $c$.

Proposition 9.6. Let $P, P^{*}, E, C$ be complete types over $A, f: P \rightarrow P^{*} R$-external. Suppose $E \subseteq \operatorname{acl}(R), C \subseteq E \times P$, and $C(e)$ is strongly minimal for $e \in E$. Further suppose ("ampleness") $[P]^{2} \cap C(e)^{2} \neq \emptyset$ for $e \in E$. Then either $f$ is 
constant on each $C(e)$, or else $f$ is finite-to-one, and $C(e)=f^{-1} f C(e)$ for each $e \in E$.

Proof. Let $e \in E$, and suppose $f$ is not constant on $C(e)$. Then the equivalence relation $f(x)=f(y)$ has more than one class on $C(e)$. Since $C(e)$ is strongly minimal, it has at most one infinite class. Since $C(e)$ forms a complete type over $e$, if there does exist an infinite class, then every element of $C(e)$ belongs to this class; so there is only one class, contradicting our assumption. Thus all classes of the equivalence relation are finite, so $f$ is finite-to-one on $C(e)$. In particular the image $f C(e)$ of $C(e)$ under $f$ is strongly minimal.

From strong minimality, it follows that for some integer $N$, whenever $e \neq e^{\prime}$ we have $\left|C(e) \cap C\left(e^{\prime}\right)\right| \leq N$, or else $\left|C(e)-C\left(e^{\prime}\right)\right| \leq N$ and $\left|C\left(e^{\prime}\right)-C(e)\right| \leq N$. Define $e \sim e^{\prime}$ if the latter possibility holds. Let $E^{*}=E / \sim$, and write $e^{*}$ for $e / \sim$. Then there exists a type $C^{*} \subseteq E^{*} \times P$ such that for any $e \in E$, the two sets $C(e), C^{*}\left(e^{*}\right)$ agree except perhaps for finitely many points (3.9). But all points of $C(e)$ have the same type over $e$, so $C(e) \subseteq C^{*}\left(e^{*}\right)$. Thus the "ampleness" hypothesis holds for $C^{*}$. Since $f$ is finite-to-one on $C(e)$, it is also finite-to-one on $C^{*}\left(e^{*}\right)$. If we succeed in showing that $C^{*}\left(e^{*}\right)=f^{-1} C\left(e^{*}\right)$, then $f^{-1}(f b) \subseteq C(e)$ for almost all $b \in C(e)$; since all points of $C(e)$ have the same type, it follows that $f^{-1}(f b) \subseteq C(e)$ for all $b \in C(e)$, so that $C(e)=f^{-1} f C(e)$. Thus we may replace $C$ by $C^{*}$; in other words we may assume that if $e \neq e^{\prime}$, then $\left|C(e) \cap C\left(e^{\prime}\right)\right| \leq N$.

Let $\beta$ be the equivalence relation on $E$ defined by: $e \beta e^{\prime}$ iff $\operatorname{tp}(e / R)=\operatorname{tp}\left(e^{\prime} / R\right)$. By assumption, each $\beta$-class is finite. Let $\beta e$ denote the $\beta$-class of $e$. Then $f C(e)$ is determined by $\beta e$. Moreover $\beta E \subseteq \operatorname{dcl}(R)$. Further we have $e \in \operatorname{dcl}(\beta e, a)$, where $a$ is any generic point of $C(e)$. Indeed let $e \#=\beta e$; then $e$ is the unique element of $E$ satisfying: $\beta e=e \#, a \in C(e)$. For if $\beta e^{\prime}=e \#$ and $a \in C\left(e^{\prime}\right)$, while $e \neq e^{\prime}$, then $C(e) \cap C\left(e^{\prime}\right)$ is finite, so $a \in \operatorname{acl}\left(e, e^{\prime}\right)=\operatorname{acl}(e \#)$, contradicting the genericity of $a$.

Since $f$ is $R$-external, $\operatorname{tp}(a / f(a))$ implies $\operatorname{tp}(a / f(a), \beta e)$. Since $a \in \operatorname{acl}(e, f(a))=$ $\operatorname{acl}(\beta e, f(a))$ when $a \in C(e)$, it follows that $a \in \operatorname{acl}(f(a))$. Thus $f$ is finite-to-one.

In particular, $f^{-1} f C(e)$ has Morley rank 1 , and can only contain finitely many curves $C\left(e^{\prime}\right)$. Let $\left(b, b^{\prime}\right) \in[P]^{2}, b, b^{\prime} \in C(e)$. Let $b^{\prime \prime}$ be any point such that $f\left(b^{\prime \prime}\right)=f\left(b^{\prime}\right), b^{\prime \prime} \notin C(e)$. Then $\operatorname{acl}\left(b^{\prime}\right)=\operatorname{acl}\left(b^{\prime \prime}\right)$, so $\left(b, b^{\prime \prime}\right) \in[P]^{2}$. By 9.5, $\operatorname{tp}\left(b, b^{\prime} / \beta e\right)=\operatorname{tp}\left(b, b^{\prime \prime} / \beta e\right)$, hence $\operatorname{tp}\left(b^{\prime} / b, \beta e\right)=\operatorname{tp}\left(b^{\prime \prime} / b, \beta e\right)$, and in particular $\operatorname{tp}\left(b^{\prime} / e\right)=\operatorname{tp}\left(b^{\prime \prime} / e\right)$. Thus $b^{\prime \prime} \in C(e)$. Now $b^{\prime \prime}$ was an arbitrary point of $f^{-1} f\left(b^{\prime}\right)$, so $f^{-1} f\left(b^{\prime}\right) \subseteq C(e)$; since all points of $C(e)$ have the same type as $b^{\prime}$ over $e$, $f^{-1} f C(e) \subseteq \bar{C}(e)$.

Now we use the following idea: if we have a family of "curves" separating points on $P$, each such "curve" is determined by its projection to $P^{*}$ by 9.6 , so the set of curves is $R$-internal. If the set of curves separates points on $P$, then $P$ is $R$-internal. However, we need a lemma to bridge a gap between the set of curves used here, indexed by a type, and in the original definition of very ampleness, where the index set is a closed set. The separation of points condition in that definition states that for any $a, b \in D^{2}$ there exists $e \in E$ such that $C(e)$ passes through just one of $a, b$. Let $E^{*}$ be the type associated with $E$. We need to know that for any distinct generic $a, b \in D^{2}$ there exists $e \in E^{*}$ such that $C(e)$ passes through just one of $a, b$.

Lemma 9.7. Let $C \subseteq X \times Y$ be closed irreducible, $X \subseteq D^{m}, Y \subseteq D^{n}$, and suppose $C$ projects onto a dense subset of $X$ and of $Y$. Let $F$ be a proper closed subset of $Y$. For generic $a \in X, C(a)$ can have no component contained in $F$. 
Proof. Suppose otherwise. Let $a$ be a generic element of $X$, and let $U$ be the union of all components of $C(a)$ not contained in $F$. Then $U$ is a closed set, invariant under automorphisms fixing $a$, so by $4.3 U$ has the form $C^{*}(a)$ for some closed $C^{*} \subseteq$ $D^{m} \times D^{n}$. We may replace $C^{*}$ by $C^{*} \cap C$. Then for generic $a, C(a) \subseteq C^{*}(a) \cup F$. Hence $C \subseteq C^{*} \cup(X \times F) \cup\left(F^{\prime} \times Y\right)$ for some proper closed $F^{\prime} \subseteq X$. Since $C$ is irreducible, it is contained in one of the three closed sets; it projects densely to $X$ and to $Y$, so $C^{*}$ is the only possibility. Thus $C(a)=C^{*}(a)=U$, so no component of $C(a)$ is contained in $F$.

Lemma 9.8. Let $C \subseteq Y \times E$ be closed irreducible. Assume $E$ is closed irreducible, 0 -definable, with associated type $E^{*}$. Suppose $C(a) \neq C(b)$ for distinct generic points $a, b \in Y$. Then $C(a) \cap E^{*} \neq C(b) \cap E^{*}$ for distinct generic points $a \neq b \in Y^{2}$.

Proof. Otherwise there is some 0-closed set $F$ in $E$ such that $C(a)-F=C(b)-F$. So $C(a) \subseteq C(b) \cup F$. But by 9.7 , no component of $C(a)$ lies in $F$. Thus all components of $C(a)$ are contained in $C(b)$, so $C(a) \subseteq C(b)$. Similarly $C(b) \subseteq C(a)$, so they are equal, a contradiction.

Lemma 9.9. Let $\operatorname{dim}(Y)=2, Y$ irreducible. Let $C \subseteq E \times Y$ be a generically normal family of curves on $Y: C(e)$ is closed irreducible and one-dimensional for generic $e \in E$, and if $e, e^{\prime}$ are distinct generic points, then $C(e) \neq C\left(e^{\prime}\right)$. Assume that for generic $\left(y_{1}, y_{2}\right) \in Y$ there exists $e \in E$ with $y_{1}, y_{2} \in C(e)$. Then for generic $\left(y_{1}, y_{2}\right) \in Y$ there exists $e \in E^{*}$ with $y_{1}, y_{2} \in C(e)$.

Proof. The assumption implies $\operatorname{dim}(E) \geq 2$. For if $\operatorname{dim}(E)=1, y_{1}, y_{2} \in C(e)$, then $e \in \operatorname{acl}\left(y_{1}\right), \operatorname{so} \operatorname{rk}\left(y_{2}\right)=\operatorname{rk}\left(y_{2} / y_{1}\right) \leq \operatorname{rk}\left(y_{2} / e\right) \leq 1$, a contradiction. Thus $\operatorname{rk}\left(E^{*}\right) \geq 2$. Suppose for generic $\left(y_{1}, y_{2}\right) \in Y$ there is no $e \in E^{*}$ with $y_{1}, y_{2} \in C(e)$. Then for $e \in E^{*}$ and a generic $y_{1} \in C(e)$, all points $y_{2} \in C(e)$ lie on some proper $y_{2}$-closed subset of $Y$. This subset is one-dimensional, hence contains only finitely many distinct sets $C\left(e^{\prime}\right)$. So $e \in \operatorname{acl}\left(y_{2}\right)$. Thus $\operatorname{rk}(e)+\operatorname{rk}(y / e)=\operatorname{rk}(y), \operatorname{so} \operatorname{rk}(e) \leq 1$, a contradiction.

We recall the statement of Theorem A.

Theorem A. Let $X$ be a very ample Zariski geometry. Then there exists a smooth curve $D$ over an algebraically closed field $K$, such that $X, D$ are isomorphic as Zariski geometries.

Proof. Let $X$ be a very ample Zariski geometry. Let $C \subseteq E \times X^{2}$ demonstrate that $X$ is very ample. Since $X$ is ample, by $6.11,5.11,5.13$ there exists an $X$ manifold $K$ with a field structure. Let $E^{*}$ be the type associated with the closed irreducible set $E$. Using $9.8,9.9$ we can strengthen (ii) of the definition of very ample to

(ii') For any distinct generic points $a \neq b \in X^{2}$, there exists $e \in E^{*}$ such that $C(e)$ passes through just one of $a, b$.

We also have: Replacing $E$ by another manifold if necessary, using the results of $\S 5$ to factor out the equivalence relation $C(e)=C\left(e^{\prime}\right)$, we may assume

(iii) If $e \neq e^{\prime} \in E^{*}$, then $C(e) \neq C\left(e^{\prime}\right)$.

By 9.2 there exists a $K$-external map $f: X \rightarrow P, P K$-internal. By $9.5 f^{[2]}: X^{[2]}$ $\rightarrow P^{[2]}$ is $K$-external. Let $C^{*}$ be the type associated with $C \subseteq E \times X^{2}$. It is easy to see that $C^{*} \subseteq E^{*} \times[X]^{2}$. Moreover for $e \in E^{*}$, the curve $C(e)$ is the closure of $C^{*}(e)$. By 9.6, if $e \in E^{*}$, then $C^{*}(e)=f^{-1} f C^{*}(e)$. Hence if $e \neq e^{\prime} \in E^{*}$, then by 
(iii), $C(e) \neq C\left(e^{\prime}\right)$, so $C^{*}(e) \neq C^{*}\left(e^{\prime}\right)$, hence $f C^{*}(e) \neq f C^{*}\left(e^{\prime}\right)$. It follows that $e, e^{\prime}$ are not conjugate over $P$ : if $\sigma$ is an automorphism fixing $P$, then since $f C^{*}(e) \subseteq P$ we have $f C^{*}(\sigma e)=\sigma f C^{*}(e)=f C^{*}(e)$. Thus $E^{*} \subseteq \operatorname{dcl}(P)$, so $E^{*}$ is $K$-internal. Now let $a \neq b \in X^{2}$; by (ii') there exists, say, $e \in E^{*}$ such that $a \in C(e)$ and $b \notin C(e)$; thus if $\sigma$ is an automorphism fixing $P$, then $\sigma e=e$, so $\sigma a \neq b$. Hence any automorphism fixing $P$ must also fix $X^{2}$ pointwise. So $X \subseteq \operatorname{dcl}(P)$ is $K$-internal.

By 9.1(*), working over appropriate parameters, a generic element $a \in X$ satisfies $a \in \operatorname{dcl}(b)$ for some $b \in K^{n}$. Thus $a=h(b)$ for some 0-definable function $h$. Define $b E b^{\prime}$ if $h(b)=h\left(b^{\prime}\right)$. Then $E \subseteq K^{2 n}$ is a constructible set in the sense of $X$, hence also in the sense of $K$ (Proposition 7.10). The imaginary element $b^{\prime}=b / E$ satisfies: $\operatorname{dcl}\left(b^{\prime}\right)=\operatorname{dcl}(a)$. Using elimination of imaginaries 5.1 applied to $K$, there exists a $K$-manifold $V$ (hence an algebraic variety) and an element $b^{\prime \prime}$ of $V$ such that $\operatorname{dcl}\left(b^{\prime}\right)=\operatorname{dcl}\left(b^{\prime \prime}\right)$. Note that $\operatorname{rk}\left(b^{\prime \prime}\right)=\operatorname{rk}(a)=1$. Replacing $V$ by the locus of $b^{\prime \prime}$ over $\operatorname{acl}(\emptyset)$, we may assume $\operatorname{dim}(V)=1$, and $V$ is irreducible. Further replacing $V$ by its normalization, we may assume it is a smooth, complete curve over $K$. Let $H$ be the locus of $\left(a, b^{\prime \prime}\right)$ over $\operatorname{acl}(\emptyset)$. Then $H$ is a closed irreducible subset of $X \times V$, $\operatorname{dim}(H)=1$, and for generic $x \in X$ there exists a unique $v \in V$ with $(x, v) \in H$, and vice versa. By 4.13 and 4.8, for each $x \in X$ there exists at most one $v \in V$ with $(x, v) \in H$, and vice versa. $V$ may be embedded in $\mathbf{P}^{3}$, so $H$ may be seen as a subset of $X \times V \subseteq X \times \mathbf{P}^{3}$, and hence by $7.1 H$ projects onto $X$. Let $D$ be the projection of $H$ to $V ; D$ is a cofinite subset of $V$. Then $H$ is the graph of a bijective morphism between $X$ and $D$.

This ends the proof of Theorem A.

Recall now the situation of Theorem $\mathrm{B}^{\prime} . \quad D$ is an ample Zariski geometry; we use the full language $L_{\text {full }}$, including constants for the elements of $D$. We took an elementary extension $D^{*}$ of $D$, with respect to the language $L_{\text {full }}$. When we wish to refer to the original $D$ within $D^{*}$, we will call it $M$; it is an elementary submodel of $D^{*}$. We let $G$ be the group of automorphisms of the triple $\left(D^{*}, D, L_{\text {full }}\right)$; we do not require that they fix pointwise either $D$ or $L_{\text {full }}$. Any automorphism of $D$ as a Zariski geometry extends to an element of $G$. The natural language $L_{\text {nat }}$ is the set of relations of $L_{\text {full }}$ left fixed by $G$.

In the proof of Theorem $\mathrm{B}^{\prime}$, the words definable, 0-definable, etc. will be with respect to $L_{\text {full }}$, except where explicitly indicated to the contrary.

Theorem $\mathbf{B}^{\prime}$. Let $D$ be an ample Zariski geometry, and let $L_{\text {nat }}$ be the natural language of $D$. There exists a field $K$, a smooth curve $C$ over $K$, and a surjective finite-to-one Zariski map $f: D \rightarrow C$, all 0-definable in $L_{\text {nat }}$.

Proof. We observe that as an $L_{\text {full-structure, }} D$ is strongly minimal, and hence it is also strongly minimal as an $L_{\text {nat }}$-structure.

Since $L_{\text {full }}$ contains names for all elements of a model $M$, by Theorem B there exists an algebraically closed field $K$ interpretable in $L_{\text {full }}$ without parameters, and a surjective, finite-to-one definable map $D \rightarrow \mathbf{P}^{1}(K)$. Let $P$ be the complete type $D^{*}-M$. By 9.2 there exists a definable map $f^{*}: P \rightarrow C^{*}$ such that $C^{*}$ is $K$ internal, and $f: P \rightarrow C^{*}$ is $K$-external. $f^{*}$ may be extended to a definable map $f: D \rightarrow C$, where $C$ is a $K$-internal definable set. Observe that $P$ is $G$-invariant.

Let $E=E(f, K)=\left\{(x, y) \in D^{2}: f(x)=f(y)\right\}$, let $E^{c}$ be the Zariski closure of $E$, and let $E \#$ be the union of one-dimensional components of $E^{c}$ (the other components are finite). 
Claim. E\# is $L_{\text {nat-definable. }}$

Indeed any automorphism of $D$ must carry $E$ to $E^{\prime}=E\left(f^{\prime}, K^{\prime}\right)$, where $K^{\prime}$ is another definable algebraically closed field, $C^{\prime}$ is $K^{\prime}$-internal, and the restriction of $f^{\prime}$ to $P$ is $K^{\prime}$-external. By [Po], and using $7.10, K, K^{\prime}$ are isomorphic by a 0 -definable isomorphism in $L$. Hence $K$-internal is the same as $K^{\prime}$-internal, and $K$ external as $K^{\prime}$-external. By $9.3, f^{\prime} \mid P=h f^{*}$ for some $h$, and $f^{*}=h^{\prime} f^{\prime} \mid P$ for some $h^{\prime}$. Hence $E(f, K)$ and $E\left(f^{\prime}, K^{\prime}\right)$ coincide on $P$. Now $E, E^{\prime}$ are one-dimensional, and so $E, E^{\prime}$ differ by at most a finite set, and hence $E \#=E^{\prime} \#$. Thus $E \#$ is $G$-invariant, so it is definable in $L_{\text {nat }}$.

As $E \#$ is purely one-dimensional, and differs from an equivalence relation on $D$ by a finite set, one can show that $E \#$ is an equivalence relation. Clearly it is symmetric and reflexive. If $\left(a^{\prime}, b^{\prime}\right)$ and $\left(a^{\prime}, c^{\prime}\right)$ are in $E \#$, let $a$ be a generic element of $D$, and let $a b \rightarrow a^{\prime} b^{\prime}, a c \rightarrow a^{\prime} c^{\prime}$ be specializations, with $(a, b) \in E \#$ and $(a, c) \in E \#$. By 5.14 we can choose $b, c$ such that $a b c \rightarrow a^{\prime} b^{\prime} c^{\prime}$. We have $(b, c) \in E$ and hence $(b, c) \in E \#$, so also $\left(b^{\prime}, c^{\prime}\right) \in E \#$. (We are using here the fact that $a \rightarrow a^{\prime}$ is regular, and $\operatorname{dim}(D)=1$.)

As in the proof of Theorem A, or of 1.1, $C$ may be given the structure of a curve over $K$; we may take $C$ to be smooth and complete. We have a definable map $f^{*}: P \rightarrow C$; let $F$ be the Zariski closure of $f^{*}$ in $P \times C$. Using 4.8 we see that $F$ is the graph of a partial function from $D$ to $C$. By 7.4, $F$ is total. We write $F^{*} R$ for the inverse image of $R \subseteq C^{n}$ under the map $(F, \ldots, F)$.

Let $H$ be the group of automorphisms of $C$ as a curve over $K$. Then $H$ is a definable group, with connected component $H^{\prime} . H^{\prime}$ is sharply transitive on $C^{k}$ for some $k=0,1,2$ or 3 . $H$ acts on $C^{n}$ for every $n$. Let $L(C)$ be the set of definable relations on $C$ that are invariant under the action of $H$. It is easy to see that every definable relation on $C$ is $L(C)$-definable with parameters (see [Hr6], Proposition $5)$. Indeed in the present case, for every definable relation $R$ on $C^{m}$ there exists an $H$-invariant relation $R^{\prime}$ on $C^{m+k}$ such that $R=R^{\prime}(b)$ for some $b \in C^{k}$.

Claim. Let $R$ be a closed irreducible subset of $C^{n}$ (defined over the prime field). Then $F^{*} R$ is $L_{\text {nat }}$-definable.

Proof. By the remark preceding the claim, it suffices to prove this when $R$ is in $L(C)$. Let $g$ be an element of $G$; we must show that $g\left(F^{*} R\right)=F^{*} R . g$ takes $(C, K, R, F)$ to $\left(C^{\prime}, F^{\prime}, R^{\prime}, K^{\prime}\right)$, and $g\left(F^{*} R\right)=F^{* *} R^{\prime}$. Composing $F^{\prime} F^{-1}$ (viewed as bijections from $D / E \#$ to a subset of $C$ ) and taking Zariski closure we get a definable bijection $H: C \rightarrow C^{\prime}$. It is a morphism, and hence a Zariski isomorphism. Hence $g^{-1} H$ is also a Zariski isomorphism from $C$ to $C$. By 1.1 it can be obtained by composing a field automorphism with an element of $H$. But $R$ is in $L(C)$ and is defined over the prime field, so $R$ is invariant under $H$ and under automorphisms of the field. Thus $g^{-1} H R=R$, and it follows that $g\left(F^{*} R\right)=F^{* *} R^{\prime}$.

It follows that $C$ and the structure on it can be found in $L_{\text {nat }}^{\mathrm{eq}}$, proving the theorem.

Example 9.10. It is not true that every closed subset of $D^{n}$ is definable in $L_{\text {nat }}$, with parameters from $D$. For instance one may take a double cover $D=(Z / 2 Z) \times$ $K$, where $K$ is an uncountable algebraically closed field. To describe the structure on $D$, let:

$L_{0}=\left\{+, \cdot, 0,1 ; \pi_{2}\right\}$ (the language of $K$ and the projection to $K$ ).

$L_{1}=L_{0} \cup\left\{\pi_{1}\right\}$ (add the projection to $\{0,1\}$, or equivalently a predicate for

$\{0\} \times K$ and for $\{1\} \times K)$.

$L_{2}=L_{1} \cup\left\{c_{i}\right\}_{i}$ (add constants for all elements of $K$ ). 
Define $g_{i}: D \rightarrow D$ (in $\left.L_{2}\right)$ by: $g_{i}(\iota, y)=\left(\iota, y+c_{i}\right)$ and let:

$$
L=L_{0} \cup\left\{g_{i}\right\}_{i} \text {. }
$$

Now we consider $D$ in the language $L$ as a Zariski geometry. Note that $L \subseteq L_{2}$, and so $L_{\text {nat }} \subseteq\left(L_{2}\right)_{\text {nat }}$. But adding constants does not change the Zariski structure, and hence not the natural language, so $\left(L_{2}\right)_{\text {nat }}=\left(L_{1}\right)_{\text {nat }}$. Thus $L_{\text {nat }}$ is a countable language. Hence $L_{\text {nat }}$ is a sublanguage of $L_{0} \cup\left\{g_{i}: i \in I\right\}$, where $I$ is countable. Choose $c_{j}$ outside the $Q$-space generated by the $\left\{g_{i}: i \in I\right\}$. Then one sees easily by an automorphism argument that $g_{j}$ is not definable with parameters in $L_{0} \cup\left\{g_{i}: i \in I\right\}$. This shows that $L_{\text {nat }}+$ parameters $\neq L$.

Example 9.11. If we took the natural language of a higher-dimensional manifold $M$, it would no longer be true that a 0 -definable field can be found.

Let $K$ be an algebraically closed field of positive characteristic, $n>1$, and let $\phi(y)$ be the Frobenius map taken to some negative power above $-n$. Define a permutation $\sigma$ on $K^{n}$ by mapping $\left(x_{1}, \ldots, x_{n}\right)$ to $\left(\phi x_{n}, x_{1}, x_{2}, \ldots, x_{n-1}\right)$. We will choose $D$ as a reduct of $K^{n}$, taking only the closed subsets of $D^{l}$ left-invariant under $\sigma$. Note that $\sigma$ is definable in $K$ and $\sigma^{n}$ is an automorphism of $K^{n}$, so that if $C$ is a 0-closed set in $\left(K^{n}\right)^{m}$, then $C \cup \sigma C \cup \cdots \cup \sigma^{n-1} C$ is 0-definable in $D^{m}$. But here there is no 0-definable field.

It can be shown that an $\aleph_{1}$-categorical structure interpreting a pure infinite field must interpret such a field over $\operatorname{acl}(\emptyset)$. The above example shows the algebraic parameters cannot be removed, even in the almost strongly minimal context.

\section{Nonalgebraic Zariski geometries}

By Theorem B, a new ample Zariski geometry can only be obtained as a finite cover of the projective line over an algebraically closed field. We describe a method for obtaining such covers.

We consider abstract groups $G$ acting on Zariski geometries $X$ so that the graph of each element of $g$ is closed irreducible, i.e., $g$ is a morphism. We assume that each orbit of $G$ on $X$ is regular or degenerate; in other words if $g x=x$, then $g^{\prime} x=x$ for all $g^{\prime}$ or $g=1$. Such actions will be called semi-free.

Proposition 10.1. Let $X$ be a Zariski structure, $G$ an abstract group, and let there be given a semi-free action of $G$ on $X$. Let $i: G^{*} \rightarrow G$ be a group homomorphism with finite kernel $H$. Then there exist a Zariski geometry $X^{*}$, a semi-free action of $G^{*}$ on $X^{*}$, and a closed, surjective Zariski map $j: X^{*} \rightarrow X$, such that $i, j$ are compatible. If $X$ is complete, then $X^{*}$ is complete.

Proof. Let $X_{0}=\{x \in X: g x=x$ for all $g \in G\}$. By the semi-freeness assumption, for any $g \neq 1, X_{0}$ is the set of fixed points of $g$; hence $X_{0}$ is a closed subset of $X$. If $X_{0}=X$ we may take $X^{*}=X$, and $G^{*}$ acting trivially on $X^{*}$; so we may assume $X_{0}$ is finite.

Let $X^{*}$ be a set on which there is a semi-free action of $G^{*}$, such that $G^{*}$ acts trivially on a set $X_{0}^{*}$ of the same size as $X_{0}$, and regularly on each orbit outside $X_{0}^{*}$; and such that the number of nondegenerate $G^{*}$-orbits equals the number of nondegenerate $G$-orbits on $X$. Define an equivalence relation $E$ on $X^{*}: x E y$ iff there exists $h \in H$ with $h x=y$. Then $E$ is respected by the action of $G^{*}$; so $G^{*}$ acts on the quotient $X^{*} / E$, in such a way that $H$ acts trivially. Thus $X^{*} / E$ can be considered as a $G$-set. It has the same number of degenerate orbits as does $X$, 
and the same number of regular ones, hence is isomorphic to $X$ as a $G$-set. Thus there exists a map $j: X^{*} \rightarrow X$ carrying $X_{0}^{*}$ to $X_{0}$, and such that $j g^{*}=i\left(g^{*}\right) j$.

If $U$ is a closed subset of $X^{n}$, and $w$ a partial map from $n^{2}$ into $G^{*}$, define

$$
\begin{gathered}
F(U, w)=\left\{\left(x_{1}, \ldots, x_{n}\right) \in X^{* n}: w\left(\nu, \nu^{\prime}\right)\left(x_{\nu}\right)=x_{\nu^{\prime}} \text { for }\left(\nu, \nu^{\prime}\right) \in \operatorname{dom}(w),\right. \\
\text { and } \left.\left(j x_{1}, \ldots, j x_{n}\right) \in U\right\} .
\end{gathered}
$$

We call the pair $(U, w)$ normal if the following conditions hold:

(i) $\operatorname{dom}(w)$ is an equivalence relation. $w(\nu, \nu)=1$.

(ii) If $\left(\nu, \nu^{\prime}\right) \in \operatorname{dom}(w)$, then $U \subseteq\left\{\left(x_{1}, \ldots, x_{n}\right): i\left(w\left(\nu, \nu^{\prime}\right)\right)\left(x_{\nu}\right)=x_{\nu^{\prime}}\right\}$.

(iii) If $\left(\nu, \nu^{\prime}\right),\left(\nu^{\prime}, \nu^{\prime \prime}\right) \in \operatorname{dom}(w)$, then $w\left(\nu, \nu^{\prime \prime}\right)=w\left(\nu^{\prime}, \nu^{\prime \prime}\right) w\left(\nu, \nu^{\prime}\right)$.

(iv) Let $J(U)=\left\{\nu: U \subseteq\left\{x: x_{\nu} \in X_{0}\right\}\right\}$. Then $w\left(\nu, \nu^{\prime}\right)=1$ whenever $\nu, \nu^{\prime} \in J$ and $\left(\nu, \nu^{\prime}\right) \in \operatorname{dom}(w)$.

Claim 1. If $(U, w)$ is normal, then any point of $U$ lifts to a point of $F(U, w)$.

Proof. Let $\left(x_{1}, \ldots, x_{n}\right) \in U$. Let $R$ be a set of representatives for the equivalence relation $\operatorname{dom}(w)$. Choose $x_{\nu}^{*} \in X^{*}$ with $j\left(x_{\nu}^{*}\right)=x_{\nu}(\nu \in R)$. For arbitrary $\mu \leq n$, let $\nu \in R$ be such that $(\nu, \mu) \in \operatorname{dom}(w)$, and let $x_{\mu}^{*}=w(\nu, \mu)\left(x_{\nu}\right)$. Then $j\left(x_{\mu}^{*}\right)=i(w(\nu, \mu))\left(x_{\nu}\right)=x_{\mu} ;$ and $\left(x_{1}^{*}, \ldots, x_{n}^{*}\right) \in F(U, w)$.

Claim 2. For any $U, w$ there exists a normal $U^{\prime}, w^{\prime}$ with $F(U, w)=F\left(U^{\prime}, w^{\prime}\right)$.

Proof. Let $J=\left\{\nu \in\{1, \ldots, n\}\right.$ : for all $\left.x \in F(U, w), x_{\nu} \in X_{0}^{*}\right\}$. Without changing $F(U, w)$, we may replace $U$ by $U \cap\left\{x \in X^{n}: x_{\nu} \in X_{0}\right.$ for $\left.\nu \in J\right\}$. Further we may change $w$ so that $w\left(\nu, \nu^{\prime}\right)=1$ when $\left(\nu, \nu^{\prime}\right) \in \operatorname{dom}(w)$ and $\nu, \nu^{\prime} \in J$. (i) may be met by adding $\left(\nu, \nu^{\prime \prime}\right)$ to $\operatorname{dom}(w)$ whenever it is missing and $\left(\nu, \nu^{\prime}\right)$ and $\left(\nu^{\prime}, \nu^{\prime \prime}\right) \in \operatorname{dom}(w)$, and letting $w\left(\nu, \nu^{\prime \prime}\right)=w\left(\nu^{\prime}, \nu^{\prime \prime}\right) w\left(\nu, \nu^{\prime}\right)$; repeating this process will make $\operatorname{dom}(w)$ an equivalence relation without changing $F(U, w)$. If (iii) fails, then

$$
w\left(\nu, \nu^{\prime \prime}\right)^{-1} w\left(\nu^{\prime}, \nu^{\prime \prime}\right) w\left(\nu, \nu^{\prime}\right) \neq 1,
$$

yet $w\left(\nu, \nu^{\prime \prime}\right)^{-1} w\left(\nu^{\prime}, \nu^{\prime \prime}\right) w\left(\nu, \nu^{\prime}\right) x_{\nu}=x_{\nu}$ whenever $x \in F(U, w)$, so $\nu \in J$ and similarly $\nu^{\prime}, \nu^{\prime \prime} \in J$; but then $w\left(\nu, \nu^{\prime \prime}\right)=w\left(\nu^{\prime}, \nu^{\prime \prime}\right)=w\left(\nu, \nu^{\prime}\right)=1$, a contradiction. Finally (ii) may be met by reducing $U$.

Claim 3. There is no infinite descending chain of sets $F(U, w)$.

Proof. Suppose such a chain $F\left(U_{i}, w_{i}\right)$ exists. We may assume each $\left(U_{i}, w_{i}\right)$ is normal. Let $U_{i}^{*}$ be the intersection of all $U_{j}, j \leq i$. Then $F\left(U_{i}^{*}, w_{i}\right)=F\left(U_{i}, w_{i}\right)$. By the descending chain condition on closed subsets of $X^{n}, U_{i}^{*}=U$ for all large enough $i$; so we may assume all $U_{i}=U$. Let $J=\{\nu \in\{1, \ldots, n\}$ : for all large enough $i$ and all $\left.x \in F\left(U_{i}, w_{i}\right), x_{\nu} \in X_{0}\right\}$, and let $w_{i}^{\prime}$ be $w_{i}$ restricted to the complement of $J$. Then $F\left(U, w_{i}^{\prime}\right)=F\left(U, w_{i}\right)$ for large $i$, so we may assume $\operatorname{dom}\left(w_{i}\right)$ does not meet $J \times\{1, \ldots, n\}$. Now suppose $\left(\nu, \nu^{\prime}\right) \in \operatorname{dom}\left(w_{i}\right) \cap \operatorname{dom}\left(w_{j}\right)$ and $w_{i}\left(\nu, \nu^{\prime}\right) \neq w_{j}\left(\nu, \nu^{\prime}\right)$. Then for any $k \geq i, j$ and any $x=\left(x_{1}, \ldots, x_{n}\right) \in F\left(U_{k}, w_{k}\right)$, we have $x \in F\left(U_{i}, w_{i}\right)$ and $x \in F\left(U_{j}, w_{j}\right)$, so $w_{i}\left(\nu, \nu^{\prime}\right) x_{\nu}=x_{\nu^{\prime}}=w_{j}\left(\nu, \nu^{\prime}\right) x_{\nu}$, hence $x_{\nu} \in X_{0}$. Thus $\nu \in J$, so $w_{i}\left(\nu, \nu^{\prime}\right)=1=w_{j}\left(\nu, \nu^{\prime}\right)$, a contradiction. Thus $w_{i} \cup w_{j}$ is a function for all $i, j$; so we may assume $w_{i} \subseteq w_{j}$ when $i \leq j$. But then the value of $w_{i}$ stabilizes for large $i$, so the sets $F\left(U, w_{i}\right)$ are eventually equal; a contradiction.

Claim 4. The sets $F(U, w)$ are closed under intersections.

Proof. By the previous claim, it suffices to consider the intersection of two sets $F(U, w)$ and $F\left(U^{\prime}, w^{\prime}\right)$. Let $Q=\left\{(i, j) \in \operatorname{dom}(w) \cap \operatorname{dom}\left(w^{\prime}\right): w(i, j) \neq w^{\prime}(i, j)\right\}$, and let $Q_{1}=\{i:(i, j) \in Q$ for some $j\}$. Let $w^{\prime \prime}$ be the function with domain equal to $\operatorname{dom}(w) \cup \operatorname{dom}\left(w^{\prime}\right)-Q$, and let $U^{\prime \prime}=U \cap U^{\prime} \cap\left\{\left(x_{1}, \ldots, x_{n}\right): x_{i} \in X_{0}\right.$ for 
$\left.i \in Q_{1}\right\}$. Then

$$
F(U, w) \cap F\left(U^{\prime}, w^{\prime}\right)=F\left(U^{\prime \prime}, w^{\prime \prime}\right) .
$$

We define a closed subset of $X^{* n}$ to be a finite union of sets of the form $F(U, w)$ and singletons. It follows from the previous two claims that the closed sets form a Noetherian topology.

Claim 5. Let $(U, w)$ be normal, $U$ infinite. Then $F(U, w)$ is irreducible iff:

(i) $U$ is irreducible.

(ii) If $U \subseteq\left\{\left(x_{1}, \ldots, x_{n}\right): f(\nu)=x_{\nu^{\prime}}\right\}, f \in G$, then $\left(\nu, \nu^{\prime}\right) \in \operatorname{dom}(w)$.

If these conditions hold, then $\operatorname{dim} F(U, w)=\operatorname{dim} U$.

Proof. If $F(U, w)$ is irreducible, then using Claim 1 one sees easily that $U$ is irreducible. If $U \subseteq\left\{\left(x_{1}, \ldots, x_{n}\right): f\left(x_{\nu}\right)=x_{\nu^{\prime}}\right\}$, let $C=\left\{w^{\prime}: w \subseteq w^{\prime}, \operatorname{dom}\left(w^{\prime}\right)=\right.$ $\operatorname{dom}(w) \cup\left\{\left(\nu, \nu^{\prime}\right)\right\}$, and $\left.i w^{\prime}\left(\nu, \nu^{\prime}\right)=f\right\}$. Then $C$ is finite (of the same size as $H$ ), and $F(U, w)=\bigcup\left\{F\left(U, w^{\prime}\right): w^{\prime} \in C\right\}$. By irreducibility, $F(U, w)=F\left(U, w^{\prime}\right)$ for some $w^{\prime}$. The proof of Claim 1 now shows that $w=w^{\prime}$. This proves (ii).

Now suppose (i), (ii) hold, but $F(U, w)$ is contained in a finite union of proper subsets $F\left(U_{i}, w_{i}\right)$ and singletons; we may take $\left(U_{i}, w_{i}\right)$ normal. Using Claim 1, we see that $U$ is contained in the union of the sets $U_{i}$ and singletons. Since $U$ is irreducible and infinite, it must be contained in some $U_{i}$. Since $\left(U_{i}, w_{i}\right)$ is normal, $U \subseteq U_{i} \subseteq\left\{\left(x_{1}, \ldots, x_{n}\right): i\left(w_{i}\left(\nu, \nu^{\prime}\right)\right)\left(x_{\nu}\right)=x_{\nu^{\prime}}\right\}$ whenever $\left(\nu, \nu^{\prime}\right) \in \operatorname{dom}\left(w_{i}\right)$. By (ii) it follows that $\operatorname{dom}\left(w_{i}\right) \subseteq \operatorname{dom}(w)$. One concludes easily that $w_{i}=w$ and $F\left(U_{i}, w_{i}\right)=F(U, w)$.

Claim 6. Let $(U, w)$ be normal, and let $V$ be a closed irreducible subset of $U$. Then there exists $w^{\prime}$ such that $\left(V, w^{\prime}\right)$ is normal and $F\left(V, w^{\prime}\right)$ is irreducible, and contained in $F(U, w)$. In fact $F(V, w)$ is the union of finitely many such sets.

Proof. Let $e$ be the set of pairs $\left(\nu, \nu^{\prime}\right)$ such that $V \subseteq\left\{\left(x_{1}, \ldots, x_{n}\right): f\left(x_{\nu}\right)=x_{\nu^{\prime}}\right\}$ for some $f \in G$. Then $e$ is an equivalence relation, refining $\operatorname{dom}(w)$. All the maps $w^{\prime}$ we will obtain will have domain $e$.

Let $v^{*}=\left(x_{1}, \ldots, x_{n}\right)$ be any element of $F(V, w)$. Define $w^{\prime}=w^{\prime}\left(v^{*}\right)$ as follows. Let $\left(\nu, \nu^{\prime}\right) \in e$. If $x_{\nu}=x_{\nu^{\prime}} \in X_{0}^{*}$, let $w^{\prime}\left(\nu, \nu^{\prime}\right)=1$. Otherwise let $w^{\prime}\left(\nu, \nu^{\prime}\right)$ be the unique $g \in G^{*}$ such that $g x_{\nu}=x_{\nu^{\prime}}$. One verifies immediately that $\left(V, w^{\prime}\right)$ is normal, and $v^{*} \in F\left(V, w^{\prime}\right)$. The conditions of Claim 5 are also satisfied. If $\left(\nu, \nu^{\prime}\right) \in \operatorname{dom}(w)$, then by normality of $(U, w)$ we have $\left(\nu, \nu^{\prime}\right) \in e$; and since $v^{*} \in F(V, w)$, either $\nu, \nu^{\prime} \in J(V)$ or $w^{\prime}\left(\nu, \nu^{\prime}\right)=w\left(\nu, \nu^{\prime}\right)$. Thus $v^{*} \in F\left(V, w^{\prime}\right) \subseteq F(V, w)$. Since there are only finitely many possibilities for $w^{\prime}$, the claim is proved.

Claim 7. Let $(U, w)$ be normal, $U$ irreducible. Every component of $F(U, w)$ projects onto $U$, and has dimension equal to $\operatorname{dim}(U)$.

Proof. Applying Claim 6 to $U$, we see that $F(U, w)$ is covered by irreducible sets $F\left(U, w^{\prime}\right)$, where $F\left(U, w^{\prime}\right)$ projects onto $U$. Every component of $F(U, w)$ must be one of these sets. The fact that $\operatorname{dim} F\left(U, w^{\prime}\right) \geq \operatorname{dim}(U)$ also follows from Claim 6 , using induction on $\operatorname{dim}(U)$. For the other inequality, any infinite closed irreducible subset of $F\left(U, w^{\prime}\right)$ has the form $F\left(V, w^{\prime \prime}\right)$ for some closed irreducible $V \subseteq U$ and some $w^{\prime \prime}$ satisfying the conditions in Claim 5. It then follows from (ii) of the definition of normality, and (ii) of Claim 5, that if $U=V$, then $\operatorname{dom}\left(w^{\prime}\right)=\operatorname{dom}\left(w^{\prime \prime}\right)$; and hence using (iv) of the definition of normality, that $w^{\prime}=w^{\prime \prime}$. Thus if $F\left(V, w^{\prime \prime}\right)$ is a proper subset of $F\left(U, w^{\prime}\right)$, then $V \neq U$, and by induction on dimension we may conclude $\operatorname{dim} F\left(U, w^{\prime}\right) \leq \operatorname{dim}(U)$.

The verification of property $(\mathrm{Z} 0)$ of the definition of a Zariski geometry is trivial. We proceed to show the other conditions hold. 
(Z1) Let $C=F(U, w) \subseteq X^{* n}$ be irreducible, and let $\pi$ be the projection from $X^{* n}$ to $X^{* k}$. We must show that for some proper closed subset $F^{*}$ of $\operatorname{cl}(\pi C), \pi C \supseteq$ $\operatorname{cl}(\pi C)-F^{*}$. We may assume $(U, w)$ is normal.

We first make the following observation. Let $\pi^{\prime}$ be some projection to $\prod_{i \in s} X^{*}$, where $s$ is a subset of the coordinates; and assume that for any $\nu \notin s$, there is some $\nu^{\prime} \in s$ with $\left(\nu^{\prime}, \nu\right) \in \operatorname{dom}(w)$. Then $\pi^{\prime} C$ is closed. Indeed choose, for any $\nu \notin s$, some $\nu^{\prime} \in s$ with $\left(\nu^{\prime}, \nu\right) \in \operatorname{dom}(w)$, and let $h(\nu)$ be the morphism $i\left(w\left(\nu^{\prime}, \nu\right)\right)$. Let $H$ be the map carrying $\left(x_{\nu}: \nu \in s\right) \in \prod_{i \in s} X$ to

$$
\left(x_{\nu}: \nu \in s\right) \wedge\left(h(\nu)\left(x_{\nu^{\prime}}\right): \nu^{\prime} \notin s\right) \in \prod_{i \in s} X \times \prod_{i \notin s} X=X^{n} .
$$

Then $H$ is a morphism, so $U^{\prime}=H^{-1}(U)$ is closed. But $\pi^{\prime} C=F\left(U^{\prime}, w^{\prime}\right)$, where $w^{\prime}$ is the restriction of $w$ to $s$. Thus $\pi^{\prime} C$ is closed.

By virtue of this observation, factoring $\pi$ into two projections, we may assume: there are no $\left(\nu^{\prime}, \nu\right) \in \operatorname{dom}(w)$ with $\nu^{\prime} \leq k, \nu>k$. Let $\pi$ also denote the projection from $X^{n}$ to $X^{k}$, and let $V$ be a proper closed subset of $\operatorname{cl}(\pi U)$ such that $\pi U \supseteq \operatorname{cl}(\pi U)-V$. Let $w^{\prime}$ denote the restriction of $w$ to $\{1, \ldots, k\}$. Then $\pi C \supseteq F\left(\operatorname{cl}(\pi U), w^{\prime}\right)-F\left(V, w^{\prime}\right)$. Using Claim 1 we see that $F\left(V, w^{\prime}\right)$ is a proper subset of $F\left(\operatorname{cl}(\pi U), w^{\prime}\right)$, so (Z1) holds. Moreover, if $V=\emptyset$, then $F\left(V, w^{\prime}\right)=\emptyset$, so if $X$ is complete, then so is $X^{*}$.

(Z2) It suffices to show this for the sets $C=F(U, w) \subseteq X^{n+1}$. If $(\nu, n+1) \in$ $\operatorname{dom}(w)$ for some $\nu \leq n$, then clearly $|C(a)| \leq 1$ for all $a \in X^{n}$. Otherwise $C(a)=\{b \in X:(j a, j b) \in U\}$. So either $C(a)=X^{*}$ or $|C(a)| \leq|U(j a)| \cdot|H|$.

(Z3) Let $(U, w)$ be normal, $F(U, w)$ irreducible, and consider the intersection with the diagonal $\Delta^{*}=\left\{x^{*}: x_{1}^{*}=x_{2}^{*}\right\}$. We must show that $F(U, w) \cap \Delta^{*}$ is the union of irreducible closed sets of dimension $\geq \operatorname{dim}(U)-1$.

Let $\Delta=\left\{x \in X^{n}: x_{1}=x_{2}\right\}$, and let $U^{\prime}=U \cap \Delta$. By the dimension theorem for $X, U^{\prime}$ is the union of irreducible components $U_{i}$ of dimension at least $\operatorname{dim}(U)-1$. By Claim $6, F\left(U_{i}, w\right)$ is the union of irreducible closed sets $F\left(U_{i}, w_{i j}\right)$, with $\left(U_{i}, w_{i j}\right)$ normal, and by Claim 7 they all have dimension $\geq \operatorname{dim}(U)-1$. Now by (ii) of Claim 5, and since each $U_{i} \subseteq\left\{x: x_{1}=x_{2}\right\},(1,2) \in \operatorname{dom}\left(w_{i j}\right)$ for all $i, j$. If $w_{i j}(1,2)=1$, then $F\left(U_{i}, w_{i j}\right)$ is contained in $F(U, w) \cap \Delta^{*}$. If $v^{*} \in F(U, w) \cap \Delta^{*}$, let $v=j v^{*}$, and let $i$ be such that $v \in U_{i}$; then defining $w_{i j}=w^{\prime}\left(v^{*}\right)$ as in the proof of Claim 6, we see that $v^{*} \in F\left(U_{i}, w_{i j}\right)$ for some $(i, j)$ such that $w_{i j}(1,2)=1$. Thus $F(U, w) \cap \Delta^{*}$ is the union of all the $F\left(U_{i}, w_{i j}\right)$ with $w_{i j}(1,2)=1$. This finishes the proof.

Remark 10.2. An alternative proof of 10.1 is possible along the following lines. Define $X^{*}$ as above. Consider $X^{*}$ as a structure whose basic relations are the elements of $G^{*}$ (considered as binary relations on $X^{*}$ ) and the pullbacks via $j$ of the closed subsets of $X^{n}$. The subsets defined by positive atomic formulas (with parameters) are called closed. One shows:

(*) Let $M, N$ be models of the theory $T$ of $X^{*}, A$ a substructure of $M$, and $f: A \rightarrow N$ a homomorphism. Then $f$ extends to a homomorphism from $M$ into some elementary extension of $N$. If $f$ is $1-1, f$ extends to an isomorphism between elementary extensions of $M$ and of $N$.

From this it follows, by standard model-theoretic arguments, that $T$ admits quantifier elimination; and further that collection of closed sets is closed under projections. In the proof of $(*)$ one notes: 
(**) Suppose $M, N, A$ are as in (*), and $f$ is compatible with an isomorphism $f_{X}: X^{M} \rightarrow X^{N}$. Then $f$ extends to an isomorphism of $M$ and $N$, also compatible with $f_{X}$.

This shows that $X^{*}$ has Morley rank 1, and induces no new structure on $X$. Finally, to prove the dimension theorem amounts to verifying:

$(* * *)$ Let $h: A \rightarrow C$ be a homomorphism between finitely generated substructures of models of $T$. Suppose $a_{1}, a_{2} \in A$ and $h a_{1}=h a_{2}$. Then there exists a substructure $B$ of a model of $T$, and homomorphisms $h^{\prime}: A \rightarrow B, h^{\prime \prime}: B \rightarrow C$, such that $h=h^{\prime \prime} h^{\prime}$, and $\operatorname{rk}(B) \geq \operatorname{rk}(A)-1$.

Proof of Theorem C. Proposition 10.1 makes a variety of constructions possible, of which we give one example. Let $k$ be an algebraically closed field of characteristic 0 . If $C$ is a nonsingular curve over $K$, and $f: C-F_{0} \rightarrow C-F_{1}$ is a definable bijection between two cofinite subsets of $C$, then $f$ agrees (up to a finite number of points) with a bijective morphism $f^{*}: C \rightarrow C$, an algebraic automorphism of $C$. $f^{*}$ is uniquely determined by $f$. This gives a map from the group of definable bijections of $C$ to the group $\operatorname{Aut}_{K}(C)$ of automorphisms of $C$ over $K$; the kernel is the group of maps with finite support.

Let $X$ be an elliptic curve with transcendental $j$-invariant, with a fixed zero point $p$ on $X$. Choose two independent generic elements $a, b$ of $X$; let $t_{a}, t_{b}$ denote the translation maps by $a, b$. Let $G$ be the group of automorphisms of $X$ generated by $t_{a}, t_{b}$. So $G$ is isomorphic to $Z^{2}$. Let $G^{*}$ be the group generated formally by elements $T_{a}, T_{b}$ with the relations: $\left[T_{a}, T_{b}\right]^{2}=\left[T_{a},\left[T_{a}, T_{b}\right]\right]=\left[T_{b},\left[T_{a}, T_{b}\right]\right]=1$. Let $H$ be the subgroup generated by the commutator $\left[T_{a}, T_{b}\right]$. Then $H$ is the 2-element group, and we have a group homomorphism $i: G^{*} \rightarrow G$ with kernel $H$, taking $T_{a}$ to $t_{a}, T_{b}$ to $t_{b}$. Let $X^{*}$ be the Zariski geometry given by 10.1 for this data. We claim that $X^{*}$ is not interpretable in any algebraically closed field.

Suppose $X^{*}$ is so interpretable, over some algebraically closed field $K$. Observe that $k$ is interpretable in $X$, hence in $X^{*}$, and so also in $K$. By [Po], since $k$ is interpretable in $K$, it is in $K$ definably isomorphic to $K$. Thus $X$ can be viewed as an elliptic curve over $K$, and $j: X^{*} \rightarrow X$ is a definable 2-1 map from some definable set into $X$.

$X^{*}$ can be viewed as the union of (possibly incomplete) curves $C_{1}, \ldots, C_{m}$ over $K$, perhaps with a finite number of additional points added. If $x$ is a generic point of $X$, then $j^{-1}(x)$ must have a point from each $C_{i}$. Since the map $j: X^{*} \rightarrow X$ is $2-1$, we have $m \leq 2$. If $m=2$, then for generic $x, j^{-1}(x)$ has one point from $C_{1}$ and one from $C_{2}$; since the element $\left[T_{a}, T_{b}\right]$ of $G^{*}$ permutes $j^{-1}(x)$, it carries $C_{1}$ to $C_{2}$ (perhaps with finitely many points excepted). However the maps $T_{a}$ and $T_{b}$ must either preserve $C_{1}, C_{2}$ or permute them (up to a finite number of points); in any of the four possibilities, the commutator $\left[T_{a}, T_{b}\right]$ leaves each of the two component curves invariant. This contradiction shows that $m=1$.

Thus $X^{*}$ can be identified with a single curve (up to a finite number of points); we may take this curve $C$ to be complete and nonsingular. By the remark on nonsingular curves in the first paragraph, the action of $G^{*}$ on $X^{*}$ induces an action of $G$ on $C$ by algebraic automorphisms. Since no non-identity element of $G^{*}$ fixes a cofinite subset of $X^{*}$, this gives an embedding of $G^{*}$ into $\operatorname{Aut}_{K}(C)$. In particular, Aut $_{K}(C)$ is infinite. By [Ha], p. 305, it follows that $C$ has genus $\leq 1$. We also have a rational map from $C$ to $X$, so $X$ has genus $\geq 1$. Thus $C$ has genus 1 . It is isogeneous to $X$, hence also has a transcendental $j$-invariant. 
By [Ha], p. 321, the subgroup $H$ of $\operatorname{Aut}_{K}(C)$ fixing $p$ has two elements, the identity and the group inverse. The group $\operatorname{Aut}_{K}(C)$ has the structure of a semidirect product of $W$ with an Abelian group $A$ (the group of translations of $C$ ), with $W$ acting on $A$ by inversion. It follows that any two elements of $\operatorname{Aut}_{K}(C)$ not of order 2 lie inside $A$, and hence commute. In $G^{*}$ however, $T_{a}, T_{b}$ do not have order 2 , and do not commute. This contradicts the observation that $G^{*}$ embeds into Aut $(C)$, and shows that $X^{*}$ is not interpretable in an algebraically closed field.

\section{REFERENCES}

[AE] E. Artin, Geometric algebra, Interscience, New York, 1957; Springer-Verlag, Berlin and New York, 1988. MR 90h:51003

[AM] M. Artin, Algebraic spaces, Yale University, 1969. MR 53:10795

[B] E. Bouscaren, Hrushovski's group configuration, The Model Theory of Groups (A. Nesin and A. Pillay, eds.), Notre Dame Math. Lectures, no. 11, Univ. Notre Dame Press, Notre Dame, IN, 1989. CMP 21:09

[BL] T. J. Baldwin and A. Lachlan, On strongly minimal sets, J. Symbolic Logic 36 1971, 79-96. MR 44:3851

[Ch] G. Cherlin, Groups of small Morley rank, Ann. of Math. Logic 17, 1979, 1-28. MR 81h:03072

[CK] C. C. Chang and H. J. Keisler, Model theory, North-Holland, Amsterdam, 1973. MR 53:12927

[EH] D. Evans and E. Hrushovksi, Projective planes in algebraically closed fields, Proc. London Math. Soc. (3) 62, 1991, 1-24. MR 92a:05031

[FJ] M. Fried and M. Jarden, Field arithmetic, Springer-Verlag, Berlin, 1986. MR 89b:12010

[GH] P. Griffiths and J. Harris, Principles of algebraic geometry, Wiley-Interscience, New York, 1978. MR 80b:14001

[Ha] R. Hartshorne, Algebraic geometry, Springer, New York, 1977. MR 57:3116

$[\mathrm{HH}]$ V. Harnik and L. Harrington, Fundamentals of forking, Ann. Pure Appl. Logic 26, (1984). MR 86c:03032

[HL] E. Hrushovski and J. Loveys, Locally modular strongly minimal sets, (to appear).

[HP] E. Hrushovski and A. Pillay, Weakly normal groups, Logic Colloquium' 85 (Paris), NorthAmsterdam, 1987. MR 88e:03051

[Hr1] E. Hrushovski, Unimodular minimal structures, J. London Math. Soc. (2) 46, (1992), 385396. MR 94b:03062

[Hr2] _ Locally modular regular types, Classification Theory (Chicago 1985) (J. T. Baldwin, ed.), Springer-Verlag, Berlin and New York, 1987. MR 90m:03064

[Hr3] _ A new strongly minimal set, Ann. Pure Appl. Logic 62, (1993), 147-166. MR 94d:03064

[Hr4] Strongly minimal expansions of algebraically closed fields, Israel J. Math. 79, (1992), 129-151. MR 95c:03078

[Hr5] _ Recognizing groups in homogeneous geometries, preprint.

[Hr6] Almost orthogonal regular types, Ann. Pure Appl. Logic 45, (1989), 139-155. MR 91k:03083

[Hr7] _Kueker's conjecture for stable theories, J. Symbolic Logic 54, (1989), 221-225. MR 90b:03046

[HZ] E. Hrushovski and B. Zilber, Zariski geometries, Bull. Amer. Math. Soc. (N.S.) 28, (1993), 315-323. MR 93j:14003

[L] S. Lang, Introduction to algebraic geometry, Interscience, New York, 1964. MR 20:7021

[Ma] A. Macintyre, On aleph-one categorical theories of fields, Fund. Math. 71, (1971), 1-25. MR 45:48

[Mo] M. Morley, Categoricity in power, Trans. Amer. Math. Soc. 114, (1965), 514-538. MR 31:58

[Pi1] A. Pillay, An introduction to stability theory, Oxford Univ. Press, London and New York, 1983. MR 85i:03104

[Pi2] Anand Pillay (forthcoming), 
[Po] Bruno Poizat, Missionary mathematics, J. Symbolic Logic 53, (1988), 137-145. MR 89b:03058

[W] A. Weil, On algebraic groups of transformations, Amer. J. Math. 77, (1955), 355-391. MR 17:533e

[Z] B. Zilber, The structure of models of uncountably categorical theories, Proc. Internat. Congr. Math. (Warsaw 1983), vol. 1, North-Holland, Amsterdam, 1984, pp. 359-368. MR 87d:03093b

Abstract. A characterization is obtained of the Zariski topology over an algebraically closed field.

Department of Mathematics, Hebrew University, Jerusalem, Israel

E-mail address: ehud@sunset.ma.huji.ac.il

Department of Mathematics, Kemerovo University, Kemerovo, Russia 